\title{
SALUD PASO POR PASO: A CULTURALLY-TAILORED PHYSICAL ACTIVITY \\ INTERVENTION WITH HISPANIC ADULTS WITH TYPE 2 DIABETES MELLITUS
}

A Dissertation
Presented to
the Faculty of the Graduate School
at the University of Missouri-Columbia
In Partial Fulfillment
of the Requirements for the Degree
Doctor of Philosophy
JULIO CESAR LOYA
Dr. Maithe Enriquez, Dissertation Supervisor
JUL 2021


The undersigned, appointed by the dean of the Graduate School, have examined the dissertation entitled

SALUD PASO POR PASO: A CULTURALLY-TAILORED PHYSICAL ACTIVITY INTERVENTION WITH HISPANIC ADULTS WITH TYPE 2 DIABETES MELLITUS presented by Julio Cesar Loya, a candidate for the degree of Doctor of Philosophy, and hereby certify that, in their opinion, it is worthy of acceptance.

Professor Maithe Enriquez

Professor Vicki Conn

Professor Bonnie Wakefield

Professor Stephanie Potochnick 


\section{DEDICATION}

There are two special persons without whom this journey would not have been possible. First and foremost, I would like to thank my wife, Krista Loya, for her unconditional love during this process. Without your patience, encouragement, and support, I could not have achieved this. I will never be able to repay you for all the missed vacations and late nights, but I will try for the rest of our lives. Thank you for being my love, best friend, and cheerleader.

I would also like to thank my late mother, Carmen Leon, for helping me become the person I am today. Your examples of hard work, perseverance, and dedication to excellence are lessons that will continue to push me forward. Thank you for being the best mom.

This dissertation is for both of you. 


\section{ACKNOWLEDGEMENTS}

Completion of a doctoral degree is a significant undertaking that requires the support of many people. I would like to thank my dissertation chair, Dr. Maithé Enriquez, for her incredible encouragement and support during this process. Thank you for encouraging many years ago to consider applying to the doctoral program at the Sinclair School of Nursing; it is one of the best decisions I have ever made. I would also like to thank Dr. Vicki Conn for being my original dissertation chair and continuing committee member. Your guidance and insights allowed me to focus my research interests. I also owe gratitude to Dr. Bonnie Wakefield and Dr. Stephanie Potochnick for their willingness to serve as my committee members, as well as Dr. Jo-Ana Chase and Dr. Lorraine Phillips for their willingness to serve as past committee members. A special thank you goes to Dr. Deidre Wipke-Tevis, for always looking for ways with which to enhance the support and experiences of the doctoral students. I would like to acknowledge the support of the faculty of the Sinclair School of Nursing at the University of Missouri for imparting their knowledge and wisdom so freely and enthusiastically. 


\section{TABLE OF CONTENTS}

ACKNOWLEDGEMENTS ........................................................ ii

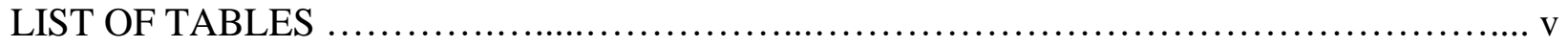

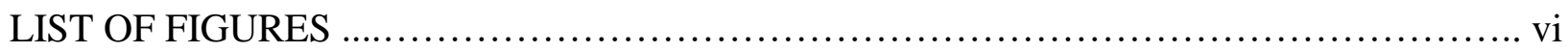

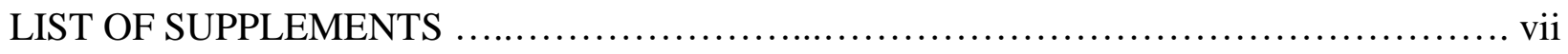

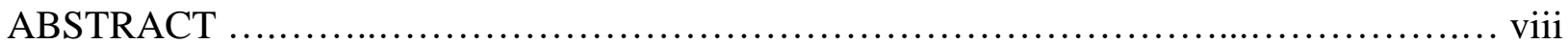

Chapter

1. INTRODUCTION: General introduction ..................................... 1

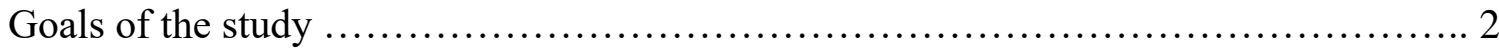

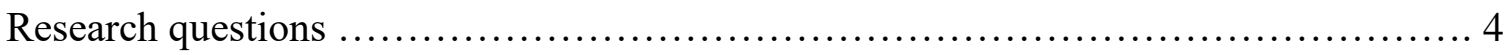

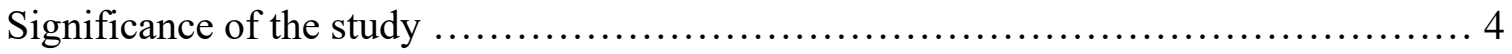

References ................................................................. 5

2. SYSTEMATIC REVIEW OF PHYSICAL ACTIVITY INTERVENTIONS AND

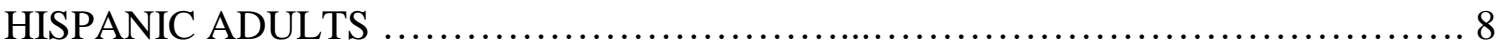

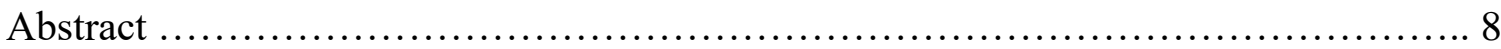

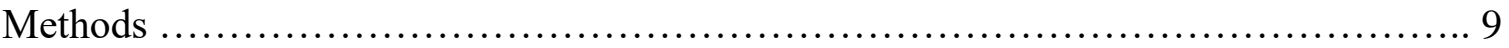

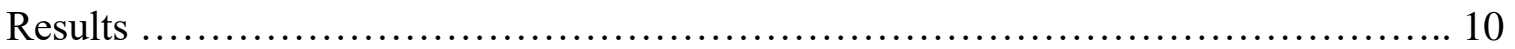

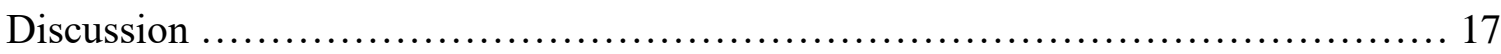

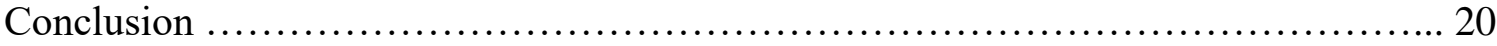

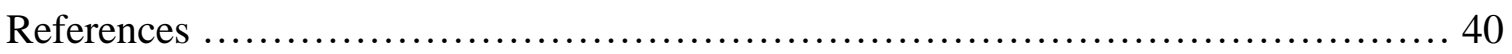

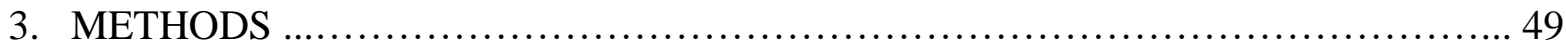

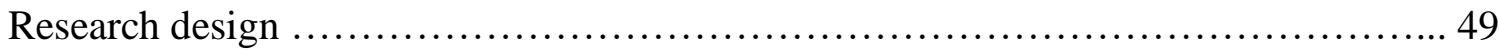


Sample 49

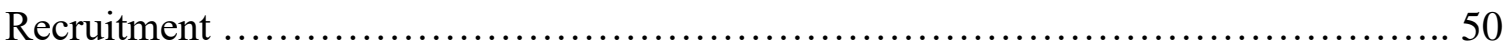

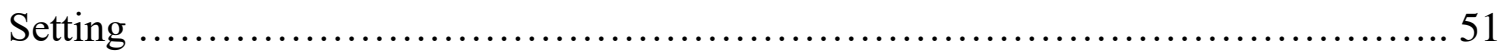

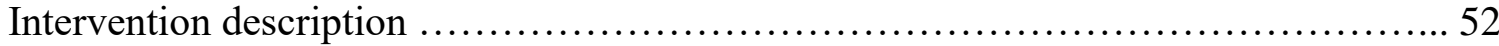

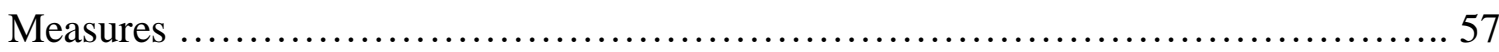

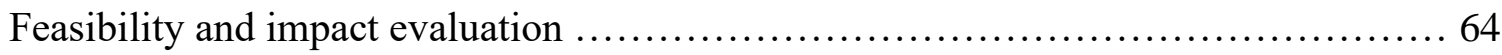

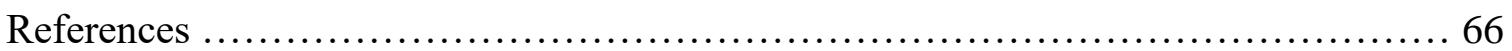

4. RESULTS: Salud Paso por Paso - A culturally-tailored physical activity intervention with Hispanic adults with type 2 diabetes mellitus ................................ 79

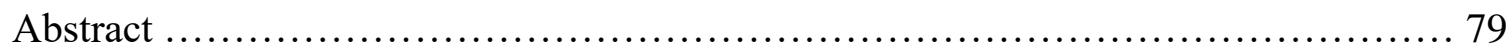

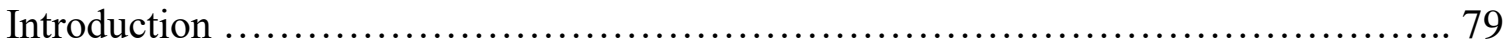

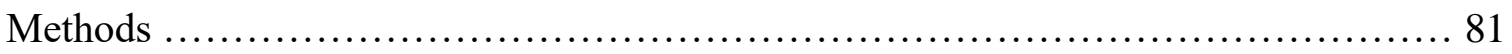

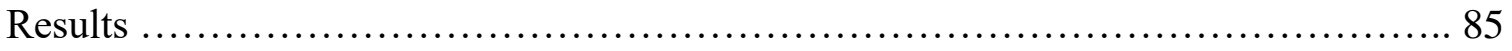

Discussion ........................................................................ 90

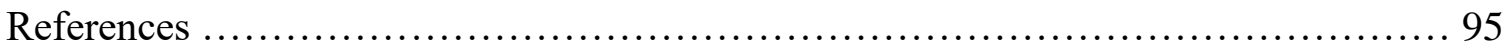

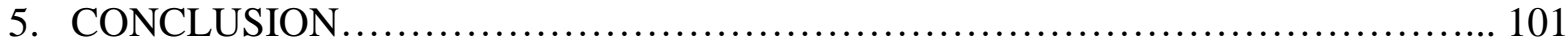

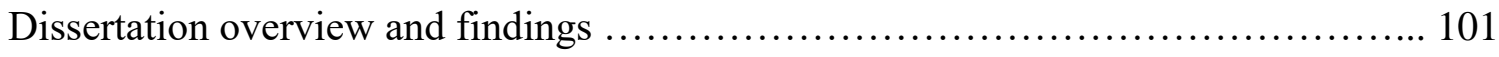

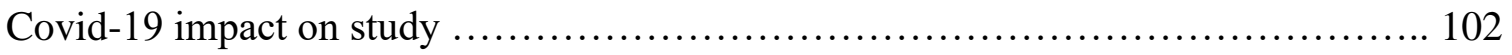

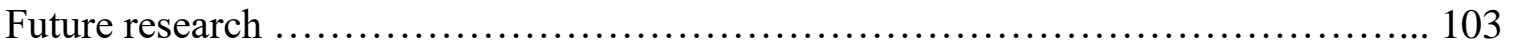

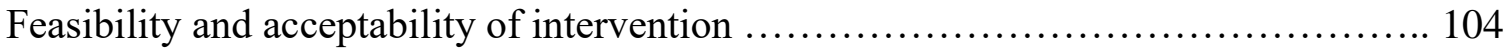

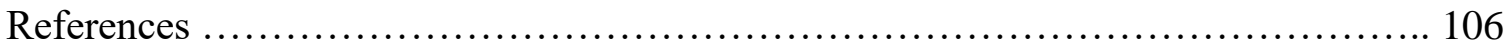

6. COMPREHENSIVE REFERENCE LIST ..................................... 108

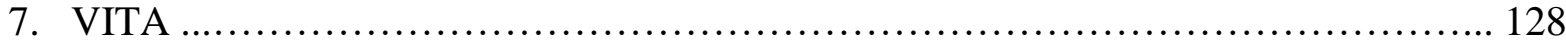




\section{LIST OF TABLES}

1. Randomized, Controlled PA Intervention Studies with Hispanic Adults ............... 23

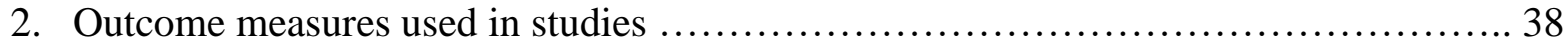

3. Intervention Activities ................................................. 54

4. Salud Paso por Paso Intervention Activities by Week ........................... 84

5. Salud Paso por Paso Participant Demographic and Health Characteristics .............. 86

6. Salud Paso por Paso Differences in Number of Steps and Minutes of Exercise by Week

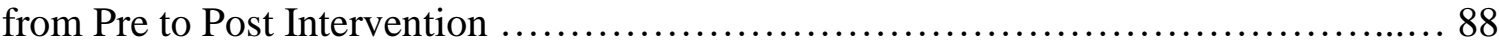

7. Salud Paso por Paso Relationships Between Demographic Characteristics \& Steps/

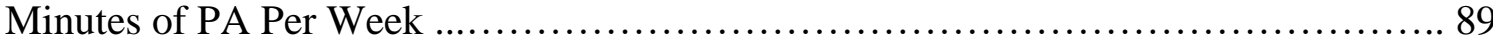




\section{LIST OF FIGURES}

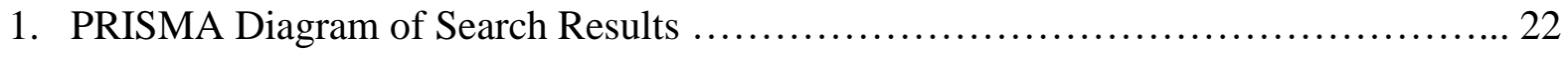




\section{LIST OF SUPPLEMENTS}

1. Study Flow Chart of Hispanic Adults in Physical Activity Intervention ................ 71

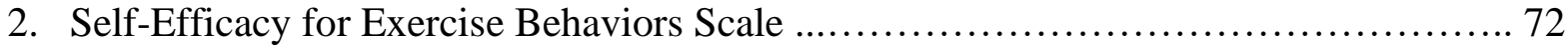

3. 7-Day Physical Activity Recall Questionnaire .............................. 74

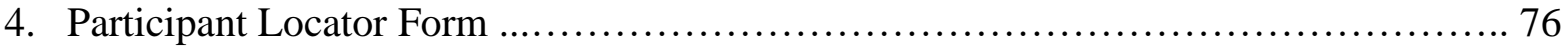

5. Field Notes Guide of Clinica Amistad Intervention ............................... 77

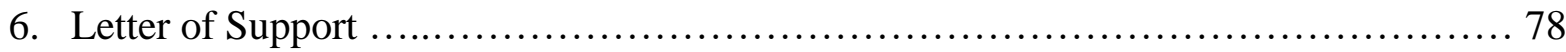


Salud Paso por Paso: A culturally-tailored physical activity intervention with Hispanic adults with type 2 diabetes mellitus

Julio Cesar Loya, BSN, RN

Dr. Maithé Enriquez, Dissertation Advisor

\begin{abstract}
Limited information is available regarding culturally-tailored physical activity (PA) interventions for Hispanic adults with type 2 diabetes mellitus (T2DM). A community-partnered approach was used to examine a novel culturally-tailored PA intervention using a pre-post, no control group design. The intervention consisted of six weekly 45 -minute sessions for participants to engage in PA led by the researcher. A total of 21 individuals participated in the study. The typical participant was a 53-year-old female (90\%) Hispanic adult living with T2DM with low acculturation. On average, before the intervention, the participants walked 10,285 (sd 14,779) steps per week with 43.4 (sd 68.1) minutes of PA per week. Despite implementation during the COVID-19 pandemic, the intervention was feasible and acceptable, and 19 (90.5\%) participants attended all intervention sessions. There were significant increases in steps per week $(p=0.007 ; d=1.03)$ and minutes of PA per week $(p=0.000 ; d=1.62)$. Findings suggest that Salud Paso por Paso has promise as a strategy to enhance PA behaviors in the priority population. A randomized, controlled trial with a larger study sample is warranted to examine efficacy and impact on the diabetes health outcomes of Hispanic adults with T2DM.
\end{abstract}




\section{CHAPTER 1}

\section{GENERAL INTRODUCTION}

Diabetes mellitus is a metabolic disease that affects approximately 34 million individuals in the United States (Centers for Disease Control and Prevention [CDC], 2019a). Furthermore, over 90\% these cases are classified as diabetes mellitus type 2 (T2DM) (CDC, 2019a).

Prediabetes is a related condition where blood glucose levels are higher than normal, but not yet reaching T2DM levels (CDCa, 2020). Approximately 88 million adults in the United States have prediabetes, with more than $84 \%$ not knowing they have it (CDC, 2020). Risk factors for T2DM include prediabetes, overweight, age 45 years or older, immediate family members with T2DM, physical activity (PA) less than 3 times per week, history of gestational diabetes, and being African American, Hispanic/Latino American, Native American, or Alaska Native (CDC, 2021b). The human body converts most of the food eaten into glucose, which is then released into the bloodstream for uptake by cells with the assistance of insulin (CDC, 2019a). T2DM is a chronic condition where the body does not produce enough insulin or cannot use the insulin available as efficiently as possible. Over time, the increase in blood levels of glucose, or hyperglycemia, has deleterious effects on various body systems. Complications of T2DM can develop into serious and life-threatening health issues, such as cardiovascular disease, renal disease, neuropathy, gastroparesis, sexual dysfunction, genitourinary issues, oral health issues, and vision and hearing loss (CDC, 2019b).

The Hispanic population in the United States is disproportionally affected by the burden of T2DM. Hispanic adults have a greater than $50 \%$ chance of developing diabetes, compared to a 40\% chance for U.S. adults (CDC, 2021a). Additionally, evidence shows that Hispanic individuals with T2DM have a higher incidence and prevalence of nephropathy, diabetic eye 
disease, and higher incidence of diabetes-related amputations (Aguayo-Mazzucato et al., 2019). Furthermore, evidence suggests older Hispanic adults incur higher costs for diabetes care than non-Hispanic whites, with more acute hospital admissions and longer lengths of stay (Glantz et al., 2019).

Due to the disproportionate burden the Hispanic population faces with T2DM, there is a palpable need to elucidate ways with which to ameliorate the deleterious effects of this chronic condition. This dissertation project was completed to determine the effectiveness of a culturallytailored PA intervention in Hispanic adults with T2DM.

\section{Current Research}

A review of the literature of physical activity (PA) interventions in Hispanic adults shows a variety of approaches to engage participants in PA (Loya, 2018). The majority of the studies in this literature review examined interventions based on walking. The review also identified a variety of theoretical frameworks and tools with which PA was measured. Studies with a theoretical framework showed significant increases in PA. Additionally, face-to-face delivery formats seem to enhance the effectiveness of interventions. Many of the studies included in this review did not appear to incorporate culturally appropriate interventions to target subsets of the Hispanic population.

\section{Purpose of the Study}

Using a pre-test/post-test, no control group design, this pilot study examined the feasibility, acceptability, and impact of a novel PA intervention among Hispanic adults (n=21); the name of the intervention is Salud Paso por Paso (Health Step by Step). Outcomes of PA measured included (1) total number of steps per week per participant, (2) total minutes of PA per week per participant, (3) 7-day PA recall, and (4) self-efficacy for PA scores. Demographic data 
collected included: age, gender, ethnicity, marital status, yearly household income, education level, years living in the United States, primary language at home, presence of health conditions in addition to diabetes, and baseline acculturation scores.

\section{Theoretical Framework}

The theoretical framework that guided this pilot intervention study was Social Cognitive Theory (SCT) by Albert Bandura. One of the main constructs of SCT is the concept of selfefficacy (Bandura, 1989, 2004). Self-efficacy serves as the foundation of behavior change to motivate individuals to engage in PA. Knowledge of health risks and benefits related to health practices is another construct of SCT (Bandura, 2004). This construct posits that individuals are likely to change their behavior if they are self-aware of how increases in PA positively affect health. The operationalization of self-efficacy and knowledge of health risks and benefits constructs occurred through the delivery of tailored content during face-to-face meetings with participants in the study.

\section{Acculturation}

A potential limitation in PA behavior change research with the Hispanic population is a tendency to group all individuals together that identify as Hispanic. Within the Hispanic designation, there are multiple subsets, and a few examples include: Mexican, Puerto Rican, Cuban, Guatemalan, Honduran, Colombian, and Venezuelan (Ennis, Ríos-Vargas, \& Albert, 2011). Acculturation is the process by which Hispanics are exposed to mainstream cultural patterns in the United States and subsequent modification in values, norms, attitudes, and behaviors as a result of this exposure (Marin et al., 1987). Evidence suggests that acculturation affects rates of obesity and overweight for Mexican Americans (Garcia et al., 2012), and least acculturated Hispanic individuals engage in more moderate-to-vigorous PA (Joseph et al., 2018). 
Additionally, there is evidence suggesting that Hispanic adults such as Mexican-Americans do not meet recommended levels of PA (Tucker, Welk, \& Beyler, 2011).

\section{Research Question}

The goal of this pilot study was to examine feasibility, acceptability, and impact of a culturally-tailored PA intervention for Hispanic adults with T2DM. Main outcome measures of interest to this study were changes in PA and self-efficacy. The study design used communitybased participatory research (CBPR) principles, including action-oriented community diagnosis (Eng \& Blanchard, 2007). The study addressed the following research questions:

1. Is the Salud Paso por Paso intervention feasible and acceptable in a group of adults living with T2DM and receiving care at Clinica Amistad?

2. What is the impact of the Salud Paso por Paso intervention, from pre- to postintervention on number of steps and minutes of PA?

Significance of the Study

The crucial significance of this study was to discern the effectiveness of a culturallytailored PA intervention with a sample of Hispanic adults using a theoretical framework and community-based participatory research (CBPR) principles. Rather than utilizing a prescriptive approach to engage in PA, participants in this study received tailored information and personalized encouragement. Utilizing CBPR principles in research requires a partnership that involves community members, practitioners, and academic researchers to address health concerns in a community setting (Israel et al., 2010). Moreover, findings from this pilot study will assist in informing a larger prospective, randomized controlled intervention study. This dissertation study is the first step in a program of research forward that aims to ameliorate the potential negative effects of T2DM, among Hispanics, through engagement in PA. 


\section{References}

Aguayo-Mazzucato, C., Diague, P., Hernandez, S., Rosas, S., Kostic, A., \& Caballero, A. E. (2019). Understanding the growing epidemic of type 2 diabetes in the Hispanic population living in the United States. Diabetes Metabolism Research and Reviews, 35(2), e3097. https://doi.org/10.1002/dmrr.3097

Bandura, A. (1989). Human agency in social cognitive theory. American Psychologist, 44(9), 1175-1184. https://doi.org/10.1037/0003-066X.44.9.1175

Bandura, A. (2004). Health promotion by social cognitive means. Health Educucation \& Behavior, 31(2), 143-164. https://doi.org/10.1177/1090198104263660

Centers for Disease Control and Prevention. (2019a, May 30). Diabetes basics: Type 2 diabetes. https://www.cdc.gov/diabetes/basics/type2.html

Centers for Disease Control and Prevention. (2019b, August 1). Living with diabetes - Prevent complications. https://www.cdc.gov/diabetes/managing/problems.html

Centers for Disease Control and Prevention. (2020, June 11). Diabetes basics: Prediabetes your chance to prevent type 2 diabetes. https://www.cdc.gov/diabetes/basics/prediabetes.html

Centers for Disease Control and Prevention. (2021a, April 7). Hispanic/Latino Americans and type 2 diabetes. https://www.cdc.gov/diabetes/library/features/hispanic-diabetes.html

Centers for Disease Control and Prevention. (2021b, April 23). Diabetes basics: Diabetes risk factors. https://www.cdc.gov/diabetes/basics/risk-factors.html

Eng, E., \& Blanchard, L. (2007). Action-oriented community diagnosis: A health education tool. International Quarterly of Community Health Education, 26(2), 141-158. https://doi.org/10.2190/8046-2641-7HN3-5637 
Ennis, S. R., Ríos-Vargas, M., \& Albert, N. G. (2011). The Hispanic population: 2010. https://www.census.gov/prod/cen2010/briefs/c2010br-04.pdf

Garcia, L., Gold, E. B., Wang, L., Yang, X., Mao, M., \& Schwartz, A. V. (2012). The relation of acculturation to overweight, obesity, pre-diabetes and diabetes among U.S. MexicanAmerican women and men. Ethnicity \& Disease, 22(1), 58-64.

Glantz, N. M., Duncan, I., Ahmed, T., Fan, L., Reed, B. L., Kalirai, S., \& Kerr, D. (2019). Racial and ethnic disparities in the burden and cost of diabetes for US Medicare beneficiaries. Health Equity, 3(1), 211-218. https://doi.org/10.1089/heq.2019.0004

Israel, B. A., Coombe, C. M., Cheezum, R. R., Schulz, A. J., McGranaghan, R. J., Lichtenstein, R., Reyes, A. G., Clement, J., \& Burris, A. (2010). Community-based participatory research: A capacity-building approach for policy advocacy aimed at eliminating health disparities. American Journal of Public Health, 100(11), 2094-2102. https://doi.org/10.2105/AJPH.2009.170506

Joseph, R. P., Benitez, T. J., Ainsworth, B. E., Todd, M., \& Keller, C. (2018). Acculturation and physical activity among Latinas enrolled in a 12-month walking intervention. Western Journal of Nursing Research, 40(7), 942-960. https://doi.org/10.1177/0193945917692305

Loya, J. C. (2018). Systematic review of physical activity interventions in Hispanic adults. Hispanic Health Care International, 16(4), 174-188. https://doi.org/10.1177/1540415318809427

Marin, G., Sabogal, F., Marin, B. V., Otero-Sabogal, R., \& Perez-Stable, E. J. (1987). Development of a short acculturation scale for Hispanics. Hispanic Journal of Behavioral Sciences, 9(2), 183-205. 
Tucker, J. M., Welk, G. J., \& Beyler, N. K. (2011). Physical activity in U.S.: Adults compliance with the Physical Activity Guidelines for Americans. American Journal of Preventive Medicine, 40(4), 454-461. https://doi.org/10.1016/j.amepre.2010.12.016 
CHAPTER 2

\title{
SYSTEMATIC REVIEW OF PHYSICAL ACTIVITY INTERVENTIONS AND HISPANIC ADULTS
}

Loya, J. C. (2018). Systematic review of physical activity interventions in Hispanic adults. Hispanic Health Care International, 16(4), 174-188. https://doi.org/10.1177/1540415318809427

\begin{abstract}
Abstract: Introduction: Physical activity (PA) has demonstrated substantial physical and psychological benefits. However, Hispanics engage in less leisure-time PA when compared to other groups, putting them at higher risk for diseases associated with obesity, such as diabetes mellitus type 2 . This literature review was conducted to identify best practices with regard to interventions designed to increase PA among Hispanic adults. Methods: Extensive searching located 21 randomized, controlled studies conducted in the United States. Results: Common conceptual frameworks were the transtheoretical model and social cognitive theory. Most interventions used educational sessions with a variety of topics and many used promotoras to increase PA. Outcomes were predominantly examined using self-report PA measures. Walking was the most commonly reported PA behavior. Studies with significant results were those that measured moderate-to-vigorous PA and used theory to guide interventions. Male and older participants were underrepresented. Conclusion: Effective culturally appropriate PA interventions for Hispanics adults are needed. Particular attention to intervention tailoring based on country of origin could enhance intervention effectiveness. Keywords: physical activity, Latino, adults, systematic review
\end{abstract}




\section{Introduction}

The link between physical inactivity and overweight/obesity has been well documented (Kwon, Wang, \& Hawkins, 2016). Overweight/obesity is associated with the development of chronic conditions such as diabetes mellitus type 2 , coronary heart disease, and hypertension (Centers for Disease Control and Prevention [CDC], 2018). Approximately 69\% of adults are overweight/obese (National Institute of Diabetes and Digestive and Kidney Diseases, 2017) and only 1 in 5 adults meets the recommendations of at least 150 minutes of physical activity (PA) every week (CDC, 2014). Hispanics, the fastest growing group in the United States (US), engage in less leisure-time PA than other subsets of the US population and are more likely to be diagnosed with diabetes mellitus type 2 (Brown, 2014; Marquez, Neighbors, \& Bustamante, 2010).

Due to the burden that chronic health conditions place on Hispanic adults, it is critical to promote PA in this population. Previous published reviews focusing on factors that promote PA have been limited to healthy adults (Conn, Phillips, Ruppar, \& Chase, 2012), females (Keller \& Fleury, 2006; Perez, Fleury, \& Keller, 2010; Sharma, 2008), non-Hispanic samples or to acculturation without a PA emphasis (Ickes \& Sharma, 2012; Martinez, Ainsworth, \& Elder, 2008; Mier, Ory, \& Medina, 2010). This literature review aimed to identify intervention strategies to increase PA that are culturally appropriate, and effective, for US Hispanic adults. Such strategies could be used in clinical practice to enhance the health of this population.

\section{Methods}

Inclusion criteria for the literature search were randomized, controlled PA intervention studies that included at least $75 \%$ US Hispanic adults (i.e. age 18 or older) as participants. PubMed, the Cumulative Index to Nursing and Allied Health Literature (CINAHL), and 
PsycINFO were searched from start date to June 2018. Only randomized controlled trials, due to the stronger level of evidence, were included. Studies that did not include a measurable PA outcome measures were excluded as were family centered interventions that focused on PA behaviors of children.

\section{Search Strategy}

Searches were conducted following PRISMA guidelines (Moher, Liberati, Tetzlaff, Altman, \& the PRISMA Group, 2009) and in the same manner for each database using the terms "physical activity," "intervention," "Hispanic," "Latino (a)" and "adults." For PubMed, the initial search yielded 882 results using the terms [physical activity AND intervention AND adult AND "Hispanic or Latino or Latina"]. After reading the titles and abstracts, 838 were excluded and 44 were read in full text. For CINAHL, 3,877 results were obtained; four were duplicates, 3853 were excluded based on the title and abstract, and 20 were read in full text. For PsycINFO there were 496 results, six duplicates, 481 were excluded based on the title/abstract and 9 studies read in full text. (Figure 1). An additional 15 articles were excluded because there was no intervention, 20 were not RCTs, 5 did not measure a PA outcome, and 12 focused on children. In the end, 21 studies were included in the review.

\section{Data extraction}

One author independently extracted data and synthesized the results. Demographic data (i.e. sample size, age, gender, ethnicity, country of origin, and health status), theoretical approach, intervention description, and research design was extracted for each study. In addition, the type of PA behavior and how it was measured as well as the pertinent results of each study were extracted.

\section{$\underline{\text { Results }}$}


The 21 studies (Table 1) ranged in dates of publication from January 2001 to June 2018. All studies were RCTs, however there was variation with regard to the intervention strategies and outcome measures utilized.

\section{Participant Characteristics}

Almost half (48\%) of the studies reported participant country of origin (Coleman et al., 2012; Hovell et al., 2008; Khare, Cursio, Locklin, Bates, \& Loo, 2014; King, Bickmore, Campero, Pruitt, \& Yin, 2013; Koniak-Griffin et al., 2015; Marcus et al., 2013; Marcus et al., 2016; Marshall et al., 2013; Pekmezi et al., 2009; Rosal et al., 2011) or self-identified ancestry and 16 studies referred to participants as Hispanic/Latino (Arredondo et al., 2017; Babamoto et al., 2009; Coleman et al., 2012; Duggan et al., 2014; Hawkins et al., 2015; Hovell et al., 2008; Khare et al., 2014; Koniak-Griffin et al., 2015; Marshall et al., 2013; Mitchell, Andrews, \& Schenker, 2015; Pekmezi et al., 2009; Poston et al., 2001; Rosal et al., 2011; Rosal et al., 2005; Rothschild et al., 2014; Vincent, 2009). Almost half (48\%) of studies had at least some participants that self-identified as Mexican or Mexican-American (Arredondo et al., 2017; Coleman et al., 2012; Hovell et al., 2008; Koniak-Griffin et al., 2015; Marcus et al., 2016; Marshall et al., 2013; McEwen, Pasvogel, Murdaugh, \& Hepworth, 2017; Poston et al., 2001; Rothschild et al., 2014; Vincent, 2009). Other countries of origin represented in the study samples were Mexico, Puerto Rico, Dominican Republic, Guatemala and Colombia (KoniakGriffin et al., 2015; Marcus et al., 2013; Marcus et al., 2016; Pekmezi et al., 2009; Rosal et al., 2011). All but two studies (Eakin et al., 2007; King et al., 2013) had study samples that were $100 \%$ Hispanic.

All studies had a majority of female participants, with 11 studies reporting $100 \%$ female participants (Arredondo et al., 2017; Coleman et al., 2012; Hawkins et al., 2015; Hovell et al., 
2008; Khare et al., 2014; Koniak-Griffin et al., 2015; Marcus et al., 2013; Marcus et al., 2016;

Marshall et al., 2013; Pekmezi et al., 2009; Poston et al., 2001). Sample sizes varied from 20 to 1093 participants and only two studies had participants that were over 64 years of age (King et al., 2013; McEwen et al., 2017). Most participants that were between the ages of 18 and 64 and study samples were relatively homogenous. Men and older adults were underrepresented, with only half (48\%) of the studies enrolling male participants (Babamoto et al., 2009; Duggan et al., 2014; Eakin et al., 2007; King et al., 2013; McEwen et al., 2017; Mitchell et al., 2015; Rosal et al., 2011; Rosal et al., 2005; Rothschild et al., 2014; Vincent, 2009). No study had greater than $36 \%$ male participants.

Most of the studies (19 of 21) reported the health status of participants through measures such as diagnosed medical conditions or risk factors (e.g. elevated body mass index [BMI] or hypertension [HTN]). Two studies did not provide health status information (Khare et al., 2014; Marcus et al., 2016). Health indicators reported were BMI (Hovell et al., 2008; Marcus et al., 2013; Marshall et al., 2013; McEwen et al., 2017; Mitchell et al., 2015; Pekmezi et al., 2009), diabetes mellitus (Arredondo et al., 2017; Duggan et al., 2014; Koniak-Griffin et al., 2015; Rosal et al., 2011; Rosal et al., 2005; Rothschild et al., 2014; Vincent, 2009), multiple health conditions (Arredondo et al., 2017; Eakin et al., 2007; King et al., 2013), gestational diabetes (Hawkins et al., 2015), and hyperlipidemia (Babamoto et al., 2009; Coleman et al., 2012).

\section{Duration of studies}

The follow-up period ranged from 3 to 24 months; however, most (62\%) studies had follow-up periods between 3 and 6 months (Babamoto et al., 2009; Coleman et al., 2012; Duggan et al., 2014; Eakin et al., 2007; King et al., 2013; Marcus et al., 2013; Marcus et al., 2016; Marshall et al., 2013; McEwen et al., 2017; Mitchell et al., 2015; Pekmezi et al., 2009; 
Rosal et al., 2005; Vincent, 2009). One study followed participants for 6 weeks post-partum (Hawkins et al., 2014). One study had a follow-up period of 9 months (Koniak-Griffin et al., 2014). Five studies had follow-up periods of 12 months (Arredondo et al., 2017; Hovell et al., 2008; Khare et al., 2014; Poston et al., 2001; Rosal et al., 2011). Only one study (Rothschild et al., 2014) followed participants for 12 months or longer.

\section{Measures}

Studies in this review used a wide variety of PA measures (Table 2). Some studies measured only the amount of activity (e.g. minutes, hours, days of PA), while others measured the amount and type of activity (e.g. mild, moderate, or vigorous). There were studies that measured kilocalories or metabolic equivalent minutes, while others did not measure these variables.

\section{Interventions}

\section{Theoretical Frameworks}

Sixteen studies (Arredondo et al., 2017; Coleman et al., 2012; Eakin et al., 2007; Hawkins et al., 2015; Hovell et al., 2008; Khare et al., 2014; King et al., 2013; Marcus et al., 2013; Marcus et al., 2016; Marshall et al., 2013; Pekmezi et al., 2009; Poston et al., 2001; Rosal et al., 2011; Rosal et al., 2005; Rothschild et al., 2014; Vincent, 2009) reported a guiding framework and six interventions were based in transtheoretical model of change (TTM) and social cognitive theory (SCT) (Hawkins et al., 2015; Khare et al., 2014; King et al., 2013; Marcus et al., 2013; Marcus et al., 2016; Pekmezi et al., 2009). Guiding theories included SCT (Poston et al., 2001; Rosal et al., 2011; Rosal et al., 2005; Vincent, 2009), the socio-ecological model (Arredondo et al., 2017; Eakin et al., 2007; Marshall et al., 2013), behavior change theory (Coleman et al., 2012), operant learning theory and applied behavior analysis (Hovell et al., 
2008), and self-management theory (Rothschild et al., 2014). Nine studies that mentioned a theoretical framework had statistically significant results (Arredondo et al., 2017; Coleman et al., 2012; Hawkins et al., 2015; Hovell et al., 2008; King et al., 2013; Marcus et al., 2013; Marcus et al., 2016; Poston et al., 2001; Vincent, 2009). Of those with significant results, six used either SCT or a combination of SCT and the TTM (Hawkins et al., 2015; King et al., 2013; Marcus et al., 2013; Marcus et al., 2016; Poston et al., 2001; Vincent, 2009). Other studies with statistically significant results used behavior change theory (Coleman et al., 2012), ecological framework (Arredondo et al., 2017), and operant learning theory (Hovell et al., 2008).

\section{Delivery of Interventions}

There were different modalities of intervention delivery described in the studies. Nine studies used community health workers or promotoras (i.e. lay health advisors [Martinez, Arredondo, \& Roesch, 2013]) to engage participants in the interventions (Arredondo et al., 2017; Babamoto et al., 2009; Coleman et al., 2012; Duggan et al., 2014; Koniak-Griffin et al., 2015; Marshall et al., 2013; McEwen et al., 2017; Mitchell et al., 2015; Vincent, 2009). Promotoras not only facilitated intervention sessions, they typically participated by engaging in PA behaviors themselves. Studies that used promotoras (Arredondo et al., 2017; Babamoto et al., 2009; Koniak-Griffin et al., 2015; Marshall et al., 2013; McEwen et al., 2017; Mitchell et al., 2015; Vincent, 2009) delivered face-to-face educational sessions at a predetermined location or at the participant's home. The rest of the studies used research staff (i.e. professionals) to deliver interventions (Coleman et al., 2012; Duggan et al., 2014; Eakin et al., 2007; Hawkins et al., 2015; Hovell et al., 2008; Khare et al., 2014; King et al., 2013; Marcus et al., 2013; Marcus et al., 2016; Pekmezi et al., 2009; Poston et al., 2001; Rosal et al., 2011; Rosal et al., 2005; Rothschild et al., 2014). Most studies used face-to-face educational sessions (Arredondo et al., 
2017; Babamoto et al., 2009; Coleman et al., 2012; Duggan et al., 2014; Eakin et al., 2007;

Hawkins et al., 2015; Hovell et al., 2008; Khare et al., 2014; Koniak-Griffin et al., 2015;

Marshall et al., 2013; McEwen et al., 2017; Mitchell et al., 2015; Poston et al., 2001; Rosal et al., 2011; Rosal et al., 2005; Rothschild et al., 2014; Vincent, 2009) and nine of these had significant results (Arredondo et al., 2017; Babamoto et al., 2009; Coleman et al., 2012; Hawkins et al., 2015; Hovell et al., 2008; Koniak-Griffin et al., 2015; Mitchell et al., 2015; Poston et al., 2001; Vincent, 2009). Three studies provided print materials via mail to engage participants in PA behaviors (Arredondo et al., 2017; Marcus et al., 2013; Pekmezi et al., 2009), one study utilized a computer to deliver the PA intervention (King et al., 2013), and one study emailed materials to participants (Marcus et al., 2016). Of five studies that used non-face-to-face contact, four had significant results (Arredondo et al., 2017; King et al., 2013; Marcus et al., 2013; Marcus et al., 2016).

\section{Intervention Content and Dose}

Interventions varied with regard to the use of strategies to enhance, and measure, physical activity. Eight studies provided pedometers to the participants as well as activity logs to track PA (Hawkins et al., 2015; King et al., 2013; Koniak-Griffin et al., 2015; Marcus et al., 2013; Marshall et al., 2013; Pekmezi et al., 2009; Rosal et al., 2011; Vincent, 2009); five had significant results (Hawkins et al., 2015; King et al., 2013; Koniak-Griffin et al., 2015; Marcus et al., 2013; Vincent, 2009). Three studies provided accelerometers and had significant results (Arredondo et al., 2017; Koniak-Griffin et al., 2015; Marcus et al., 2016). Studies that provided pedometers to participants also provided logs to record PA. Hence, participants were aware of the measurement of PA by pedometer and also of the timeframe of data collection. 
One study utilized a virtual advisor with an embodied conversational agent on a computer at a community senior center (King et al., 2013). One study designed an aerobic intervention with a Latina aerobics instructor, which included dance sessions accompanied by salsa/merengue music (Hovell et al., 2008). Two studies used print materials that were personalized to the participant's current state of motivational readiness for PA and tip sheets on selected topics such as stretching or measuring heart rate (Marcus et al., 2013), and mailings of PA manuals matched to the participants' current level of motivational readiness (Pekmezi et al., 2009).

Twelve $(57 \%)$ of studies reported significant improvement in PA from pre-to post intervention. Eight of 10 studies that listed walking as the primary intervention for PA had significant results (Arredondo et al., 2017; Coleman et al., 2012; Hovell et al., 2008; King et al., 2013; Marcus et al., 2013; Marcus et al., 2016; Marshall et al., 2013; Vincent, 2009). Also, one study featured a combination of dancing and walking (Vincent, 2009) and dancing alone (Hovell et al., 2008) as the primary modes of PA. The study that had dancing alone as the PA behavior also had statistically significant results with more participants engaging in the PA behavior post intervention (Hovell et al., 2008). The study that had a combination of dancing and walking also had statistically significant results (Vincent, 2009).

The educational sessions covered PA topics such as walking, leisure-time activity, and self-management of diabetes (Arredondo et al., 2017; Babamoto et al., 2009; Coleman et al., 2012; Duggan et al., 2014; Hawkins et al., 2015; Hovell et al., 2008; Koniak-Griffin et al., 2015; McEwen et al., 2017; Rosal et al., 2011; Rosal et al., 2005; Vincent, 2009). Intervention sessions ranged in duration from 50 minutes (Coleman et al., 2012) to 2 hours (Koniak-Griffin et al., 2015). One study had only three intervention sessions delivered at 1 month, 2 months, and 6 
months, with significant results (Coleman et al., 2012). Finally, one study provided biweekly or monthly educational materials (Pekmezi et al., 2009), but did not have significant findings.

\section{Cultural Relevance of Interventions}

Many $(81 \%)$ of the studies mentioned cultural appropriateness as part of intervention development. Furthermore, $67 \%$ of the studies described cultural appropriateness explicitly (Babamoto et al., 2009; Hawkins et al., 2015; Hovell et al., 2008; King et al., 2013; KoniakGriffin et al., 2015; Marcus et al., 2013; Marshall et al., 2013; McEwen et al., 2017; Mitchell et al., 2015; Pekmezi et al., 2009; Poston et al., 2001; Rosal et al., 2011; Rosal et al., 2005; Vincent, 2009). Nine (43\%) studies provided detail on how interventions were adapted to be culturally appropriate (Hawkins et al., 2015; Hovell et al., 2008; Marshall et al., 2013; McEwen et al., 2017; Pekmezi et al., 2009; Poston et al., 2001; Rosal et al., 2011; Rosal et al., 2005; Vincent, 2009). Four (19\%) studies mentioned interventions as being culturally appropriate but did not provide specific cultural components (Babamoto et al., 2009; Eakin et al., 2007; Marcus et al., 2016; Rothschild et al., 2014). Four studies (19\%) provided succinct explanations regarding culturally appropriate interventions (King et al., 2013; Koniak-Griffin et al., 2015; Marcus et al., 2013; Mitchell et al., 2015). Finally, four (19\%) studies did not mention culturally appropriate component (Arredondo et al., 2017; Coleman et al., 2012; Duggan et al., 2014;

Khare et al., 2014).

\section{Discussion}

Engaging in leisure-time PA at least one hour per day decreases the probability of obesity (Sarma, Devlin, Gilliland, Campbell, \& Zaric, 2015) and reduces the risk for chronic health conditions, such as diabetes mellitus (Bell et al., 2014). This literature review identified 
effective strategies to increase PA among Hispanic adults. However, the studies in this review had mixed samples of Hispanics.

It is important to note that there may be differences between Hispanic adults related to country of origin. Evidence suggests that nearly 7 in 10 Hispanic adults do not believe that there is a shared culture among Hispanic populations in the United States (Taylor, Lopez, Martinez, \& Velasco, 2012). Therefore, preferences of Hispanic participants may differ with regard to tailoring of PA interventions. For example, this review found that studies that incorporated dancing and music had significant findings. However, musical styles vary among Hispanic cultures; the music preferences of Mexicans may differ from those of Central Americans, for example (Turino, 2003). Hence, selecting culturally appropriate music that caters to the musical taste of the participants' country of origin or ancestry may be an important factor in efforts to increase participation PA behaviors among Hispanics.

Also, Hispanic populations experience disparities in the incidence of type 2 diabetes mellitus, when compared to other US populations (Bullard et al., 2018). Effective PA interventions can address health disparities and reduce the incidence of obesity and diseases linked to inactivity, such as diabetes and cardiovascular disease.

The studies in this review had mixed samples of Hispanics. Mexican or MexicanAmerican ancestry was self-reported by participants in 10 studies, however the other 11 studies reported various countries of origin.

There were five studies (Hawkins et al., 2015; King et al., 2013; Pekmezi et al., 2009; Rosal et al., 2005; Vincent, 2009) with sample sizes of less than 100 participants, whicm may have limited the ability to detect intervention impact and differences between groups. Recruitment challenges or limited fiscal resources may have contributed to frequent small 
samples. Restricted age range may limit the generalizability of findings of this review. Most study participants were between 30 and 50 years of age, hence there was a lack of information about the impact of PA interventions among younger and older Hispanic adults.

The majority of the studies examined interventions based on walking. There was great variety in the way that PA was measured, with some studies focusing solely on the amount (e.g. number of minutes) of PA while other studies focused on the amount and type of PA (e.g. mild vs. vigorous). Two studies suggested that individuals of Mexican-American ancestry prefer walking to engage in PA (Mier, Medina, \& Ory, 2007; Wood, 2002). While walking appeared to be a convenient and inexpensive PA strategy, future research should explore other forms of more vigorous PA, such as dancing, which also appeared to be of interest to this subgroup.

Promotoras or lay health advisors were often used to promote the interventions among Hispanic populations. The use of promotoras may have had a dual purpose: to increase trust with and enhance cultural appropriateness of the interventions. Additionally, these lay health advisors were often bilingual members of the community who could readily communicate with Spanishspeaking participants. The ability to communicate in the participants' preferred language may facilitate PA behavior change. Findings suggests that the use of promotoras may influence the acceptance of interventions by Hispanic adults. However, more rigorous investigation comparing promotoras to other interventionists has been recommended to provide empirical evidence of promotora effectiveness (Ayala, Vaz, Earp, Elder, \& Cherrington, 2010; Rhodes, Foley, Zometa, \& Bloom, 2007).

Theoretically driven studies showed significant increases in PA, ranging from two- to ten-fold increase per week. The predominant theoretical frameworks used, SCT and TTM, focus 
on the capability of individuals to enact desired PA behaviors. Hence, these theories may be an appropriate foundation to develop PA interventions for Hispanic populations in the future.

The use of pedometers to measure outcomes may have enhanced PA in and of itself. Evidence for use of pedometers to promote walking has had mixed results in the literature (Ogilvie et al., 2007). However, the use of a pedometer seemed to provide immediate visual feedback about the quantity of steps (Gabrys, Sperzel, Bernhoerster, Banzer, \& Vogt, 2017), which may have motivated some individuals to engage in and sustain PA behaviors. Moreover, the relative cost of pedometers is low (Kolt et al., 2012; Sangster, Church, Haas, Furber, \& Bauman, 2015), which facilitates access to this tool.

There were limitations to this literature review. Some studies that are relevant to this review may have been missed due to inadequate indexing in available databases. Additionally, ancestry searches conducted by hand did not yield any studies; furthermore, no dissertations were included in this review nor were articles published in Spanish. Some studies included in this review had small sample sizes and many of the studies relied on self-report of PA by participants. While there is evidence to suggest that self-reported PA is reliable (Craig et al., 2003), there is also evidence confirming that individuals tend to underreport sedentary behavior and overestimate both intensity of PA and duration of moderate-to-vigorous PA (Cerin et al., 2016). Despite the heterogeneity among the US Hispanic adult population, several studies did not describe country of origin or ancestry. Additionally, all studies had primarily female participants.

\section{Conclusion}

This literature review described randomized, controlled studies that tested PA interventions in Hispanic samples in the United States. A number of effective strategies to 
enhance PA among Hispanic adults were identified. The use of promotoras or community health workers (CHWs), a theoretical framework, and face-to-face delivery formats appeared to enhance intervention effectiveness. The use of pedometers produced significant results in some studies, as did studies that measured moderate-to-vigorous PA. Innovative strategies, including a virtual advisor and tailored mailings, were also effective and served to enhance engagement in PA behaviors. However, there was wide variety with regard to the type of intervention implemented and the manner in which PA was measured.

Recommendations for future research include increased efforts to recruit male, younger and older participants. The use of more objective PA measures, rather than self-report, should be considered in future research. More consistency in the measures used to examine PA could enhance the ability to compare and contrast intervention effectiveness between studies. In addition, developing culturally appropriate interventions that target subsets of the Hispanic population appears to be missing from the literature. Interventions that are specifically tailored based on the participants' country of origin or ancestry may enhance the effectiveness of PA interventions. Tailoring may also help to sustain PA within the target populations.

Hispanics are the largest minority population in the United States. This population experiences increased rates of chronic health conditions, such diabetes mellitus type 2 and cardiovascular disease, which have been correlated to sedentary lifestyle behaviors and overweight/obesity (Caballero, 2005). Therefore, more research is warranted to develop effective intervention strategies that can increase PA in the growing population of US Hispanic adults. Further, such interventions must be tailored and amenable to implementation among America's diverse Hispanic communities. 

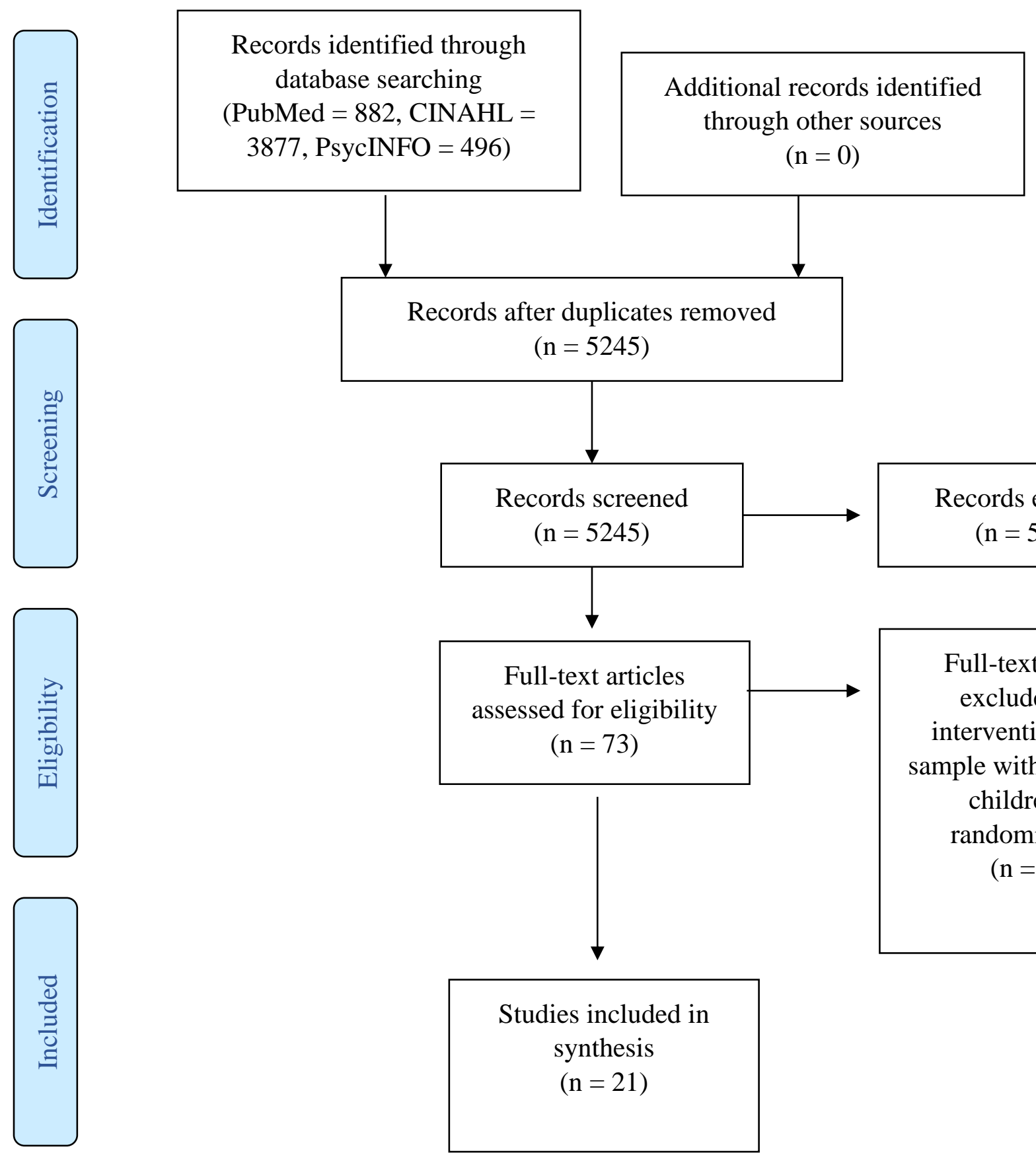

$$
(\mathrm{n}=0)
$$
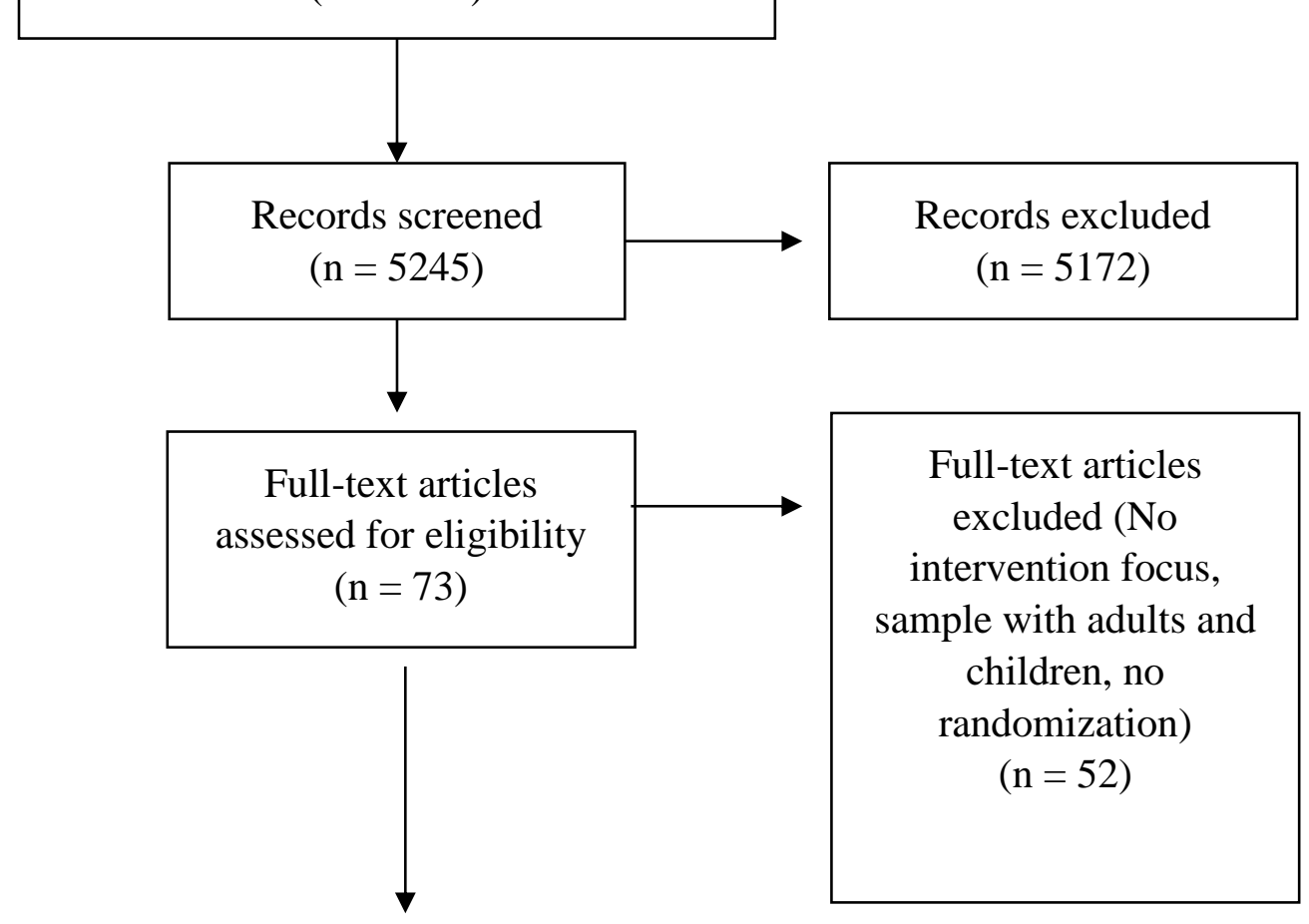

Studies included in synthesis

$$
(\mathrm{n}=21)
$$

Figure 2.1. PRISMA diagram of search results. Adapted from Moher, D., Liberati, A., Tetzlaff, J., Altman, D. G., The PRISMA Group (2009). Preferred Reporting Items for Systematic Reviews and MetaAnalyses: The PRISMA Statement. PLoS Med 6(7): e1000097. doi:10.1371/journal.pmed1000097 
Table 2.1

Randomized, controlled PA intervention studies with Hispanic adults ( $n=21)$

\begin{tabular}{|c|c|c|c|c|c|}
\hline $\begin{array}{l}\text { Author } \\
\text { Sample }\end{array}$ & $\begin{array}{l}\text { Theoretical } \\
\text { framework }\end{array}$ & Intervention & $\begin{array}{l}\text { Design } \\
\text { Follow-up } \\
\text { length }\end{array}$ & PA Measure & Results \\
\hline $\begin{array}{l}\text { Arredondo et al., } \\
2017 \\
\text { N= } 380 \\
\text { Females, mean } \\
\text { age (SD) } 44.4, \\
44.4 \\
\text { (100\% Latina), } \\
\text { various } \\
\text { participants with } \\
\text { T2DM, arthritis, } \\
\text { heart disease, } \\
\text { cancer. }\end{array}$ & $\begin{array}{l}\text { Ecological } \\
\text { framework } \\
\text { Promotora } \\
\text { model }\end{array}$ & $\begin{array}{l}\text { Weekly promotora-led free } \\
\text { PA classes } \\
\text { Start group with prayer, end } \\
\text { class with review of monthly } \\
\text { mailed health handouts with } \\
\text { topics including setting goals } \\
\text { and overcoming barriers to } \\
\text { PA and short discussion on } \\
\text { how to apply lesson or skill } \\
\text { Culturally appropriate } \\
\text { component: Not specified }\end{array}$ & $\begin{array}{l}\text { Cluster } \\
\text { randomized } \\
\text { controlled trial } \\
(2 \text { walking } \\
\text { groups, 2 } \\
\text { cardio dance } \\
\text { classes, and } 2 \\
\text { strength } \\
\text { training } \\
\text { classes }) \\
\text { 12-months }\end{array}$ & $\begin{array}{l}\text { PA accelerometer } \\
\text { weekly averages of } \\
\text { MVPA } \\
\text { Self-report PA } \\
\text { World Health } \\
\text { Organization } \\
\text { Global Physical } \\
\text { Activity } \\
\text { Questionnaire: } \\
\text { minutes per week } \\
\text { of leisure time } \\
\text { MVPA }\end{array}$ & $\begin{array}{l}\text { Accelerometer-based MVPA: } \\
\text { Measured in logged units } \\
\text { PA adjusted mean (SE) } \\
\text { Intervention group }(\mathrm{IG})=4.93(0.05) \\
\frac{\text { Control group }(\mathrm{CG})=4.78(0.03)}{\text { Difference in adjusted means } 0.15, p=0.03} \\
\text { Self-report leisure time PA: } \\
\text { Measured in logged units } \\
\text { PA adjusted mean (SE) } \\
\frac{\mathrm{IG}}{\mathrm{CG}}=4.86(0.05) \\
\text { Difference in adjusted means } 0.39, p=0.003 \\
\text { IG participants had significant results }\end{array}$ \\
\hline $\begin{array}{l}\text { Babamoto et al., } \\
2009 \\
\mathrm{~N}=189 \\
\text { Female } 64 \%, \\
\text { male } 36 \% \text { mean } \\
\text { age (SD) } 50.0 \\
(11.9) \text { (self- } \\
\text { identified as } \\
\text { Hispanic/Latino) } \\
\text { Various } \\
\text { participants with } \\
\text { hyperlipidemia }\end{array}$ & $\begin{array}{l}\text { No theory } \\
\text { specified }\end{array}$ & $\begin{array}{l}\text { Participants assigned to } 1 \text { of } \\
3 \text { study arms: Community } \\
\text { health worker (CHW), case } \\
\text { management (CM) or } \\
\text { standard provider care (SPC) } \\
\text { CHW: } 3 \text { bilingual CHWs } \\
\text { delivered } 10 \text { weekly } \\
\text { education individual } \\
\text { sessions. Follow up } \\
\text { telephone calls. } \\
\text { CM: } 2 \text { registered nurses } \\
\text { worked with patients } \\
\text { individually for diabetes } \\
\text { education and monitoring. }\end{array}$ & $\begin{array}{l}\text { 3-group } \\
\text { randomized } \\
\text { control trial } \\
(\mathrm{RCT}) \text { with } 2 \\
\text { treatment and } \\
1 \text { control } \\
\text { groups } \\
\text { 6-month }\end{array}$ & $\begin{array}{l}\text { Exercise at least } 3 \\
\text { times per week } \\
\text { Self-reported PA }\end{array}$ & $\begin{array}{l}\text { CHW }(n=75) \text { Baseline } n=21(28 \%), 6 \text {-month } n \\
=47(63 \%), p<.05 \text { within groups at 6-month } \\
\text { follow-up } \\
\frac{\text { CM }(n=60) \text { Baseline } n=15(25 \%), 6 \text {-month } n=}{23(38 \%) p<.05 \text { between groups at 6-month }} \text { follow-up } \\
\frac{\text { SPC }(n=54) \text { Baseline } n=9(17 \%), 6 \text {-month } n=}{19(35 \%), p<.05 \text { within groups at 6-month }} \\
\text { follow-up } \\
\text { PA improved in CHW and SPC }\end{array}$ \\
\hline
\end{tabular}




\begin{tabular}{|c|c|c|c|c|c|}
\hline & & $\begin{array}{l}\text { Monthly visits and monthly } \\
\text { phone calls as needed } \\
\text { SPC: Usual care } \\
\text { Culturally appropriate } \\
\text { component: Incorporating } \\
\text { patient cultural and spiritual } \\
\text { beliefs for CHW group; using } \\
\text { culturally appropriate } \\
\text { educational materials based } \\
\text { on stages of change for CM } \\
\text { group }\end{array}$ & & & \\
\hline $\begin{array}{l}\text { Coleman et al., } \\
2012 \\
\mathrm{~N}=868 \\
\text { Females, mean } \\
\text { age } 52 \\
(65 \% \text { Mexican } \\
\text { and } 35 \% \\
\text { Central/South } \\
\text { American) } \\
\text { at risk for } \\
\text { developing } \\
\text { CVD, HTN or } \\
\text { hyperlipidemia }\end{array}$ & $\begin{array}{l}\text { Behavior } \\
\text { change theory }\end{array}$ & $\begin{array}{l}\text { Bilingual CHWs delivered } \\
\text { intervention of } 350 \text {-minute, } \\
\text { individually tailored } 1: 1 \\
\text { counseling sessions at } 1 \\
\text { month, } 2 \text { months, and } 6 \\
\text { months. } \\
\text { Culturally appropriate } \\
\text { component: Not specified }\end{array}$ & $\begin{array}{l}\text { 2-group RCT } \\
\text { 6-month }\end{array}$ & $\begin{array}{l}\text { Self-reported PA } \\
\text { Physical Activity } \\
\text { Assessment Survey }\end{array}$ & $\begin{array}{l}\text { Moderate PA CG Baseline } 328(75 \%), \text { Follow-up } \\
335(77 \%), \text { OR }(95 \% \text { CI) } 1.10(0.80-1.50), p= \\
.57 \\
\text { IG Baseline } 309 \text { (71\%), Follow-up 365 (84\%), } \\
\text { OR (95\% CI) 2.19 (1.57-3.07), } p<.001 \\
\text { Vigorous PA CG Baseline 69 (16\%) Follow-up } \\
75(17 \%), \text { OR (95\% CI) 1.11 }(0.77-1.59), p=.58 \\
\text { IG Baseline 57 (13\%), Follow-up 143 (33\%), OR } \\
\text { (95\% CI) 3.37 (2.38-4.77), } p<.001 \\
\text { IG group had significant results }\end{array}$ \\
\hline $\begin{array}{l}\text { Duggan et al., } \\
2014 \\
\mathrm{~N}=320 \\
\text { Female } 70.6 \%, \\
\text { male } 29.4 \%, \\
\text { mean age } 50.6 \\
\text { years (100\% } \\
\text { Hispanic/Latino) } \\
67 \% \text { TD2M } \\
\end{array}$ & $\begin{array}{l}\text { No theory } \\
\text { specified }\end{array}$ & $\begin{array}{l}\text { Educational curriculum } \\
\text { provided by CHWs at } \\
\text { participants' homes. } \\
5 \text { weekly guided sessions on } \\
\text { diabetes education } \\
\text { Culturally appropriate } \\
\text { component: Not specified }\end{array}$ & $\begin{array}{l}\text { 2-group RCT } \\
\text { 6-month }\end{array}$ & $\begin{array}{l}\text { Self-reported PA } \\
\text { IPAQ }\end{array}$ & $\begin{array}{l}\text { Leisure time PA mean (SE) } \\
\text { IG 3 months: Vigorous } 0.96(0.2), p=0.09 ; \\
\text { Moderate } 2.13(0.2), p=0.13 ; \text { Mild } 3.59(0.2), p \\
=0.72 \\
\text { CG } 3 \text { months: Vigorous } 0.72(0.1), \text { Moderate } \\
1.76(0.2), \text { Mild } 3.67(0.2) \\
\text { No significant results }\end{array}$ \\
\hline $\begin{array}{l}\text { Eakin et al. } 2007 \\
\mathrm{~N}=1356 \text { weeks }\end{array}$ & $\begin{array}{l}\text { Socio- } \\
\text { Ecological } \\
\text { Model and } \\
\text { behavioral- }\end{array}$ & $\begin{array}{l}\text { Health educator conducted } \\
\text { two f2f self-management } \\
\text { support and community } \\
\text { linkage sessions, } 60-90\end{array}$ & $\begin{array}{l}\text { 2- group RCT } \\
\text { 6-month }\end{array}$ & $\begin{array}{l}\text { Two PA outcome } \\
\text { variables: } \\
\text { Total min of } \\
\text { walking/week }\end{array}$ & $\begin{array}{l}\text { Change in minutes of walking/week: } \\
\text { IG: Baseline } 60(0-840) \text { total min walking/week } \\
\text { [median and range] }\end{array}$ \\
\hline
\end{tabular}




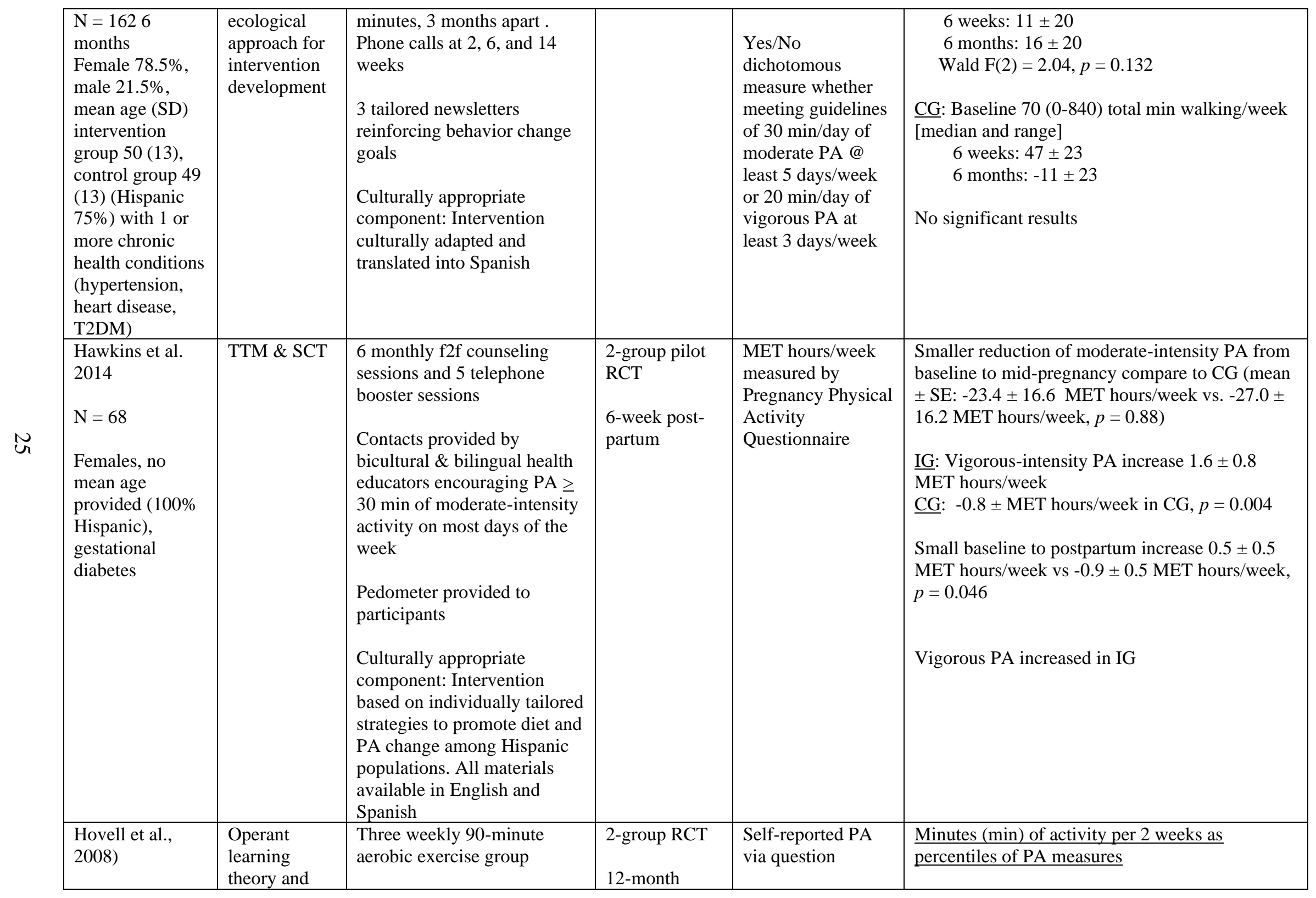




\begin{tabular}{|c|c|c|c|c|}
\hline $\begin{array}{l}\mathrm{N}=137 \\
\text { Females, mean } \\
\text { age (SD) } 31.36 \\
(6.21)(100 \% \\
\text { Hispanic, } \\
\text { country of origin } \\
\text { Mexico } 96.7 \% \text {, } \\
\text { other } 3.3 \%), \\
70 \% \text { of } \\
\text { participants } \\
\text { obese }\end{array}$ & $\begin{array}{l}\text { applied } \\
\text { behavior } \\
\text { analysis }\end{array}$ & $\begin{array}{l}\text { sessions for } 6 \text { months held in } \\
\text { a community setting. } \\
\text { Bilingual Latina aerobics } \\
\text { instructed led vigorous, low- } \\
\text { impact aerobic dance } \\
\text { sessions } \\
30 \text { minutes of exercise/diet } \\
\text { education with "hands-on" } \\
\text { learning activities } \\
\text { Culturally appropriate } \\
\text { component: Bilingual health } \\
\text { educators and guest speakers } \\
\text { provided culturally tailored, } \\
\text { low-literacy education on } \\
\text { home safety and selected } \\
\text { disease prevention topics } \\
\text { unrelated to exercise, diet, or } \\
\text { cardiovascular disease to CG. } \\
\text { Education component } \\
\text { following exercise segment } \\
\text { included exercise and diet } \\
\text { education with "hands-on" } \\
\text { learning activities culturally } \\
\text { appropriate for low-literacy } \\
\text { Latinas for IG. }\end{array}$ & $\begin{array}{l}\text { Direct PA } \\
\text { observation }\end{array}$ & 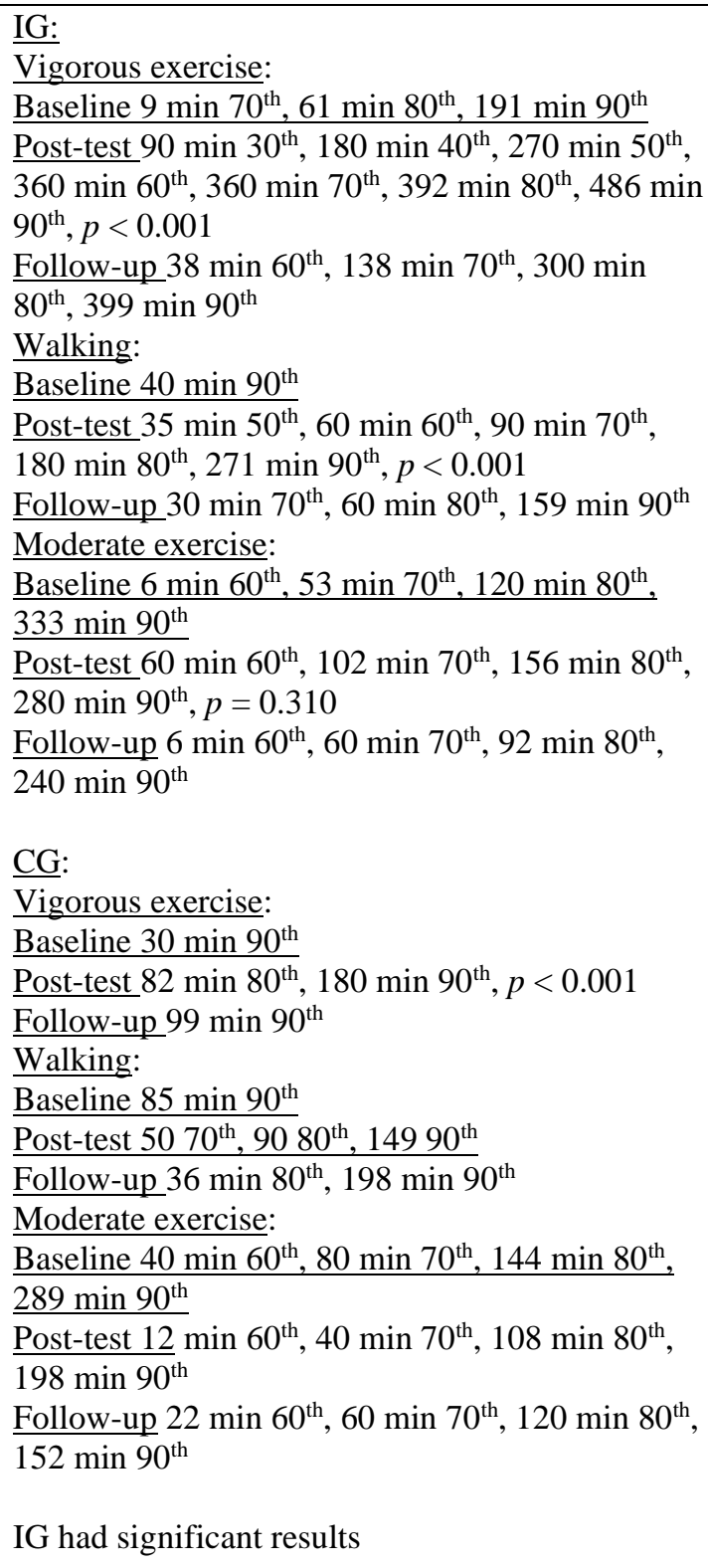 \\
\hline
\end{tabular}




\begin{tabular}{|c|c|c|c|c|c|}
\hline $\begin{array}{l}\text { Khare et al. } 2014 \\
\mathrm{~N}=155 \\
\text { Females, mean } \\
\text { age (SD) } 50.87 \\
\text { (6.88) (Mexican } \\
\text { or Central } \\
\text { American) }\end{array}$ & SCT \& TTM & $\begin{array}{l}\text { CVD risk factor screening, } \\
\text { related educational handout, } \\
\text { PRN physician care referral, } \\
\text { follow-up assessment @ } 1 \& \\
2 \text { years from baseline, } \\
\text { postcards and newsletter for } \\
\text { both groups } \\
\text { IG received 12-weekly } \\
\text { lifestyle change f2f } \\
\text { intervention sessions and } \\
\text { extra orientation session for } \\
\text { family members } \\
\text { Culturally appropriate } \\
\text { component: Not specified }\end{array}$ & $\begin{array}{l}\text { 2-group RCT } \\
\text { 12-month }\end{array}$ & $\begin{array}{l}\text { Community } \\
\text { Healthy Activities } \\
\text { Model Program for } \\
\text { Seniors } \\
\text { (CHAMPS) } \\
\text { summary scores } \\
\text { Hours/week in all } \\
\text { PA and hours/week }\end{array}$ & $\begin{array}{l}\text { Increases in all PA and moderate-intensity PA } \\
\text { within groups: } \\
\text { All PA: } \\
\text { IG: Baseline all PA } 8.45 \pm 5.36 \\
\text { Post-intervention } 13.53 \pm 6.59, \mathrm{~F}=20.94, p \\
<0.01 \\
1 \text { year } 8.48 \pm 5.73, \mathrm{~F}<0.01, p=0.99 \\
\text { CG: Baseline all PA } 8.81 \pm 5.18 \\
\text { Post-intervention } 11.11 \pm 7.74, \mathrm{~F}=3.89, p=0.05 \\
1 \text { year } 10.00 \pm 5.61, \mathrm{~F}=0.72, p=0.40 \\
\text { Moderate Intensity PA: } \\
\text { IG: Baseline all PA } 2.57 \pm 2.89 \\
\text { Post-intervention } 5.06 \pm 3.73, \mathrm{~F}=15.95, p<0.01 \\
1 \text { year } 3.26 \pm 3.17, \mathrm{~F}=0.79, p=0.38 \\
\text { CG: Baseline all PA } 2.99 \pm 3.33 \\
\text { Post-intervention } 4.41 \pm 4.92, \mathrm{~F}=3.59, p=0.06 \\
1 \text { year } 3.81 \pm 3.87, \mathrm{~F}=0.57, p=0.45 \\
\text { No significant results }\end{array}$ \\
\hline $\begin{array}{l}\text { King et al., } 2013 \\
\mathrm{~N}=39 \\
\text { Female } 65 \%, \\
\text { male } 35 \% \text { mean } \\
\text { age (SD) } 72.1 \\
\text { (8.2) (92.5\% } \\
\text { Hispanic, } \\
\text { country of origin } \\
40 \% \text { Mexico, } \\
40 \% \text { U.S., } 20 \% \\
\text { other) with } \\
\text { previous health } \\
\text { conditions }\end{array}$ & TTM \& SCT & $\begin{array}{l}\text { 4-month embodied } \\
\text { conversational agent (ECA) } \\
\text { PA intervention delivered } \\
\text { through a computer in a local } \\
\text { community senior center. } \\
\text { Participants encouraged to } \\
\text { complete virtual advisor } \\
\text { sessions regularly } \\
\text { Pedometer provided to } \\
\text { participants } \\
\text { Culturally appropriate } \\
\text { component: } 12 \text { months of }\end{array}$ & $\begin{array}{l}\text { 2-group RCT } \\
\text { 4-month }\end{array}$ & $\begin{array}{l}\text { CHAMPS } \\
\text { Questionnaire } \\
\text { Walking changes at } \\
\text { baseline, } 2 \text { and } 4 \\
\text { months }\end{array}$ & $\begin{array}{l}\text { 4-month changes in min/walking/week } \\
\text { CG: } M \text { change } 26.8(67.0) \\
\text { Virtual advisor IG: } M \text { change } 253.5(248.7) \\
\text { Between-group difference: } 226.8,95 \% \text { CI } 107.0 \\
\text {-346.4 } F(1,38)=13.6, p=.0008 \\
\text { IG had significantly more steps }\end{array}$ \\
\hline
\end{tabular}




\begin{tabular}{|c|c|c|c|c|c|}
\hline & & $\begin{array}{l}\text { participatory formative } \\
\text { research and evaluation to } \\
\text { enhance ECA cultural and } \\
\text { linguistic congruity for } \\
\text { Latino and, to some extent, } \\
\text { Filipino older adults. }\end{array}$ & & & \\
\hline $\begin{array}{l}\text { Koniak-Griffin } \\
\text { et al., } 2014 \\
\mathrm{~N}=223 \\
\text { Females, mean } \\
\text { age (SD) } 44.6 \\
\text { (7.9) (84\% } \\
\text { Mexican, 14\% } \\
\text { Dominican, } \\
\text { Central or South } \\
\text { American, 2\% } \\
\text { born in US but } \\
\text { raised in } \\
\text { Mexico), with } \\
\text { T2DM and } \\
\text { hypertension }\end{array}$ & $\begin{array}{l}\text { No theory } \\
\text { specified }\end{array}$ & $\begin{array}{l}\text { Mujeres Sanas y Precavidas } \\
\text { 6-month lifestyle intervention } \\
\text { comprised of group education } \\
\text { plus individual teaching and } \\
\text { coaching } \\
\text { Eight } 2 \text {-hour weekly classes } \\
\text { for } 1^{\text {st }} 2 \text { months. } \\
\text { Individual teaching and } \\
\text { coaching had } 8 \text { contacts } \\
\text { delivered over } 4 \text { months ( } 4 \\
\text { home visits plus } 4 \text { telephone } \\
\text { calls).Personal goals } \\
\text { established by participants } \\
\text { for lifestyle changes. } \\
\text { Messages included increasing } \\
\text { PA to } 10,000 \text { steps per day; } \\
\text { PA diaries provided } \\
\text { Accelerometer provided to } \\
\text { participants } \\
\text { Culturally appropriate } \\
\text { component: Classes based on } \\
\text { culturally relevant, } \\
\text { promotora-led educational } \\
\text { program developed for } \\
\text { Latino communities by } \\
\text { National Heart, Lung, and } \\
\text { Blood Institute }\end{array}$ & $\begin{array}{l}\text { 2-group RCT } \\
\text { 9-month }\end{array}$ & $\begin{array}{l}\text { Daily steps via } \\
\text { accelerometer }\end{array}$ & $\begin{array}{l}\text { IG: Baseline } 8579 \pm 3268 \\
6 \text { month } 8769 \pm 2747 \\
9 \text { month } 8577 \pm 2872 \\
\text { Contrast } \mathrm{t}=2.07, \mathrm{df}=201, p=0.04 \\
\text { CG: Baseline } 8571+3130 \\
6 \text { month } 8480+3506 \\
9 \text { month } 7241+2764 \\
\text { IG had more steps }\end{array}$ \\
\hline $\begin{array}{l}\text { Marcus et al., } \\
2016\end{array}$ & SCT \& TTM & $\begin{array}{l}\text { Participants provided with } \\
\text { study website access }\end{array}$ & $\begin{array}{l}\text { 2-group RCT } \\
\text { 6-month }\end{array}$ & $\begin{array}{l}\text { Accelerometer PA } \\
\text { total min/week }\end{array}$ & $\begin{array}{l}\text { IG: Baseline } 8.0 \text { (SD 15.0) } \mathrm{min} / \text { week MVPA } \\
6 \text { Month 112.8 (SD 97.1) min/week MVPA }\end{array}$ \\
\hline
\end{tabular}




\begin{tabular}{|c|c|c|c|c|c|}
\hline $\begin{array}{l}\mathrm{N}=218 \\
\text { Females, mean } \\
\text { age (SD) } 39.2 \\
(10.47)(87.3 \% \\
\text { Hispanic, } 84.4 \% \\
\text { Mexican } \\
\text { American, } 3.4 \% \\
\text { Colombian, } \\
1.0 \% \\
\text { Guatemalan, } \\
1.0 \% \text { Puerto } \\
\text { Rican, } 0.5 \% \\
\text { Dominican } \\
\text { Republic, } 12.7 \% \\
\text { other }\end{array}$ & & $\begin{array}{l}\text { including self-monitoring of } \\
\text { minutes of activity and steps. } \\
\text { Components included goal } \\
\text { setting, message board for } \\
\text { support, "ask the expert," } \\
\text { maps for walking routes, and } \\
\text { exercise videos } \\
\text { Monthly questionnaires } \\
\text { generated tailored PA reports } \\
\text { including information on } \\
\text { current stage of motivational } \\
\text { readiness for PA, comparison } \\
\text { of participants to other } \\
\text { individuals that are } \\
\text { physically active, comparison } \\
\text { of participant responses to } \\
\text { previous responses, and } \\
\text { useful PA facts } \\
\text { Weekly email prompts to } \\
\text { access intervention website in } \\
\text { month 1, biweekly in months } \\
2 \text { and 3, and monthly during } \\
\text { months 4-6 } \\
\text { Culturally appropriate } \\
\text { component: Non-specific } \\
\text { culturally and linguistically } \\
\text { adapted, individually } \\
\text { tailored, Internet-based PA } \\
\text { intervention }\end{array}$ & & $\begin{array}{l}\text { 7-Day PAR } \\
\text { Questionnaire }\end{array}$ & $\begin{array}{l}\text { CG: Baseline 10.44 (SD 23.98) min/week MVPA } \\
6 \text { Month 63.5 (SD 88.7) min/week MVPA } \\
\text { Adjusted model } \\
\text { IG had 50.0 more min/week of MVPA at } 6 \\
\text { months compared to CG (adjusting for baseline), } \\
\text { SE }=9.5, p=.01 \\
\text { IG had more steps }\end{array}$ \\
\hline $\begin{array}{l}\text { Marcus et al., } \\
2013 \\
\mathrm{~N}=266\end{array}$ & TTM \& SCT & $\begin{array}{l}\text { Print interventions to increase } \\
\text { PA, } 4 \text { mailings in month } 1,2 \\
\text { mailings in months } 2-3 \text {, and } 1 \\
\text { mailing in months } 4-6\end{array}$ & $\begin{array}{l}\text { 2-group RCT } \\
\text { 6-month }\end{array}$ & $\begin{array}{l}3 \text { psychosocial } \\
\text { measures at } \\
\text { baseline, } 3 \text { months } \\
\text { via mail, and } 6 \\
\text { months f2f to }\end{array}$ & $\begin{array}{l}\text { Mean (SD) min/week of MVPA } \\
\text { IG: } 6 \text { months } 73.36(89.73) M 41.36 \text { (SE 7.93), } p \\
<0.01 \\
\text { CG: } 6 \text { months } 32.98(82.82)\end{array}$ \\
\hline
\end{tabular}




\begin{tabular}{|c|c|c|c|c|c|}
\hline $\begin{array}{l}\text { Females, mean } \\
\text { age (SD) } 41.61 \\
\text { (10.07) }(34.1 \% \\
\text { Dominican, } \\
\text { Colombian } \\
30.3 \%, 10.6 \% \\
\text { Puerto Rican, } \\
6.8 \% \\
\text { Guatemalan, } \\
5.3 \% \text { Mexican, } \\
\text { American or } \\
\text { Chicana, other } \\
12.9 \% \text {, with } \\
\text { increased BMI }\end{array}$ & & $\begin{array}{l}\text { Pedometer provided to } \\
\text { participants } \\
\text { Culturally appropriate } \\
\text { component: Formative } \\
\text { research conducted to } \\
\text { culturally and linguistically } \\
\text { adapt intervention for } \\
\text { Latinas, and adapted } \\
\text { intervention tested in a pilot } \\
\text { study }\end{array}$ & & $\begin{array}{l}\text { develop } \\
\text { intervention } \\
\text { 7-Day PAR } \\
\text { Questionnaire }\end{array}$ & IG had significant results \\
\hline $\begin{array}{l}\text { Marshall et al., } \\
2013 \\
\mathrm{~N}=180 \\
\text { Females, mean } \\
\text { age (SD) } \\
\text { intervention } 1 \\
36.94 \text { (8.86), } \\
\text { intervention } 2 \\
35.27 \text { (8.76), } \\
\text { intervention } 3 \\
35.42 \text { (8.41) } \\
\text { (100\% Latina, } \\
\text { country of origin } \\
\text { intervention } 1 \\
\text { Mexico 98.2\%, } \\
\text { US 1.8\%, } \\
\text { intervention } 2 \\
\text { Mexico 94\%, } \\
\text { U.S. 2\%, other } \\
4 \% \text {, intervention } \\
3 \text { Mexico } 94.6 \%, \\
\text { U.S. 3.4\%, with } \\
\text { increased BMI }\end{array}$ & $\begin{array}{l}\text { Behavioral } \\
\text { and } \\
\text { socioecologic } \\
\text { al models for } \\
\text { Latino health } \\
\text { promotion } \\
\text { and } \\
\text { Communicati } \\
\text { on Persuasion } \\
\text { Model }\end{array}$ & $\begin{array}{l}\text { 12-week Spanish language } \\
\text { PA program (Pasos } \\
\text { Adelante). Weekly 1-hour } \\
\text { group meetings led by a } \\
\text { promotora } \\
\text { Pedometers provided to } \\
\text { participants } \\
\text { Intervention } 1 \text { self-selected } \\
\text { step goal, intervention } 2 \\
10,000 \text { steps/day, } \\
\text { intervention } 33,000 \text { steps-in- } \\
30 \text { minutes } \\
\text { Culturally appropriate } \\
\text { component: Culturally } \\
\text { targeted and tailored flyers, } \\
\text { letters, friend referral, } \\
\text { presentations at community } \\
\text { gatherings and parent-teacher } \\
\text { meetings, and incentives to } \\
\text { follow recommendations for } \\
\text { recruiting minorities and } \\
\text { underserved populations }\end{array}$ & $\begin{array}{l}\text { 3-group RCT } \\
\text { with } 2 \\
\text { treatment and } \\
1 \text { control } \\
\text { group } \\
\text { 3-month }\end{array}$ & $\begin{array}{l}\text { Accelerometer- } \\
\text { based PA measured } \\
\text { at baseline and } \\
\text { after } 12 \text { weeks }\end{array}$ & 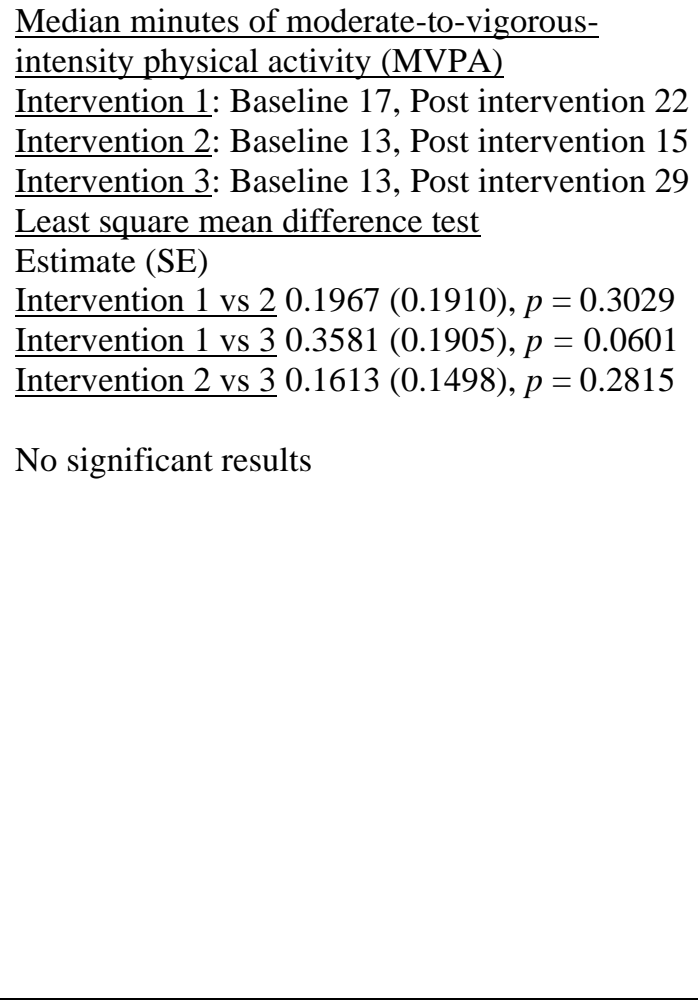 \\
\hline
\end{tabular}




\begin{tabular}{|c|c|c|c|c|c|}
\hline $\begin{array}{l}\text { McEwen et al., } \\
2017 \\
\mathrm{~N}_{\mathrm{T} 1}=157 \\
\mathrm{~N}_{\mathrm{T} 2}=105 \\
\mathrm{~N}_{\mathrm{T} 3}=87 \\
\text { Participant } \\
\text { Female 65\%, } \\
\text { male 35\%, mean } \\
\text { age (SD) 53.53 } \\
(9.0) \text { (Mexican- } \\
\text { American 100\%) } \\
\text { with T2DM, } \\
\text { increased BMI } \\
\\
\text { Family } \\
\text { Female 72.6\%, } \\
\text { male 27.4\%, } \\
\text { mean age (SD) } \\
47.27 \text { (16.1) } \\
\text { (Mexican- } \\
\text { American 100\%) } \\
\text { with increased } \\
\text { BMI }\end{array}$ & $\begin{array}{l}\text { No theory } \\
\text { specified }\end{array}$ & $\begin{array}{l}\text { 12-week intervention } \\
\text { program with six weekly 2- } \\
\text { hour education (led by a } \\
\text { certified diabetes educator } \\
\text { registered nurse) and social } \\
\text { support (led by promotoras) } \\
\text { sessions focused on } \\
\text { managing diabetes. } \\
\text { Three weekly 2-hour home } \\
\text { visits and three 20-minute } \\
\text { phone calls completed by } \\
\text { promotoras. } \\
\text { Goals established in group } \\
\text { sessions and } \\
\text { evaluated/refined as needed } \\
\text { in home visits. Phone calls } \\
\text { completed to check } \\
\text { participants' progress or } \\
\text { barriers in meeting goals. } \\
\text { Wait-list: 2-hour education } \\
\text { sessions weekly for } 3 \text { weeks } \\
\text { Culturally appropriate } \\
\text { component: Mexican } \\
\text { American participants with } \\
\text { T2DM and family members } \\
\text { participated in focus groups } \\
\text { to inform intervention using } \\
\text { community-based } \\
\text { participatory research } \\
\text { principles }\end{array}$ & $\begin{array}{l}\text { 2-group } \\
\text { experimental } \\
\text { repeated } \\
\text { measures with } \\
\text { dyad cohorts } \\
\text { and wait list } \\
\text { control } \\
\text { 6-month }\end{array}$ & $\begin{array}{l}\text { IPAQ } \\
\text { MET minutes per } \\
\text { week }\end{array}$ & 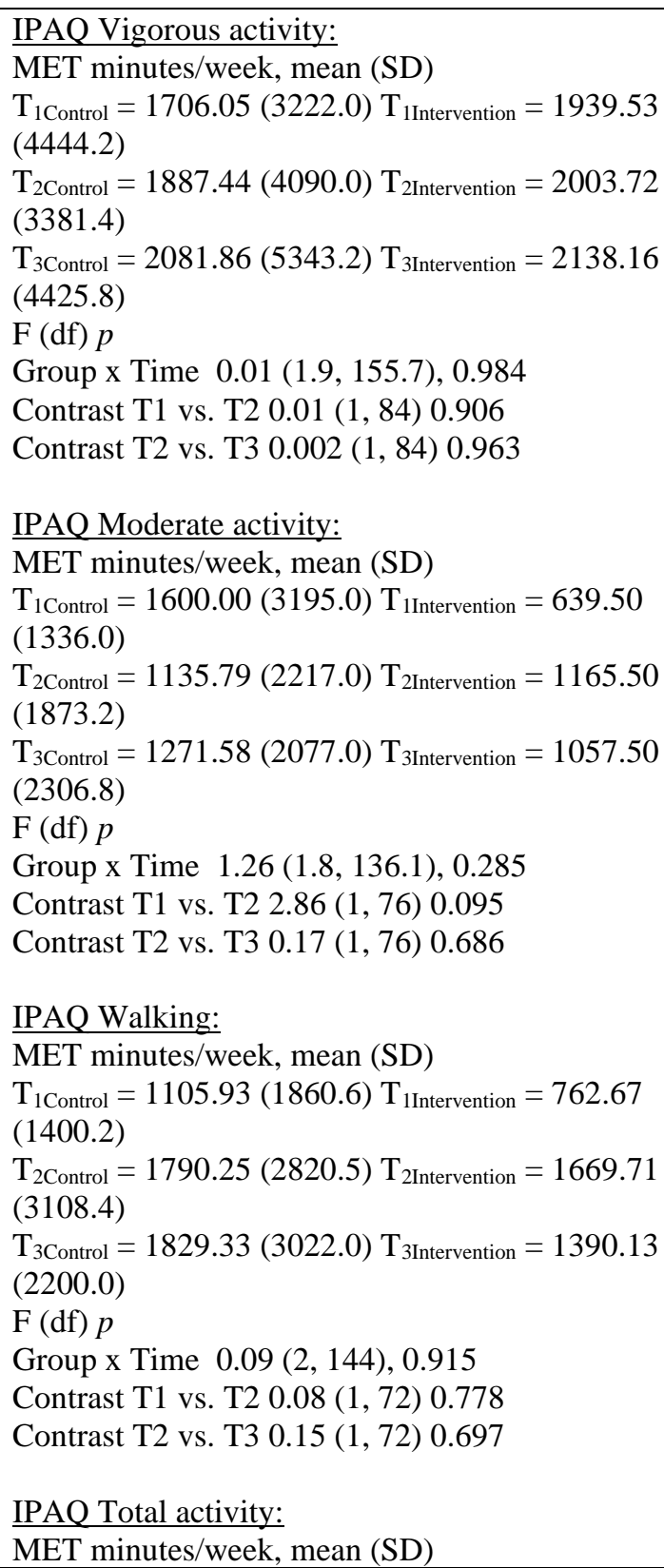 \\
\hline
\end{tabular}




\begin{tabular}{|c|c|c|c|c|c|}
\hline & & & & & $\begin{array}{l}\mathrm{T}_{1 \text { Control }}=3877.17(5284.2) \mathrm{T}_{1 \text { Intervention }}=4278.16 \\
(7291.3) \\
\mathrm{T}_{2 \text { Control }}=4406.01(7090.4) \mathrm{T}_{\text {2Intervention }}=4874.10 \\
(6803.7) \\
\mathrm{T}_{3 \text { Control }}=5027.09(8791.5) \mathrm{T}_{3 \text { Intervention }}=4322.76 \\
(7294.5) \\
\mathrm{F}(\mathrm{df}) p \\
\text { Group x Time } 0.25(2,190), 0.783 \\
\text { Contrast T1 vs. T2 } 0.001(1,95) 0.970 \\
\text { Contrast T2 vs. T3 } 0.38(1,95) 0.538 \\
\text { No significant results }\end{array}$ \\
\hline $\begin{array}{l}\text { Mitchell et al., } \\
2015 \\
\mathrm{~N}=254 \\
\text { Female } 72 \%, \\
\text { male } 28 \%, \text { mean } \\
\text { age (SD) } 33.2 \\
\text { (7.8) female, } \\
30.2(7.0) \text { male } \\
\text { (Latino } 100 \% \text { ), } \\
\text { with increased } \\
\text { BMI }\end{array}$ & $\begin{array}{l}\text { No theory } \\
\text { specified }\end{array}$ & $\begin{array}{l}\text { Ten weekly } 90 \text {-minute } \\
\text { sessions with PA for } 15-20 \\
\text { minutes, with participants } \\
\text { sharing a healthy snack and } \\
\text { promising to improve } \\
\text { lifestyle } \\
\text { Culturally appropriate } \\
\text { component: Use of a } \\
\text { Mexican specialist to create } \\
\text { intervention program and } \\
\text { development of culturally } \\
\text { and linguistically appropriate } \\
\text { low-literacy materials }\end{array}$ & $\begin{array}{l}\text { RCT } \\
\text { 12-14 week }\end{array}$ & $\begin{array}{l}\text { PA changes in } \\
\text { days/week } \\
\text { performing at least } \\
30 \text { min of PA, } \\
\text { days/week of at } \\
\text { least } 10 \text { minutes of } \\
\text { PA producing } \\
\text { moderate heart and } \\
\text { respiratory rate } \\
\text { increases, and } \\
\text { days/week of at } \\
\text { least } 10 \text { minutes of } \\
\text { PA producing large } \\
\text { increases in heart } \\
\text { and respiratory rate } \\
\\
\text { PA Confidence } \\
\text { Scales and } \\
\text { Behavioral Risk } \\
\text { Factor Surveillance } \\
\text { System } 2009 \\
\text { questionnaire }\end{array}$ & 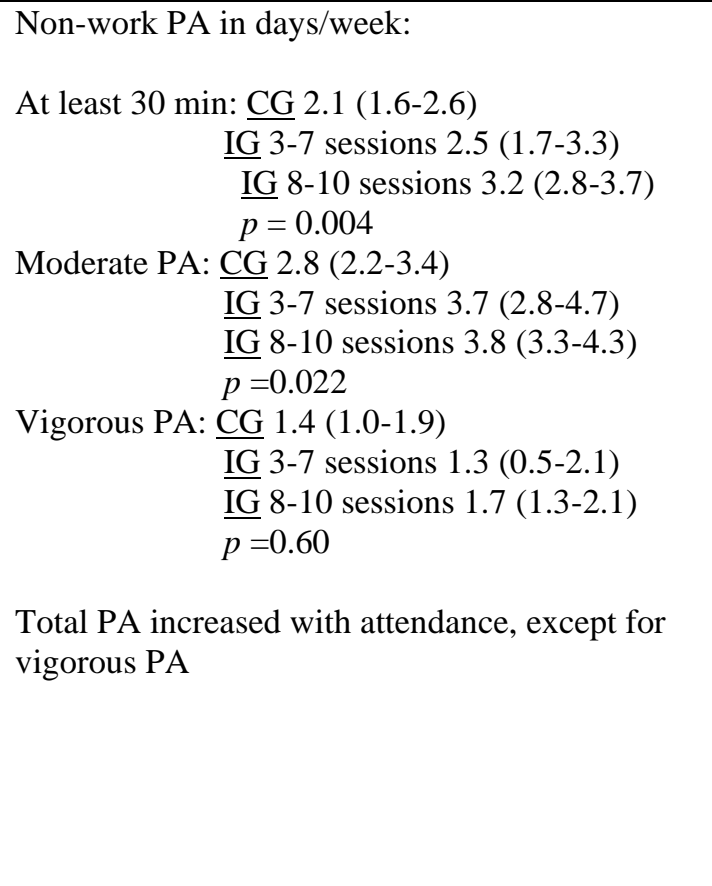 \\
\hline $\begin{array}{l}\text { Pekmezi et al., } \\
2009\end{array}$ & TTM \& SCT & $\begin{array}{l}\text { IG: Six monthly PA manual } \\
\text { mailings matched to } \\
\text { participants' current level of }\end{array}$ & $\begin{array}{l}\text { 2-group RCT } \\
\text { 6-month }\end{array}$ & 7-Day PAR & $\frac{\text { Mean min/week of at least moderate-intensity PA }}{M(\mathrm{SD})}$ \\
\hline
\end{tabular}




\begin{tabular}{|c|c|c|c|c|c|}
\hline $\begin{array}{l}\mathrm{N}=93 \\
\text { Females, mean } \\
\text { age (SD) } 41.37 \\
(11.18) \\
\text { (Dominican } \\
35 \% \text {, Colombian } \\
31 \% \text {, Puerto } \\
\text { Rican 11\%, } \\
\text { Guatemalan } \\
\text { 10\%, other 13\%) } \\
\text { with increased } \\
\text { BMI }\end{array}$ & & $\begin{array}{l}\text { motivational readiness and } \\
\text { individually tailored } \\
\text { computer expert-system } \\
\text { feedback reports. } \\
\text { CG: Six monthly mailings of } \\
\text { health information on topics } \\
\text { other than PA } \\
\text { Culturally appropriate } \\
\text { component: Cultural and } \\
\text { linguistical adaptation of an } \\
\text { existing empirically } \\
\text { supported, computer-tailored } \\
\text { PA intervention. Formative } \\
\text { research on individually } \\
\text { tailored PA print intervention } \\
\text { conducted by } \\
\text { bilingual/bicultural staff. }\end{array}$ & & & $\begin{array}{l}\text { IG Baseline } 16.56 \text { (25.76), } 6 \text { months } 147.27 \\
(241.55) \\
\mathrm{F}(1,91)=1.37, p=0.25 \\
\text { CG Baseline } 11.88(21.99), 6 \text { months } 96.79 \\
\text { (118.49) } \\
\text { No significant results }\end{array}$ \\
\hline $\begin{array}{l}\text { Poston et al., } \\
2001 \\
\mathrm{~N}=379 \\
\text { Females, mean } \\
\text { age (SD) IG } 39.2 \\
\text { (10.6), CG 40.0 } \\
\text { (6.4) (Mexican- } \\
\text { American } \\
100 \% \text { ), with } \\
\text { increased BMI }\end{array}$ & $\underline{\mathrm{SCT}}$ & $\begin{array}{l}\text { Weekly } 90 \text {-minute meetings } \\
\text { for } 6 \text { months led by bilingual } \\
\text { Mexican-American health } \\
\text { professionals } \\
\text { Content used individually } \\
\text { oriented behavioral } \\
\text { techniques with culturally } \\
\text { compatible strategies } \\
\text { Culturally appropriate } \\
\text { content: Use of bilingual } \\
\text { materials and instructors/peer } \\
\text { leaders, modification of } \\
\text { native diets, culturally } \\
\text { tailored rationales for diet } \\
\text { and activity modification, } \\
\text { and use of established social } \\
\text { support networks }\end{array}$ & $\begin{array}{l}\text { 2-group RCT } \\
\text { 12-month }\end{array}$ & 7-Day PAR & 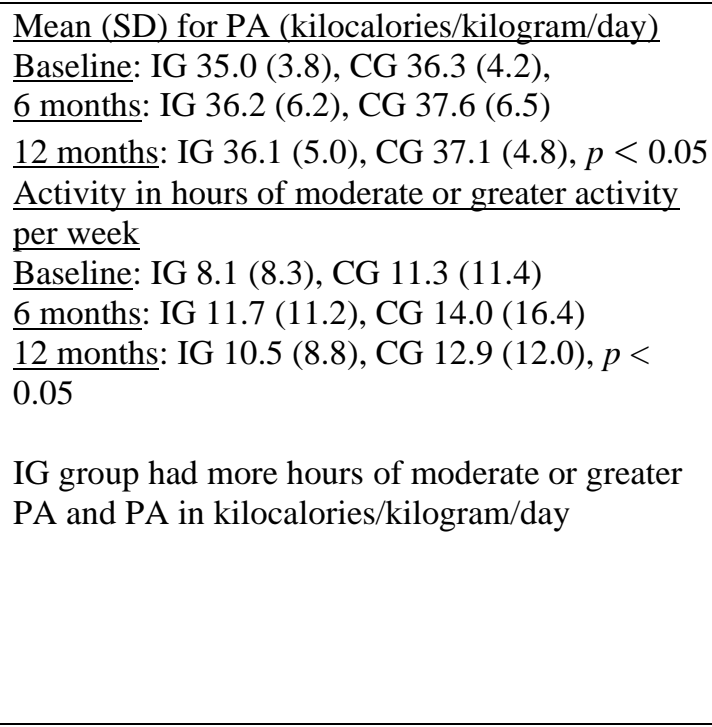 \\
\hline $\begin{array}{l}\text { Rosal et al. } 2011 \\
\mathrm{~N}=252\end{array}$ & $\underline{\mathrm{SCT}}$ & $\begin{array}{l}12 \text { weekly sessions and } \\
\text { follow up phase of } 8 \text { monthly } \\
\text { sessions. Week } 160 \text {-minute }\end{array}$ & 2-group RCT & $\begin{array}{l}\text { PA Survey } \\
\text { question }\end{array}$ & Self-report walking for exercise \\
\hline
\end{tabular}




\begin{tabular}{|c|c|c|c|c|c|}
\hline $\begin{array}{l}\text { Female } 77 \%, \\
\text { male } 23 \%, \text { age } \\
18-4416.3 \%, \\
45-5429.8 \%, \\
55-6432.9 \%, \geq \\
6521.0 \% \\
\text { (Puerto Rican } \\
87.7 \% \text { ), with } \\
\text { T2DM }\end{array}$ & & $\begin{array}{l}\text { session at participant's home. } \\
\text { Subsequent weekly } 150- \\
\text { minute sessions in groups at } \\
\text { community settings, with } \\
\text { first } 60 \text { minutes of } \\
\text { personalized counseling and } \\
\text { cooking, and last } 90 \text { minutes } \\
\text { of group protocol and meal. } \\
\text { Sessions delivered by } \\
\text { nutritionist, health educator, } \\
\text { or trained lay person } \\
\text { Pedometer provided to } \\
\text { participants } \\
\text { Culturally appropriate } \\
\text { component: Cultural } \\
\text { tailoring included use of an } \\
\text { educational soap opera to } \\
\text { introduce self-management } \\
\text { information and model } \\
\text { attitudinal change and desired } \\
\text { behaviors in the context of } \\
\text { culturally relevant situations, } \\
\text { use of bingo games to } \\
\text { reinforce information taught, } \\
\text { emphasis on making } \\
\text { traditional foods healthier via } \\
\text { healthy preparation methods, } \\
\text { and addressing family } \\
\text { preferences. }\end{array}$ & 12-month & $\begin{array}{l}\text { MET or total time } \\
\text { of walking, sitting, } \\
\text { or PA }\end{array}$ & $\begin{array}{l}\text { IG: Baseline } 61.3 \% \\
\text { 4-month } 88.4 \% p=0.057 \\
\text { 12-month } 70.9 \% p=0.435 \\
\text { CG: Baseline } 52.3 \% \\
\text { 4-month } 70.9 \% p=0.057 \\
\text { 12-month } 66.4 \% p=0.435 \\
\text { No significant results reported for PA. }\end{array}$ \\
\hline $\begin{array}{l}\text { Rosal et al., } 2005 \\
\mathrm{~N}=25 \\
\text { Female } 80 \% \text {, } \\
\text { male } 20 \% \text {, mean } \\
\text { age (SD) } 62.6 \\
\text { (8.6) (Puerto } \\
\text { Rican } 100 \% \text {, } \\
\text { with DMT2 }\end{array}$ & $\underline{\mathrm{SCT}}$ & $\begin{array}{l}\text { Individual initial 60-minute } \\
\text { session and two } 15 \text {-minute } \\
\text { individual sessions preceding } \\
\text { group sessions } \\
\\
\text { Ten weekly 150-180-minute } \\
\text { group sessions targeting } \\
\text { diabetes knowledge, } \\
\text { attitudes, and self- }\end{array}$ & $\begin{array}{l}\text { 2-group pilot } \\
\text { RCT } \\
\text { 6-month }\end{array}$ & $\begin{array}{l}\text { CHAMPS } \\
\text { Questionnaire } \\
\text { (modified version) }\end{array}$ & $\begin{array}{l}\text { Kilocalories/week mean (SD) } \\
\text { IG: baseline } 660(705) \\
\text { 3-month } 439(935) \\
\quad \text { 6-month } 272(929) \\
\quad p=0.11 \\
\text { CG: baseline } 976(1154) \\
\text { 3-month -180 (1262) }\end{array}$ \\
\hline
\end{tabular}




\begin{tabular}{|c|c|c|c|c|c|}
\hline & & $\begin{array}{l}\text { management skills. Delivered } \\
\text { by diabetes nurse, } \\
\text { nutritionist, and assistant } \\
\text { Booklet describing } \\
\text { importance of lifestyle } \\
\text { factors and recommendations } \\
\text { for diet, PA, and self- } \\
\text { monitoring blood glucose } \\
\text { Drama (soap opera) } \\
\text { developed to convey } \\
\text { messaged discussed at each } \\
\text { session } \\
\text { Culturally appropriate } \\
\text { component: Intervention } \\
\text { tailored to low-literacy needs } \\
\text { and imparted through } \\
\text { culturally familial } \\
\text { experiences in English and } \\
\text { Spanish. }\end{array}$ & & & $\begin{array}{l}\quad \begin{array}{l}\text { 6-month -512 (967) } \\
\quad p=0.11\end{array} \\
\text { No significant results }\end{array}$ \\
\hline $\begin{array}{l}\text { Rothschild et al., } \\
2014 \\
\mathrm{~N}=144 \\
\text { Female } 67.4 \%, \\
\text { male } 32.6 \% \text {, } \\
\text { mean age (SD) } \\
53.7(12.2) \\
\text { (Mexican- } \\
\text { American } \\
100 \% \text { ), with } \\
\text { T2DM }\end{array}$ & $\begin{array}{l}\text { Self- } \\
\text { Management } \\
\text { Theory }\end{array}$ & $\begin{array}{l}\text { Baseline evaluations in } 2 \\
\text { separate encounters } \\
\text { approximately } 30 \text { days apart } \\
36 \text { home visits over } 2 \text { years, } \\
\text { average visit duration } 99 \\
\text { minutes. CHWs delivered } \\
\text { behavioral self-management } \\
\text { training. Sessions focused on } \\
\text { knowledge and skills in } \\
\text { diabetes self-management } \\
\text { with goal-setting. } \\
\text { Culturally appropriate } \\
\text { content: CHWs taught in the } \\
\text { participants' preferred }\end{array}$ & $\begin{array}{l}\text { 2-group RCT } \\
\text { 24-month }\end{array}$ & $\begin{array}{l}\text { PA Subscales of } \\
\text { Diabetes Self-Care } \\
\text { Activities and } \\
\text { Diabetes } \\
\text { Empowerment } \\
\text { Scale } \\
\text { Mean days/week of } \\
\text { PA }\end{array}$ & $\begin{array}{l}\text { IG: Baseline } 1.63 \text { days/week } \\
\text { 2-year } 2.64 \text { days/week }\end{array}$ \\
\hline
\end{tabular}




\begin{tabular}{|c|c|c|c|c|}
\hline & & $\begin{array}{l}\text { language and used culturally } \\
\text { appropriate examples or } \\
\text { metaphors }\end{array}$ & & \\
\hline $\begin{array}{l}\text { Vincent } 2009 \\
\mathrm{~N}=17 \\
\text { Female } 71 \%, \\
\text { male } 29 \%, \text { mean } \\
\text { age } 56 \\
\text { (Mexican- } \\
\text { American } \\
\text { 100\%), with } \\
\text { T2DM }\end{array}$ & $\begin{array}{l}\text { Chronic } \\
\text { Disease Self- } \\
\text { Management } \\
\text { Model based } \\
\text { on SCT }\end{array}$ & $\begin{array}{l}\text { Eight weekly 120-minute } \\
\text { sessions } \\
\text { Sessions comprised of } \\
\text { didactic content related to } \\
\text { diabetes and taught by } \\
\text { bilingual certified diabetes } \\
\text { educator. Also, promotoras } \\
\text { led cooking demonstrations, } \\
\text { and group sessions as well as } \\
\text { calling participants weekly } \\
\text { Pedometers provided to } \\
\text { participants } \\
\text { Culturally appropriate } \\
\text { component: Content } \\
\text { included low fat } \\
\text { modifications of traditional } \\
\text { foods, discussing use of } \\
\text { traditional home remedies, } \\
\text { and emphasizing culturally } \\
\text { appropriate exercise } \\
\text { strategies such as walking } \\
\text { and dancing for Mexican } \\
\text { Americans. Participants were } \\
\text { also encouraged to bring a } \\
\text { family member as a support } \\
\text { person. Participants received } \\
\text { low-literacy materials in } \\
\text { Spanish to share with family } \\
\text { members }\end{array}$ & $\begin{array}{l}\text { 2-group RCT } \\
\text { PA measure: } \\
\text { step count with } \\
\text { pedometer, } \\
\text { daily record on } \\
\text { log } \\
\text { 3-month f/u }\end{array}$ & 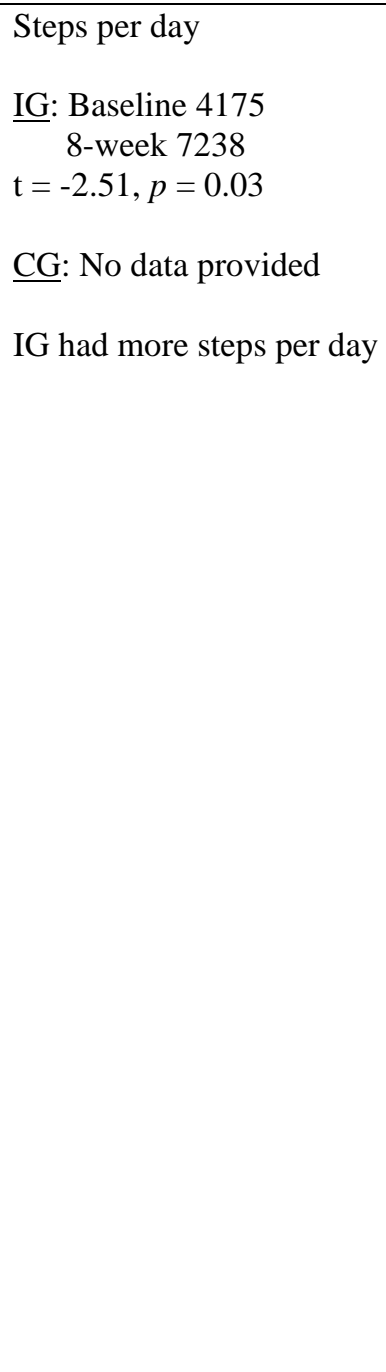 \\
\hline
\end{tabular}


BMI: Body mass index, IPAQ: International Physical Activity Questionnaire, f2f: face-to-face, MET: metabolic equivalent task, MVPA: moderate-to-vigorous physical activity, 7-Day PAR: 7-Day Physical Activity Recall, PA: physical activity, SCT: Social Cognitive Theory, SD: standard deviation, SE: standard error, T2DM: type 2 diabetes mellitus, TTM: Transtheoretical Model 
Table 2.2

Outcome measures used in studies

\begin{tabular}{|c|c|}
\hline PA measure & Study \\
\hline Days per week of PA & (Rothschild et al., 2014) \\
\hline Exercise at least 3 times per week & (Babamoto et al., 2009) \\
\hline $\begin{array}{l}\text { Metabolic equivalent minutes of leisure-time } \\
\text { and weekly PA }\end{array}$ & (Hawkins et al., 2015; McEwen et al., 2017) \\
\hline $\begin{array}{l}\text { Number of days participants completed } 30 \\
\text { minutes of PA }\end{array}$ & (Mitchell et al., 2015) \\
\hline Percentage of participants reporting MVPA & (Coleman et al., 2012) \\
\hline $\begin{array}{l}\text { Difference in leisure-time mild, moderate, and } \\
\text { vigorous PA }\end{array}$ & (Duggan et al., 2014) \\
\hline $\begin{array}{l}\text { Percentiles of participants engaging in } \\
\text { walking, moderate, and vigorous exercise }\end{array}$ & $\begin{array}{l}\text { (Coleman et al., 2012; Duggan et al., 2014; } \\
\text { Hovell et al., 2008) }\end{array}$ \\
\hline Minutes of walking per week & (Eakin et al., 2007; Rosal et al., 2011) \\
\hline Minutes of walking per day & (Koniak-Griffin et al., 2015) \\
\hline Minutes of MVPA & (Marshall et al., 2013) \\
\hline $\begin{array}{l}\text { MET hours per day and total PA duration in } \\
\text { hours per day }\end{array}$ & (Rosal et al., 2011) \\
\hline Change in weekly MVPA in minutes & $\begin{array}{l}\text { (Arredondo et al., 2017; Marcus et al., 2013; } \\
\text { Marcus et al., 2016) }\end{array}$ \\
\hline $\begin{array}{l}\text { Percentage of participants engaged in aerobic } \\
\text { activity }\end{array}$ & (Hovell et al., 2008) \\
\hline
\end{tabular}




\begin{tabular}{|c|c|}
\hline $\begin{array}{l}\text { PA in hours of moderate or greater activity } \\
\text { per week }\end{array}$ & $\begin{array}{l}\text { (Khare et al., 2014; Pekmezi et al., 2009; } \\
\text { Poston et al., 2001) }\end{array}$ \\
\hline Change in kilocalories as a measure of PA & (Poston et al., 2001; Rosal et al., 2005) \\
\hline Mean number of participant steps & $\begin{array}{l}\text { (King et al., 2013; Koniak-Griffin et al., 2015; } \\
\text { Vincent, 2009) }\end{array}$ \\
\hline
\end{tabular}

PA: physical activity MET: metabolic equivalent MVPA: moderate and vigorous PA 


\section{References}

Arredondo, E. M., Elder, J. P., Haughton, J., Slymen, D. J., Sallis, J. F., Perez, L. G., Serrano, N., Parra, M. T., Valdivia, R., \& Ayala, G. X. (2017). Fe en Acción: Promoting physical activity among churchgoing Latinas. American Journal of Public Health, 107(7), 11091115. https://doi.org/10.2105/AJPH.2017.303785

Ayala, G. X., Vaz, L., Earp, J. A., Elder, J. P., \& Cherrington, A. (2010). Outcome effectiveness of the lay health advisor model among Latinos in the United States: An examination by role. Health Education Research, 25(5), 815-840. https://doi.org/10.1093/her/cyq035

Babamoto, K. S., Sey, K. A., Camilleri, A. J., Karlan, V. J., Catalasan, J., \& Morisky, D. E. (2009). Improving diabetes care and health measures among Hispanics using community health workers: Results from a randomized controlled trial. Health Education \& Behavior, 36(1), 113-126. https://doi.org/10.1177/1090198108325911

Bell, J. A., Hamer, M., Batty, G. D., Singh-Manoux, A., Sabia, S., \& Kivimaki, M. (2014). Combined effect of physical activity and leisure time sitting on long-term risk of incident obesity and metabolic risk factor clustering. Diabetologia, 57(10), 2048-2056. https://doi.org/10.1007/s00125-014-3323-8

Brown, A. (2014, February 26). The U.S. Hispanic population has increased sixfold since 1970. http://www.pewresearch.org/fact-tank/2014/02/26/the-u-s-hispanic-population-hasincreased-sixfold-since-1970/

Bullard, K. M., Cowie, C. C., Lessem, S. E., Saydah, S. H., Menke, A., Geiss, L. S., Orchard, T. J., Rolka, D. B., \& Imperatore, G. (2018). Prevalence of diagnosed diabetes in adults by diabetes type - United States, 2016. MMWR. Morbidity and Mortality Weekly Report, 67(12), 359-361. https://doi.org/10.15585/mmwr.mm6712a2 
Caballero, A. E. (2005). Diabetes in the Hispanic or Latino population: Genes, environment, culture, and more. Current Diabetes Reports, 5(3), 217-225. https://doi.org/10.1007/s11892-005-0012-5

Centers for Disease Control and Prevention. (2014). Physical activity - Facts about physical activity. http://www.cdc.gov/physicalactivity/data/facts/htm

Centers for Disease Control and Prevention. (2018). Overweight and obesity - Causes and consequences. http://www.cdc.gov/obesity/adult/causes/index.html

Cerin, E., Cain, K. L., Oyeyemi, A. L., Owen, N., Conway, T. L., Cochrane, T., VAN Dyck, D., Schipperijn, J., Mitáš, J., Toftager, M., Aguinaga-Ontoso, I., \& Sallis, J. F. (2016). Correlates of agreement between accelerometry and self-reported physical activity. Medicine and Science in Sports and Exercise, 48(6), 1075-1084. https://doi.org/10.1249/MSS.0000000000000870

Coleman, K. J., Farrell, M. A., Rocha, D. A., Hayashi, T., Hernandez, M., Wolf, J., \& Lindsay, S. (2012). Readiness to be physically active and self-reported physical activity in lowincome Latinas, California WISEWOMAN, 2006-2007. Preventing Chronic Disease, 9 , E87.

Conn, V. S., Phillips, L. J., Ruppar, T. M., \& Chase, J. A. (2012). Physical activity interventions with healthy minority adults: Meta-analysis of behavior and health outcomes. Journal of Health Care for the Poor and Underserved, 23(1), 59-80. https://doi.org/10.1353/hpu.2012.0032 
Craig, C. L., Marshall, A. L., Sjöström, M., Bauman, A. E., Booth, M. L., Ainsworth, B. E., Pratt, M., Ekelund, U., Yngve, A., Sallis, J. F., \& Oja, P. (2003). International physical activity questionnaire: 12-country reliability and validity. Medicine and Science in Sports and Exercise, 35(8), 1381-1395. https://doi.org/10.1249/01.MSS.0000078924.61453.FB

Duggan, C., Carosso, E., Mariscal, N., Islas, I., Ibarra, G., Holte, S., Copeland, W., Linde, S., \& Thompson, B. (2014). Diabetes prevention in Hispanics: Report from a randomized controlled trial. Preventing Chronic Disease, 11, E28. https://doi.org/10.5888/pcd11.130119

Eakin, E. G., Bull, S. S., Riley, K. M., Reeves, M. M., McLaughlin, P., \& Gutierrez, S. (2007). Resources for health: A primary-care-based diet and physical activity intervention targeting urban Latinos with multiple chronic conditions. Health Psychology, 26(4), 392400. https://doi.org/10.1037/0278-6133.26.4.392

Gabrys, L., Sperzel, S., Bernhoerster, M., Banzer, W., \& Vogt, L. (2017). Real-time visual activity feedback for physical activity improvement in breast and colon cancer patients. Research in Sports Medicine, 25(1), 1-10. https://doi.org/10.1080/15438627.2016.1258639

Hawkins, M., Hosker, M., Marcus, B. H., Rosal, M. C., Braun, B., Stanek, E. J., 3rd, Markenson, G., \& Chasan-Taber, L. (2015). A pregnancy lifestyle intervention to prevent gestational diabetes risk factors in overweight Hispanic women: A feasibility randomized controlled trial. Diabetic Medicine, 32(1), 1080-115. https://doi.org/10.1111/dme.12601 
Hovell, M. F., Mulvihill, M. M., Buono, M. J., Liles, S., Schade, D. H., Washington, T. A., Manzano, R., \& Sallis, J. F. (2008). Culturally tailored aerobic exercise intervention for low-income Latinas. American Journal of Health Promotion, 22(3), 155-163. https://doi.org/10.4278/ajhp.22.3.155

Ickes, M. J., \& Sharma, M. (2012). A systematic review of physical activity interventions in Hispanic adults. Journal of Environmental and Public Health, 2012, 15. https://doi.org/10.1155/2012/156435

Keller, C., \& Fleury, J. (2006). Factors related to physical activity in Hispanic women. Journal of Cardiovascular Nursing, 21(2), 142-145.

Khare, M. M., Cursio, J. F., Locklin, C. A., Bates, N. J., \& Loo, R. K. (2014). Lifestyle intervention and cardiovascular disease risk reduction in low-income Hispanic immigrant women participating in the Illinois WISEWOMAN program. Journal of Community Health, 39(4), 737-746. https://doi.org/10.1007/s10900-014-9820-3

King, A. C., Bickmore, T. W., Campero, M. I., Pruitt, L. A., \& Yin, J. L. (2013). Employing virtual advisors in preventive care for underserved communities: Results from the COMPASS study. Journal of Health Communication, 18(12), 1449-1464. https://doi.org/10.1080/10810730.2013.798374

Kolt, G. S., Schofield, G. M., Kerse, N., Garrett, N., Ashton, T., \& Patel, A. (2012). Healthy Steps trial: Pedometer-based advice and physical activity for low-active older adults. The Annals of Family Medicine, 10(3), 206-212. https://doi.org/10.1370/afm.1345 
Koniak-Griffin, D., Brecht, M. L., Takayanagi, S., Villegas, J., Melendrez, M., \& Balcazar, H. (2015). A community health worker-led lifestyle behavior intervention for Latina (Hispanic) women: Feasibility and outcomes of a randomized controlled trial. International Journal of Nursing Studies, 52(1), 75-87. https://doi.org/10.1016/j.ijnurstu.2014.09.005

Kwon, S., Wang, M., \& Hawkins, M. (2016). Association between self-reported physical activity and obesity among White, Black, Hispanic, and Asian Americans: 2007 and 2009 BRFSS. Ethnicity \& Disease, 23(2), 129-135.

Marcus, B. H., Dunsiger, S. I., Pekmezi, D. W., Larsen, B. A., Bock, B. C., Gans, K. M., Marquez, B., Morrow, K. M., \& Tilkemeier, P. (2013). The Seamos Saludables study: A randomized controlled physical activity trial of Latinas. American Journal of Preventive Medicine, 45(5), 598-605. https://doi.org/10.1016/j.amepre.2013.07.006

Marcus, B. H., Hartman, S. J., Larsen, B. A., Pekmezi, D., Dunsiger, S. I., Linke, S., Marquez, B., Gans, K. M., Bock, B. C., Mendoza-Vasconez, A. S., Noble, M. L., \& Rojas, C. (2016). Pasos Hacia La Salud: A randomized controlled trial of an internet-delivered physical activity intervention for Latinas. The International Journal of Behavioral Nutrition and Physical Activity, 13, 62. https://doi.org/10.1186/s12966-016-0385-7

Marquez, D. X., Neighbors, C. J., \& Bustamante, E. E. (2010). Leisure time and occupational physical activity among racial or ethnic minorities. Medicine and Science in Sports and Exercise, 42(6), 1086-1093. https://doi.org/10.1249/MSS.0b013e3181c5ec05 
Marshall, S. J., Nicaise, V., Ji, M., Huerta, C., Haubenstricker, J., Levy, S. S., Ainsworth, B., \& Elder, J. E. (2013). Using step cadence goals to increase moderate-to-vigorous-intensity physical activity. Medicine and Science in Sports and Exercise, 45(3), 592-602. https://doi.org/10.1249/MSS.0b013e318277a586

Martinez, S. M., Ainsworth, B. E., \& Elder, J. P. (2008). A review of physical activity measures used among US Latinos: Guidelines for developing culturally appropriate measures. Annals of Behavioral Medicine, 36(2), 195-207. https://doi.org/10.1007/s12160-008-9063-6

Martinez, S. M., Arredondo, E. M., \& Roesch, S. (2013). Physical activity promotion among churchgoing Latinas in San Diego, California: Does neighborhood cohesion matter? Journal of Health Psychology, 18(10), 1319-1329. https://doi.org/10.1177/1359105312462433

McEwen, M. M., Pasvogel, A., Murdaugh, C., \& Hepworth, J. (2017). Effects of a family-based diabetes intervention on behavioral and biological outcomes for Mexican American adults. Diabetes Educator, 43(3), 272-285. https://doi.org/10.1177/0145721717706031

Mier, N., Medina, A. A., \& Ory, M. G. (2007). Mexican Americans with type 2 diabetes: Perspectives on definitions, motivators, and programs of physical activity. Preventing Chronic Disease, 4(2), A24.

Mier, N., Ory, M. G., \& Medina, A. A. (2010). Anatomy of culturally sensitive interventions promoting nutrition and exercise in Hispanics: A critical examination of existing literature. Health Promotion Practice, 11(4), 541-554. https://doi.org/10.1177/1524839908328991 
Mitchell, D. C., Andrews, T., \& Schenker, M. B. (2015). Pasos Saludables: A pilot randomized intervention study to reduce obesity in an immigrant farmworker population. Journal of Occupational and Environmental Medicine, 57(10), 1039-1046.

https://doi.org/10.1097/jom.0000000000000535

Moher, D., Liberati, A., Tetzlaff, J., Altman, D. G., \& and the PRISMA Group. (2009). Preferred reporting items for systematic reviews and meta-analyses: The PRISMA statement. Annals of Internal Medicine, 151(4), 264-269. https://doi.org/10.7326/0003-4819-151-4-200908180-00135

National Institute of Diabetes and Digestive and Kidney Diseases. (2017). Overweight and obesity statistics. https://www.niddk.nih.gov/health-information/healthstatistics/Pages/overweight-obesity-statistics.aspx\#c

Ogilvie, D., Foster, C. E., Rothnie, H., Cavill, N., Hamilton, V., Fitzsimons, C. F., Mutrie, N., \& Scottish Physical Activity Research Collaboration (2007). Interventions to promote walking: Systematic review. BMJ, 334(7605), 1204. https://doi.org/10.1136/bmj.39198.722720.BE

Pekmezi, D. W., Neighbors, C. J., Lee, C. S., Gans, K. M., Bock, B. C., Morrow, K. M., Marquez, B., Dunsiger, S., \& Marcus, B. H. (2009). A culturally adapted physical activity intervention for Latinas: A randomized controlled trial. American Journal of Preventive Medicine, 37(6), 495-500. https://doi.org/10.1016/j.amepre.2009.08.023

Perez, A., Fleury, J., \& Keller, C. (2010). Review of intervention studies promoting physical activity in Hispanic women. Western Journal of Nursing Research, 32(3), 341-362. https://doi.org/10.1177/0193945909351300 
Poston, W. S., 2nd, Haddock, C. K., Olvera, N. E., Suminski, R. R., Reeves, R. S., Dunn, J. K., Hanis, C. L., \& Foreyt, J. P. (2001). Evaluation of a culturally appropriate intervention to increase physical activity. American Journal of Health Behavior, 25(4), 396-406. https://doi.org/10.5993/ajhb.25.4.5

Rhodes, S. D., Foley, K. L., Zometa, C. S., \& Bloom, F. R. (2007). Lay health advisor interventions among Hispanics/Latinos: A qualitative systematic review. American Journal of Preventive Medicine, 33(5), 418-427.

https://doi.org/10.1016/j.amepre.2007.07.023

Rosal, M. C., Ockene, I. S., Restrepo, A., White, M. J., Borg, A., Olendzki, B., Scavron, J., Candib, L., Welch, G., \& Reed, G. (2011). Randomized trial of a literacy-sensitive, culturally tailored diabetes self-management intervention for low-income Latinos: Latinos en Control. Diabetes Care, 34(4), 838-844. https://doi.org/10.2337/dc10-1981

Rosal, M. C., Olendzki, B., Reed, G. W., Gumieniak, O., Scavron, J., \& Ockene, I. (2005). Diabetes self-management among low-income Spanish-speaking patients: A pilot study. Annals of Behavioral Medicine, 29(3), 225-235. https://doi.org/10.1207/s15324796abm2903_9

Rothschild, S. K., Martin, M. A., Swider, S. M., Tumialan Lynas, C. M., Janssen, I., Avery, E. F., \& Powell, L. H. (2014). Mexican American trial of community health workers: A randomized controlled trial of a community health worker intervention for Mexican Americans with type 2 diabetes mellitus. American Journal of Public Health, 104(8), 1540-1548. https://doi.org/10.2105/ajph.2013.301439 
Sangster, J., Church, J., Haas, M., Furber, S., \& Bauman, A. (2015). A comparison of the costeffectiveness of two pedometer-based telephone coaching programs for people with cardiac disease. Heart, Lung and Circulation, 24(5), 471-479.

https://doi.org/10.1016/j.hlc.2015.01.008

Sarma, S., Devlin, R. A., Gilliland, J., Campbell, M. K., \& Zaric, G. S. (2015). The effect of leisure-time physical activity on obesity, diabetes, high BP and heart disease among Canadians: Evidence from 2000/2001 to 2005/2006. Health Economics, 24(12), 15311547. https://doi.org/10.1002/hec.3106

Sharma, M. (2008). Physical activity interventions in Hispanic American girls and women. Obesity Reviews, 9(6), 560-571. https://doi.org/10.1111/j.1467-789X.2008.00501.x

Taylor, P., Lopez, M. H., Martinez, J., \& Velasco, G. (2012, April 4). When labels don't fit: Hispanics and their views of identity. http://www.pewhispanic.org/2012/04/04/whenlabels-dont-fit-hispanics-and-their-views-of-identity/

Turino, T. (2003). Nationalism and Latin American music: Selected case studies and theoretical considerations. Latin American Music Review / Revista de Musica Latinoamericana, 24(2), 169-209.

Vincent, D. (2009). Culturally tailored education to promote lifestyle change in Mexican Americans with type 2 diabetes. Journal of the American Academy of Nurse Practitioners, 21(9), 520-527. https://doi.org/10.1111/j.1745-7599.2009.00439.x

Wood, F. G. (2002). Ethnic differences in exercise among adults with diabetes. Western Journal of Nursing Research, 24(5), 502-515. https://doi.org/10.1177/019394502400446388 


\section{CHAPTER 3}

\section{METHODS}

\section{Research Design}

This pilot study utilized a pre-test/post-test, no control group design to examine a novel PA intervention. The Salud Paso por Paso (Health Step by Step) intervention consisted of 6 face-to-face sessions delivered once weekly for 6 weeks. Participants were divided into two groups of 10 and 11 participants, which allowed for physical distancing and effective delivery of the intervention. The study was conducted in a community with a large Hispanic population. The researcher is a member of the community and grew up in the geographical area served by the clinic that served as the recruitment site. The researcher is a native Spanish speaker and shares the same cultural background as the participants of the study.

\section{Sample}

The study sample consisted of Hispanic adults over 18 years of age that had been diagnosed with T2DM or prediabetes by a health care provider. Participants were recruited from the population served by Clínica Amistad, a free clinic located in a predominantly Hispanic community in Tucson, Arizona. Participants were required to obtain clearance from a health care provider (i.e. physician, nurse practitioner) to participate in this intervention study. Both English and Spanish speaking participants were eligible to participate.

Pilot studies typically involve smaller samples to determine efficacy of the intervention. Since anything greater than a $20 \%$ attrition rate is concerning for bias (Marcellus, 2004), the total sample size was 21 participants with 10 participants in one group and 11 participants in another group. The intervention sampling description follows the CONSORT statement guidelines by Moher, Schulz, \& Altman, (2001) (Appendix A); there were a total of 21 participants enrolled without randomization. 


\section{Recruitment}

Participants were recruited from Clínica Amistad, a free clinic located in Tucson, Arizona. Estimated time of recruitment to completion was prolonged during this study due to winter holidays. During this timeframe, many potential participants did not attend their clinic appointments due to being out of town. The researcher established a relationship with clinic leadership, clinic managers and medical directors through serving as a volunteer triage $\mathrm{RN}$ at Clínica Amistad. Participants were identified from existing clinic patients that had appointments to see a health care provider. Participants were recruited via a flyer, written in Spanish, placed in the clinic lobby and clinic exam rooms. The flyer asked potential participants to contact the researcher in person or by phone. The researcher also made general announcements in the waiting area of the clinic to increase awareness of the study. The researcher spoke with potential participants to outline purpose of the study, potential benefits and risks, and participant expectations. Written informed consent was obtained by fully explaining potential benefits and risks of the study for participants, participant rights and responsibilities, researcher rights and responsibilities, and compensation for participations. Enrolled study participants received compensation in the form of grocery store cards to promote study participation. Participants received a \$20 grocery store gift card for the enrollment and final intervention sessions and a $\$ 10$ grocery store gift card for sessions two to four (i.e. a total of $\$ 80$ USD). Retention of study participants was achieved through face-to-face meetings, telephone reminders, text-messaging reminders, and issuance of grocery store gift cards by the researcher. Alternative contact numbers were collected at baseline assessment study points and were rechecked during the study for accuracy (Eakin et al., 2007). Also, at least 3 contact attempts at various times of day and 
different days of the week were completed if participants missed a session; the attempted contacts consisted of 2 telephone calls and 1 text message. This intensive follow up and contact, along with grocery store gift cards provided to participants, improved participation and retention (Yancey, Ortega, \& Kumanyika, 2006).

One of the unexpected challenges of conducting this pilot study was the emergence of COVID-19 in March 2020. Necessary changes were made, such as ensuring all participants maintained appropriate physical distancing and wore masks during intervention and education sessions during the study to promote infection prevention. One of the challenges was to conduct group walks as this was the preferred method of participants. To facilitate social distancing during the walking portion of the intervention, the researcher required and monitored participants to maintain at least six feet of distance from other participants. This is an approach that has been used with other studies to comply with social distancing guidelines (Harkness et al, 2020). Also, while the participants received the educational portion of the intervention in small group settings, the researcher ensured that the participants were adhering to social distancing guidelines by setting chairs at least six feet apart and reminding participants to wear masks and to wash their hands with hand sanitizer provided during the meetings.

\section{Setting}

Clínica Amistad is a community partner that serves the priority population which live near the clinic. Clínica Amistad is a free health clinic that opened in 2003 in Tucson, Arizona; it serves Tucson's low-income uninsured community. Most clients of the clinic are unemployed or uninsured, with the majority having chronic medical conditions such as diabetes or hypertension. The mission of Clínica Amistad is to provide comprehensive health services, primary care, and free education to low-income people, who do not have health insurance or are marginalized, in 
an environment of friendship and support (Clínica Amistad, n.d.). Services provided include medical evaluations and treatments by a medical doctor, physician assistant, or a nurse practitioner; consultations for endocrinology, pulmonology, cardiology, and dermatology; medical and laboratory tests; massage therapy and other bodywork modalities such as Zero Balancing and Bowenwork; energy therapy such as Reike; health education about diet and exercise, and social services support. All of the services at the clinic are completely free to clients; however, donations are accepted. Also, the clinic is located in a complex that houses other services for community residents. As such, this neighborhood center serves as an entity where individuals form relationships and have overlapping and interacting social networks.

\section{Intervention}

The culturally-tailored physical activity (PA) intervention Salud Paso por Paso was informed from findings of a literature review of PA interventions with Hispanic adults in the United States (Loya, 2018). Effective strategies to promote PA in Hispanic adults include using interventions derived from theoretical frameworks, face-to-face delivery formats, and use of pedometers. The intervention incorporates elements of face-to-face intervention delivery and use of pedometers. The vast majority of Clinica Amistad's patients are of Mexican origin. There is evidence to suggest individuals of Mexican origin reported higher levels of work-related PA, but they were not necessarily the most active Hispanic group when measured by accelerometer (Arredondo et al., 2016). Moreover, there is evidence to suggest Hispanic adults such as Mexican-Americans do not meet recommended levels of PA (Tucker et al., 2011). Therefore, it was important to tailor PA interventions to specific aspects of the target population for this study. Culturally-tailored components of the PA intervention included delivering study materials in Spanish, simplified language of educational materials to engage participants with low literacy levels, providing the intervention in a community setting with which participants were familiar 
and comfortable, and the researcher sharing the same cultural background and primary language of participants.

Intervention development for Salud Paso por Paso was guided by Social Cognitive Theory (SCT) (Bandura, 2004). SCT includes health goals individuals set for themselves and concrete plans for achieving them; it also emphasizes building self-efficacy (Bandura, 2004).

The intervention consisted of providing culturally appropriate education to participants during an initial session and weekly meetings, and weekly reminder texts to engage in suggested PA and to attend the following week's meeting. There were six face-to-face meetings, with an initial education session of 60 minutes and 4 sessions of 45 minutes in duration every week. The intervention content was developed based on the American Diabetes Association (ADA) Standards of Medical Care in Diabetes (2017) and the recommended exercise plan for sedentary individuals by the ADA (2018). Furthermore, the educational content of the intervention was based on current American Heart Association (2014) guidelines for moderate PA.

\section{Intervention Activities}

Salud Paso por Paso was designed by the researcher in partnership with key stakeholders from Clinica Amistad. The intervention activities were presented to a focus group of stakeholders from the community for possible tailoring and for community members' input (Table 3.1). This group of stakeholders included nurses, physicians, and community members who are volunteers at Clínica Amistad. After receiving input from the Clínica Amistad community, the intervention was refined prior to beginning the pilot study. 
Table 3.1

Intervention activities

\begin{tabular}{|c|c|c|c|c|}
\hline Session & Duration & Content of session & Physical activity & $\begin{array}{l}\text { Data } \\
\text { collection }\end{array}$ \\
\hline 1 & 60 mins & $\begin{array}{cl}\text { - Intervention orientation procedure } \\
\text { Expectations of participants in } \\
\text { intervention } \\
\text { Validation of text messaging } \\
\text { capabilities with participants that } \\
\text { have cell phones to provide text } \\
\text { messages tailored to participants' } \\
\text { level of PA. } \\
\text { - Text messaging has shown promise as an } \\
\text { effective tool to aid in behavioral goal } \\
\text { setting and PA changes } \\
\text { - } \quad \text { Provision of pedometers to } \\
\text { participants } \\
\text { Instructions on use of } \\
\text { pedometer provided to } \\
\text { participants } \\
\text { Pedometer use is an effective } \\
\text { tool associated with } \\
\text { significant increases in PA } \\
\text { Provision of diaries to participants } \\
\text { Instructions on how to } \\
\text { complete diaries provided to } \\
\text { participants } \\
\text { Recommendations of PA based on } \\
\text { American Heart Association } \\
\text { guidelines (2014) } \\
\text { How to exercise safely }\end{array}$ & $\begin{array}{l}5 \text { minutes of slow } \\
\text { walking } \\
\text {-Instructions to walk } \\
\text { slowly for } 5 \text { minutes } \\
\text { for another } 4 \text { days } \\
\text { during the rest of the } \\
\text { week for } 5 \text { days total } \\
\text { of walking } \\
\text { - } \$ 20 \text { grocery store } \\
\text { gift card }\end{array}$ & $\begin{array}{l}\text {-SEEBS Scale } \\
\text {-7-Day PAR } \\
\text {-Demographic } \\
\text { data } \\
\text {-SASH }\end{array}$ \\
\hline 2 & 45 mins & $\begin{array}{l}\text { - } \quad \text { Group discussion on how } \\
\text { participants fared with their PA } \\
\text { during the last week } \\
\text { - Group discussion on potential } \\
\text { barriers participants might face } \\
\text { with PA } \\
\text { - How to overcome potential } \\
\text { barriers }\end{array}$ & $\begin{array}{l}5 \text { minutes of slow } \\
\text { walking } \\
5 \text { minutes of brisk } \\
\text { walking } \\
\text { - Instructions to } \\
\text { walk slowly for } 5 \\
\text { minutes and to walk } \\
\text { briskly for } 5 \text { minutes } \\
\text { for another } 4 \text { days } \\
\text { during the rest of the } \\
\text { week for } 5 \text { days total } \\
\text { of walking } \\
\text {-- } \$ 10 \text { grocery store } \\
\text { gift card }\end{array}$ & $\begin{array}{l}\text {-7 Day PAR } \\
\text {-Step count } \\
\text { from } \\
\text { pedometer }\end{array}$ \\
\hline 3 & 45 mins & $\begin{array}{l}\text { - Group discussion on how } \\
\text { participants fared with their PA } \\
\text { during the last week } \\
\text { - } \quad \text { Group discussion on motivators of } \\
\text { PA for participants } \\
\text { - How to stay motivated with PA }\end{array}$ & $\begin{array}{l}5 \text { minutes of slow } \\
\text { walking } \\
10 \text { minutes of brisk } \\
\text { walking } \\
\text { - Instructions to } \\
\text { walk slowly for } 5 \\
\text { minutes and to walk }\end{array}$ & $\begin{array}{l}\text {-7 Day PAR } \\
\text {-Step count } \\
\text { from } \\
\text { pedometer }\end{array}$ \\
\hline
\end{tabular}




\begin{tabular}{|c|c|c|c|c|}
\hline & & & $\begin{array}{l}\text { briskly for } 10 \\
\text { minutes for another } \\
4 \text { days during the } \\
\text { rest of the week for } \\
5 \text { days total of } \\
\text { walking } \\
-\$ 10 \text { grocery store } \\
\text { gift card }\end{array}$ & \\
\hline 4 & 45 mins & 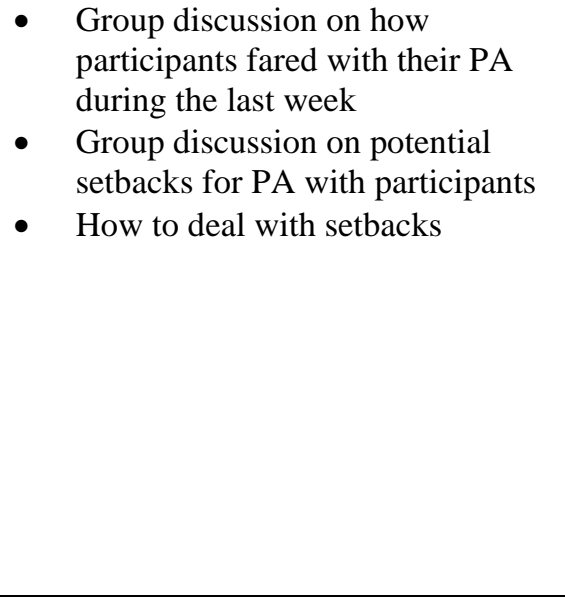 & $\begin{array}{l}5 \text { minutes of slow } \\
\text { walking } \\
15 \text { minutes of brisk } \\
\text { walking } \\
\text { - Instructions to } \\
\text { walk slowly for } 5 \\
\text { minutes and to walk } \\
\text { briskly for } 15 \\
\text { minutes for another } \\
4 \text { days during the } \\
\text { rest of the week for } \\
5 \text { days total of } \\
\text { walking } \\
-\$ 10 \text { grocery store } \\
\text { gift card }\end{array}$ & $\begin{array}{l}\text {-7 Day PAR } \\
\text {-Step count } \\
\text { from } \\
\text { pedometer }\end{array}$ \\
\hline 5 & 45 mins & $\begin{array}{l}\text { Group discussion on how } \\
\text { participants fared with their PA } \\
\text { during the last week } \\
\text { - Group discussion on how to } \\
\text { capitalize on support from } \\
\text { family/social network to engage in } \\
\text { PA with participants } \\
\text { - How to engage family/friends in } \\
\text { PA activity }\end{array}$ & $\begin{array}{l}5 \text { minutes of slow } \\
\text { walking } \\
20 \text { minutes of brisk } \\
\text { walking } \\
\text { - Instructions to } \\
\text { walk slowly for } 5 \\
\text { minutes and to walk } \\
\text { briskly for } 20 \\
\text { minutes for another } \\
4 \text { days during the } \\
\text { rest of the week for } \\
5 \text { days total of } \\
\text { walking } \\
-\$ 10 \text { grocery store } \\
\text { gift card }\end{array}$ & $\begin{array}{l}\text {-7 Day PAR } \\
\text {-Step count } \\
\text { from } \\
\text { pedometer }\end{array}$ \\
\hline 6 & $60 \mathrm{~min}$ & $\begin{array}{l}\text { Group discussion on how } \\
\text { participants fared with their PA } \\
\text { during the last week } \\
\text { - Group discussion on how } \\
\text { intervention program has helped } \\
\text { participants } \\
\text { - How to maintain exercise routine } \\
\text { for long term success }\end{array}$ & $\begin{array}{l}5 \text { minutes of slow } \\
\text { walking } \\
25 \text { minutes of brisk } \\
\text { walking } \\
\text { - Instructions to } \\
\text { walk slowly for } 5 \\
\text { minutes and to walk } \\
\text { briskly for } 25 \\
\text { minutes for another } \\
4 \text { days during the } \\
\text { rest of the week for } \\
5 \text { days total of } \\
\text { walking } \\
-\$ 20 \text { grocery store } \\
\text { gift card }\end{array}$ & $\begin{array}{l}\text {-SEEBS } \\
\text {-7 Day PAR } \\
\text {-Step count } \\
\text { from } \\
\text { pedometer }\end{array}$ \\
\hline
\end{tabular}


This intervention was designed to gradually increase the participants' quantity of PA. Since inclusion criteria included a diagnosis of T2DM, it was important to minimize risk of adverse events for participants. Also, many of the participants did not engage in leisure-time PA and engaged in sedentary behavior. Current recommendations for individuals with T2DM suggest starting exercise slowly and to focus on increasing duration rather than intensity of workouts (National Center on Health, Physical Activity and Disability, 2021). All participants For purposes of this intervention, self-efficacy is affected by performance mastery, vicarious experiences, and persuasion. This incremental approach supported the individual's ability to set achievable goals and built participant self-efficacy. Performance mastery, which encompasses past and present performance, is affected by self-efficacy beliefs (Bandura \& Locke, 2003).

\section{Face-to-Face Intervention Session Content}

Face-to-face meeting sessions provided support to participants by reinforcing topics discussed in previous sessions clarifying any questions or concerns participants might have, encouragement to continue with the PA intervention, and opportunities to measure progress with PA intervention implementation (Table 1). All participants were required to wear masks during the educational sessions and were required to maintain a physical distance of at least 6 feet apart. The initial meeting served to orient participants to the expectations of the study, including special instructions during demographic data collection. The content of each weekly meeting built on the previous week's content; participants received weekly printed material to review during the session with the researcher and to use for future reference. Content of the initial session focused on welcoming participants to the study and providing positive reinforcement on beginning to perform PA. Subsequent weekly meeting content focused on progressive increase of PA to achieve a target goal of $10 \%$ increase in steps from baseline. The text messages were 
tailored weekly to the level of PA each participant had performed during the previous week; however, text messages were scripted to ensure that participants received consistent messages. The researcher recorded duration of the meeting, quantity of PA measured in steps per day, and a summary of topics discussed with the participants. The researcher reviewed the data weekly to address any discrepancies and validate consistent content delivery. Each participant had a folder where information collected during the weekly meetings was kept. The information collected included discussion of the previous week's performance in achieving PA goal and barriers to achieving goals.

\section{Measures}

Demographic and health information data collected for this study at baseline included: age, gender, ethnicity, country of origin, marital status, educational level, income level, years living in the United States, language spoken at home, presence of additional health conditions, and acculturation scores. Outcome measures for Salud Paso por Paso included pre- and postintervention self-efficacy scores using the Self-Efficacy for Exercise Behaviors Scale (SEEBs), weekly steps per week, weekly minutes of PA, and PA recall. All measures are seen in Appendix $\mathrm{B}$ and $\mathrm{C}$.

The Self-Efficacy for Exercise Behaviors Scale (SEEBS) was developed by Sallis, Pinski, Grossman, Patterson, and Nader (1988) to measure self-efficacy in specific situations. Additionally, Sallis et al. (1988) examined the psychometric qualities of the SEEBS, providing information on the internal consistence and the test-retest reliabilities of the factors. Moreover, evidence of concurrent criterion-related validity was provided by determining relationships between self-efficacy ratings and reported health behaviors. The scale was developed from two studies; in the first study, Sallis et al. (1988) determined the behavioral components of eating a 
low-sodium, low-fat diet and engaging in regular exercise. The responses from the first study were used to construct test items administered in the second study. In the second study, the participants were administered draft self-efficacy scales of both eating and exercise behaviors; the SEEBS originally consisted of 49 items. Sallis et al. (1988) determined the factor structure of each scale using principal-components analysis with varimax rotation. The solution for the SEEBS contained 11 factors, accounting for $69 \%$ of the variance, but only two factors had eigenvalues $>2.0$. The authors named these factors "resisting relapse" and "making time for exercise;" the factors had five and seven items respectively. These two factors had test-retest reliability values of 0.68 at $p<0.001$ and Cronbach's alpha values of 0.85 for resisting relapse and 0.83 for making time for exercise (Sallis et al, 1988). The criterion-related validity was assessed by correlating self-efficacy factor scores with diet and exercise habits reported by participants. Both exercise factors were significantly correlated with values of 0.32 for resisting relapse and 0.40 for making time for exercise at $p<0.001$. For the specificity of the self-efficacy ratings, Sallis et al. (1988) found that self-efficacy for exercise was strongly related to current exercise habits with a one-way ANOVA value of 19.55 for resisting relapse and a value of 32.30 for making time for exercise $(p<0.001)$ and one factor (making time for exercise) was related to exercise attempts $(7.39, p<0.01)$. For construct validity, the correlation between multidimensional health locus of control (MHLC) subscales and health behaviors and selfefficacy factors showed the resisting relapse $(0.29, p<0.001)$ and making time for exercise $(0.42, p<0.001)$ were significant with the MHLC internal subscale and making time for exercise was significant for the MHCL change subscale $(-0.18, p<0.01)$. The SEEBS has been translated into French, Chinese, and Spanish, showing its adaptability to different populations. The Spanish version of the SEEBS contains 15 items. This is the only version of the SEEBS used as all 
participants preferred Spanish as the language used on study materials. Each item is scored on a Likert scale from 1 to 4 , with a score of 8 if the item does not apply (Appendix B). Participants completed this questionnaire during the initial and last sessions.

The Short Acculturation Scale for Hispanics (SASH) is a 12-item scale that provides information regarding acculturation (Marin, Sabogal, Marin, Otero-Sabogal, \& Perez-Stable, 1987). The SASH scale has been validated in a variety of samples, including low-income, immigrant Hispanics (Ellison, Jandorf, \& Duhamel, 2011). The $\alpha$ coefficient for the 12 common items of the SASH was 0.92 , establishing reliability. Validity analyses showed correlations of generation (0.65), length of residence (0.70), self-evaluation (0.76), acculturative index (0.83), and age of arrival (-0.69) at a significant level $(p<0.001)$ (Marin et al., 1987). Participants completed this measure during the initial session only.

Using pedometers to measure PA through counting steps has been shown as a valid and reliable method. There is evidence that shows that pedometers have concordance of activity measure between pedometers and accelerometers (median $r=0.86)$ and pedometers and direct observation (median $r=0.82$ ) (Tudor-Locke, Williams, Reis, \& Pluto, 2002). In terms of reliability and validity, one study showed validity and reliability of two Omron pedometer models through absolute percent error of each model (Holbrook, Barreira, \& Kang, 2009). Additionally, coefficient of variation $(\mathrm{CoV})$ values across slow, moderate, and very brisk walking speed for one pedometer model was $1.3 \%, 1.2 \%$, and $1.1 \%$ respectively; the CoV for the second model was $3.3 \%, 2.8 \%$ and $2.8 \%$ respectively. The coefficient of variation can be interpreted as the imprecision of an instrument (Whitley, Yong, \& Rasinen, 2015). Participants were instructed to wear the pedometer daily and record information on a log. These logs were 
collected during the weekly meetings. Participants had the opportunity to ask any questions and provide feedback on the use of pedometers.

The 7-Day Physical Activity Recall (7-Day PAR) Questionnaire by Sallis et al. (1985) is a semi-structured interview where the participant estimates time spent in PA, strength, and flexibility activities for the 7 days prior to the interview to determine duration and intensity of PA (Appendix C). This questionnaire has been validated for use in both English and Spanishspeaking populations (Zuazagoitia et al., 2014). The 7-Day PAR measured concurrent validity through Pearson product moment correlations between the phone PAR and accelerometer for total minutes per week of PA. The Pearson product moment correlations were $\mathrm{r}=0.43$, and $\mathrm{r}=$ 0.31 for moderate, $r=0.39$ for hard, and $r=0.78$ for very hard intensity activities. Pearson correlations between the in-person PAR and accelerometer for total minutes per week of PA were $r=0.41$, and $r=0.33$ for moderate, $r=0.43$ for hard, and $r=0.74$ for very hard intensity activities (Hayden-Wade, Coleman, Sallis, \& Armstrong, 2003). Participants completed this measure during the initial and last sessions.

\section{Procedures}

Signed informed consent from every participant was secured by the researcher; this ensured that participants fully understood their rights as human subjects involved in research. The participants were asked to read the informed consent form in their preferred language, or the researcher read it to the participants in their preferred language. All of the participants asked the researcher to read the consent aloud to them. The consent form provided the participant with information regarding their rights as a human subject, purpose of the study, and potential risks and benefits of the study. Also, by signing the informed consent form, the participant agreed to participate in the study. Each participant also received specific information regarding their 
choice to not participate in the study and the ability to withdraw from the study at any time without penalty. The researcher also signed the consent form in the indicated spaces. A copy of the competed consent form was provided to the participant and the original signed copy was placed in the participant's file.

Each participant had a unique identifying number (ID) that was utilized on all study materials for the duration of the study. The ID number was written on all data forms collected from the participant. The researcher has a master list of the participant names and their corresponding IDs in a secure location only accessible to the researcher to protect participant confidentiality. Furthermore, participants' name or other identifying information was not written on data collection forms to ensure confidentiality. The data collection forms are kept in the participant's intervention folder in a locked cabinet separate from the completed consent forms; the consent forms are kept in a locked cabinet in the researcher's office.

During the initial meeting, in addition to the consent information, the researcher verified the participant's contact information and wrote it in the Participant Locator Form (Appendix D). The purpose of this form was to collect data to enable the researcher to reach the participants if there was a need to reschedule a missed appointment or to reach a contact in case of emergency. Study IDs were assigned in the following format for all participants in a sequential manner: JLPA_001, JLPA_002, etc. until all eligible participants receive a unique ID.

\section{Overview of Intervention}

The researcher was responsible for scheduling weekly meetings and for conducting the intervention during group meetings. The researcher telephoned or sent a text message to participants to remind them of their upcoming meeting. The initial and last sessions were 60 minutes in duration while the rest of the weekly meetings were 45 minutes in duration; with the 
first group of participants, all meetings took place at a meeting area near a park. Many of the participants requested this meeting site as they could exercise and was easily accessible to them. The second group of participants formed two separate subgroups based on their availability for the weekly sessions. The meetings with this group took place in a meeting room at the community center. The researcher met with participants in a group setting for the educational component. Additionally, the researcher collected PA information during these meetings and discussed previous week's PA performance and set the following weekly PA goal with the participants. The researcher instructed wait-list control participants to follow their regular routine and not change their PA habits as much as possible until contacted to begin the intervention. Objective measurement of PA was recorded with an Omron Alvita USB Pedometer (model HJ322U, Kyoto, Japan). During the first meeting with participants, they received the Omron pedometer and received a demonstration from the researcher on its functions, how to check the battery level, and how to wear it. After PA data collection from participants occurred, the researcher used data from the pedometer to reinforce PA concepts with participants and to log number of steps walked by participants.

\section{Data Collection and Analysis}

The Salud Paso por Paso study addressed the following research questions:

1. Is the Salud Paso por Paso intervention feasible and acceptable in a group of adults living with T2DM and receiving care at Clinica Amistad?

2. What is the impact of the Salud Paso por Paso intervention, from pre- to postintervention on number of steps and minutes of PA?

Participants completed the SASH and a demographic questionnaire, created for this study, at baseline. Participants completed the SEEBS questionnaire and 7-Day PAR questionnaire during 
the initial and last intervention sessions. Participants completed self-reported daily PA logs with step counts from pedometer use as well as minutes of PA weekly for six weeks; participants completed the PA logs at home and brought them to the weekly intervention meetings. The questionnaires were available in English and Spanish, depending on participant's language preference; however, only Spanish questionnaires were used. Descriptive statistics were used to describe the study sample. Selected demographic data was analyzed to determine correlations between demographic characteristics and steps and minutes of PA per week. Outcome measures of steps and minutes per week and self-efficacy scores were analyzed using paired t tests to determine differences from pre- to post-intervention.

Qualitative data, in the form of field notes, was documented during the recruitment phase and after each intervention session. The researcher documented field notes to assess the acceptability and feasibility of the intervention activities and of the data collection activities (see Field Notes Guide, Appendix E).

\section{Implementation}

This pilot study examined the feasibility, acceptability, and initial impact of Paso por Paso, a novel culturally-tailored and theory-driven PA intervention. The group level PA intervention was implemented with a group of Hispanic adults of Mexican origin $(n=21)$. Within an ecological perspective, interventions at the group level are designed to elicit change in the knowledge, attitudes, and practices regarding a health concern within members of a priority population (Guttmacher, Kelly, \& Ruiz-Janecko, 2010). In this project, the priority population were members of the community that received care at a free clinic, Clinica Amistad, that served as the recruitment site. The ultimate goal of this program of research is to enhance the diabetes health outcomes of Hispanic adults living with T2DM. 


\section{Evaluation of Project Feasibility and Intervention Impact}

Community-based participatory research (CBPR) principles allow participants to provide rich feedback on PA intervention development and implementation (Wieland et al., 2018). While the project has quantitative outcomes, it was also important to collect data on several aspects of the intervention. From a quantitative standpoint, collecting demographic data, steps per week and minutes of PA per week, and self-reported efficacy scores was important. Collecting qualitative data such as field notes and participant evaluation of the proposed intervention was equally important. Using CBRP principles to form partnerships with community stakeholders such as clinic staff and patients increased the likelihood of intervention acceptance and feasibility (Carter et al., 2019). One of the important data sources are field notes. The field notes guide, used in this study, was adapted from the work of Berry, McQuiston, Parrado, and Olmos-Muñiz (2013). The guide helped collect information about the participant, and observational notes regarding informant behavior. Additionally, this field notes guide included methodological notes, theoretical notes, and personal notes of observations that researcher made during intervention implementation and intervention sessions (Appendix E).

\section{Provision of Feedback to the Community}

Providing insights gleaned from the evaluation of this project to participants can assist in instigating and sustaining desired changes (Chen, Diaz, Lucas, \& Rosenthal, 2010). The primary outcome of this intervention was to increase PA in this sample of the population. When participants engage in PA, there should be a corresponding improvement in their perception of engaging in PA as well as an objective increase in the number of steps per day. Information learned from this intervention was shared with participants. The preliminary findings of Salud Paso por Paso were shared with these groups through meetings at the community center when 
participants were available. Meetings were conducted in Spanish as this was all participants' primary language. Participants received information on preliminary findings of intervention acceptability, feasibility, and effectiveness. Clinica Amistad staff and volunteers received feedback on study findings during clinic hours as they expressed this was the most convenient time for them. Feedback included preliminary study results, including feasibility and acceptability of the PA intervention. Participants and Clinica Amistad staff/volunteers verbalized understanding of feedback provided. The interpretation of study findings was achieved through statistical analyses of outcome data. Statistical analyses included descriptive statistics and dependent t-tests to examine the impact of the intervention. Feasibility and acceptability of the intervention was examined through participant feedback recorded by the researcher in the form of field notes. 


\section{References}

American Diabetes Association. (2017). Standards of medical care in diabetes - 2017.

http://professional.diabetes.org/sites/professional.diabetes.org/files/media/ dc_40_s1_final.pdf

American Heart Association. (2014). American Heart Association recommendations for physical activity in adults. http://www.heart.org/HEARTORG/GettingHealthy/

PhysicalActivity/FitnessBasics/American-Heart-Association-Recommendations-forPhysical-Activity-in-Adults_UCM_307976_Article.jsp

Arredondo, E. M., Sotres-Alvarez, D., Stoutenberg, M., Davis, S. M., Crespo, N. C., Carnethon, M. R., Castañeda, S. F., Isasi, C. R., Espinoza, R. A., Daviglus, M. L., Perez, L. G., \& Evenson, K. R. (2016). Physical activity levels in U.S. Latino/Hispanic adults: Results from the Hispanic Community Health Study/Study of Latinos. American Journal of Preventive Medicine, 50(4), 500-508. https://doi.org/10.1016/j.amepre.2015.08.029

Bandura, A. (2004). Health promotion by social cognitive means. Health Educucation \& Behavior, 31(2), 143-164. https://doi.org/10.1177/1090198104263660

Bandura, A., \& Locke, E. A. (2003). Negative self-efficacy and goal effects revisited. Journal of Applied Psychology, 88(1), 87-99. https://doi.org/10.1037/0021-9010.88.1.87

Berry, N. S., McQuiston, C., Parrado, E. A., \& Olmos-Muñiz, J. C. (2013). Appendix G: Field notes guide. In B. A., Israel, E. Eng, A. Schulz, \& E. Parker (Eds.), Methods for community-based participatory research for health (2nd ed., 619-621). San Francisco, CA: Josey-Bass. 
Carter, W. M., Morse, W. C., Brock, R. W., \& Struempler, B. (2019). Improving physical activity and outdoor recreation in rural Alabama through community coalitions. Preventing Chronic Disease, 16, E116. https://doi.org/10.5888/pcd16.190062

Chen, P. G., Diaz, N., Lucas, G., \& Rosenthal, M. S. (2010). Dissemination of results in community-based participatory research. American Journal of Preventive Medicine, 39(4), 372-378. https://doi.org/10.1016/j.amepre.2010.05.021

Clínica Amistad. (n.d.). Quienes somos. http://www.clínicaamistad.org/ espanol/quienes-somos/

Eakin, E. G., Bull, S. S., Riley, K., Reeves, M. M., Gutierrez, S., \& McLaughlin, P. (2007). Recruitment and retention of Latinos in a primary care-based physical activity and diet trial: The Resources for Health study. Health Education Resesearch, 22(3), 361-371. https://doi.org/10.1093/her/cyl095

Ellison, J., Jandorf, L., \& Duhamel, K. (2011). Assessment of the Short Acculturation Scale for Hispanics (SASH) among low-income, immigrant Hispanics. Journal of Cancer Education, 26(3), 478-483. https://doi.org/10.1007/s13187-011-0233-z

Guttmacher, S., Kelly, P. J., \& Ruiz-Janecko, Y. (2010). Community-based health interventions: Principles and applications. San Francisco, CA: Jossey-Bass.

Harkness, A., Gattamorta, K. A., Estrada, Y., Jimenez, D., Kanamori, M., Prado, G., \& BeharZusman, V. (2020). Latinx health disparities research during COVID-19: Challenges and innovations. Annals of Behavioral Medicine, 54(8), 544-547. https://doi.org/10.1093/abm/kaaa054 
Hayden-Wade, H. A., Coleman, K. J., Sallis, J. F., \& Armstrong, C. (2003). Validation of the telephone and in-person interview versions of the 7-day PAR. Medicine and Science in Sports and Exercise, 35(5), 801-809. https://doi.org/10.1249/01.mss.0000064941.43869.4e

Holbrook, E., Barreira, T., \& Kang, M. (2009). Validity and reliability of Omron pedometers for prescribed and self-paced walking. Medicine \& Science in Sports \& Exercise, 41(3), 670674. https://doi.org/10.1249/MSS.0b013e3181886095

Loya, J. C. (2018). Systematic review of physical activity interventions in Hispanic adults. Hispanic Health Care International, 16(4), 174-188. https://doi.org/10.1177/1540415318809427

Marcellus, L. (2004). Are we missing anything? Pursuing research on attrition. Canadian Journal of Nursing Research, 36(3), 82-98

Marin, G., Sabogal, F., Marin, B. V., Otero-Sabogal, R., \& Perez-Stable, E. J. (1987). Development of a short acculturation scale for Hispanics. Hispanic Journal of Behavioral Sciences, 9(2), 183-205.

Moher, D., Schulz, K. F., \& Altman, D. G. (2001). The CONSORT statement: Revised recommendations for improving the quality of reports of parallel-group randomised trials. The Lancet, 357(9263), 1191-1194. https://doi.org/10.1016/S0140-6736(00)04337-3

National Center on Health, Physical Activity and Disability. (2021). Diabetes: Cardiovascular training guidelines. https://www.nchpad.org/88/665/Diabetes

Sallis, J. F., Haskell, W. L., Wood, P. D., Fortmann, S. P., Rogers, T., Blair, S. N., \& Paffenbarger, R. (1985). Physical activity assessment methodology in the Five City Project. American Journal of Epidemiology, 121, 91-106. 
Sallis, J. F., Pinski, R. B., Grossman, R. M., Patterson, T. L., \& Nader, P. R. (1988). The development of self-efficacy scales for healthrelated diet and exercise behaviors. Health Education Research, 3(3), 283-292.

Stewart, A. L., King, A. C., \& Haskell, W. L. (1993). Endurance exercise and health-related quality of life in 50-65 year-old adults. The Gerontologist, 33(6), 782-789.

Tucker, J. M., Welk, G. J., \& Beyler, N. K. (2011). Physical activity in U.S.: Adults compliance with the Physical Activity Guidelines for Americans. American Journal of Preventive Medicine, 40(4), 454-461. https://doi.org/10.1016/j.amepre.2010.12.016

Tudor-Locke, C., Williams, J. E., Reis, J. P., \& Pluto, D. (2002). Utility of pedometers for assessing physical activity. Sports Medicine, 32(12), 795-808. https://doi.org/10.2165/00007256-200232120-00004

Turner, D. W. (2010). Qualitative interview design: A practical guide for novice investigators. The Qualitative Report, 15(3), 754-760.

Ware Jr., J. E., Kosinski, M., \& Keller, S. D. (1996). A 12-Item Short-Form Health Survey: Construction of scales and preliminary tests of reliability and validity. Medical Care, 34(3), 220-233.

Wieland, M. L., Hanza, M., Weis, J. A., Meiers, S. J., Patten, C. A., Clark, M. M., Sloan, J. A., Novotny, P. J., Njeru, J. W., Abbenyi, A., Levine, J. A., Goodson, M., Capetillo, G., Osman, A., Hared, A., Nigon, J. A., \& Sia, I. G. (2018). Healthy immigrant families: Randomized controlled trial of a family-based nutrition and physical activity intervention. American Journal of Health Promotion, 32(2), 473-484. https://doi.org/10.1177/0890117117733342 
Whitley, H. P., Yong, E. V., \& Rasinen, C. (2015). Selecting an A1C point-of-care instrument. Diabetes Spectrum, 28(3), 201-208. https://doi.org/10.2337/diaspect.28.3.201

Yancey, A. K., Ortega, A. N., \& Kumanyika, S. K. (2006). Effective recruitment and retention of minority research participants. Annual Review of Public Health, 27(1), 1-28. https://doi.org/10.1146/annurev.publhealth.27.021405.102113

Zuazagoitia, A., Montoya, I., Grandes, G., Arietaleanizbeascoa, M., Arce, V., Martinez, V., Sanchez, M, \& Sanchez, A. (2014). Reliability and validity of the 7-day Physical Activity Recall interview in a Spanish population. European Journal of Sport Science, 14(sup1), S361-S368. https://doi.org/10.1080/17461391.2012.705332 
Appendix A. Study flow chart of Hispanic adults in physical activity intervention

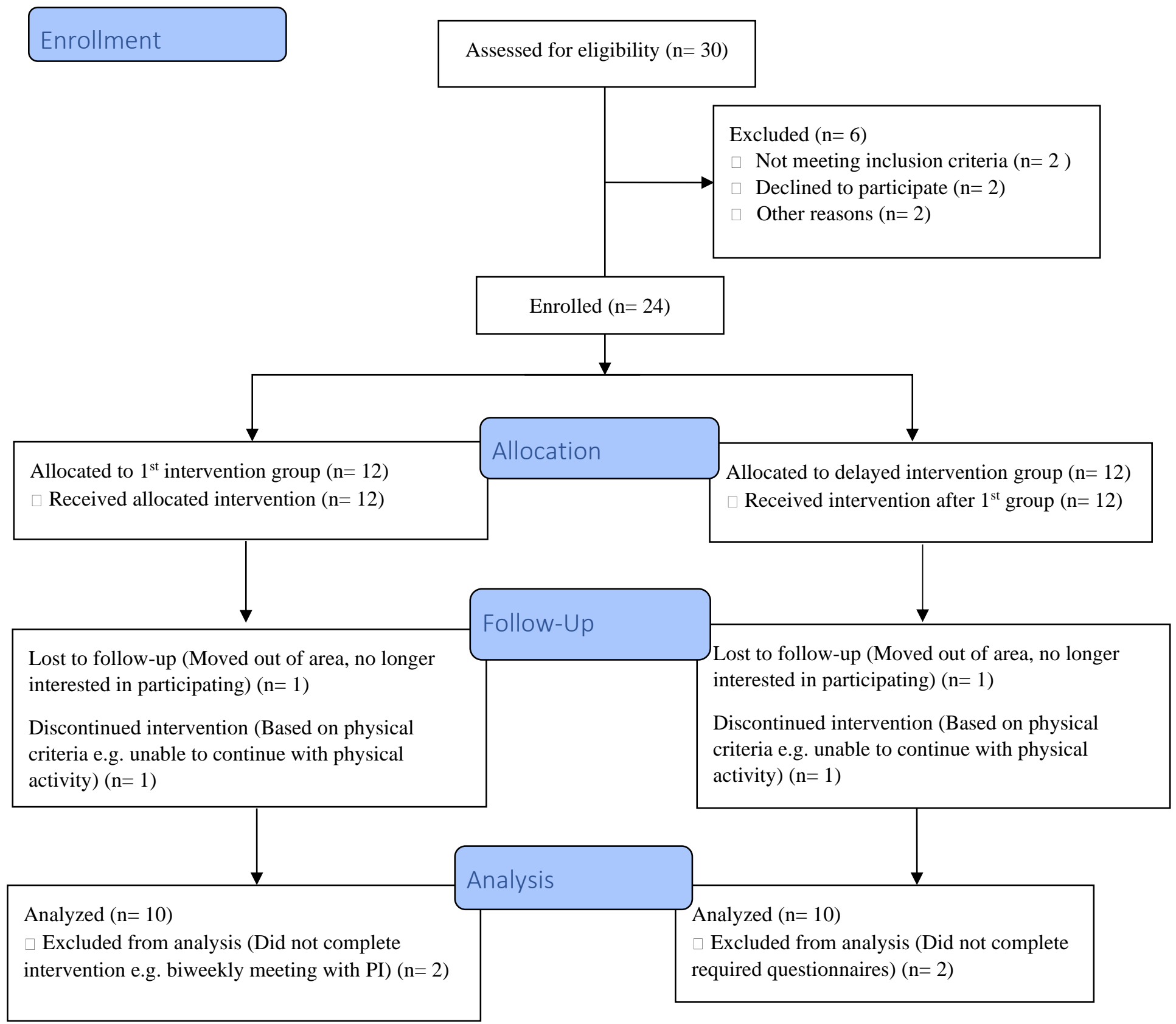

Figure 2. Study flow chart of Hispanic adults in physical activity intervention. Adapted from "The CONSORT Statement: Revised Recommendations for Improving The Quality of Reports of Parallel-Group Randomised Trials," by D. Moher, K. F. Schulz, \& D. G. Altman, 2001, The Lancet, 357(9263), p. 1193. Copyright 2001 by The Lancet. 
Appendix B. Self-efficacy for exercise behaviors scale

Nombre del participante:

\section{ESTUDIO DE CONFIANZA EN HACER EJERCICIOS}

Aqui hay una lista de cosas que uno pudiera hacer mientras está tratando de aumentar su nivel de ejercicios, o continuar un programa de ejercicios. Nos interesan los ejercicios como correr, nadar, caminar, andar en bicicleta, o asistir a clases de danza aerobica.

Por favor clasifique el nivel de confianza que tiene usted de que podria realmente motivarse a hacer estas cosas regularmente, por lo menos por seis meses.

¿QUE TAN SEGURO ESTA USTED DE PODER HACER ESTAS COSAS?

(Por favor circule un número por cada cosa.)

Estoy seguro Quizas si Estoy seguro No que no puedo puedaque si puedo aplica

16. Cambiar su horario de comer para hacer más conveniente el ejercicio

17. Levantarse más temprano para hacer ejercicios

18. Pedirle a sus vecinos o amigos de ir a caminar con usted regularmente

19. Planear el ejercicio dentro de su horario

1

20. Pedirle a sus amigos que hagan ejercicios 12

2

3

4

5 con usted.

21. Caminar en vez de manejar distancias cortas

22. Ir a una fiesta solo después de haber hecho sus ejercicios

23. Hacer ejercicios con su familia

24. Hacer ejercicios durante su hora de comida

25. Seguir con su programa de ejercicios después de un dia de trabajo largo y cansado.

26. Seguir con su programa de ejercicios aúnque tenga demasiadas exigencias en el trabajo.

27. Seguir con su program de ejercicios aúnque 1 tenga obligaciones sociales que le toman mucho tiempo. 
28. Hacer sus ejercicios aúnque se sienta deprimido 1

2

2

29. Empezar su programa de ejercicios de nuevo 1
después de una interrupción (como la navidad o lluvias).

30. Apartar tiempo para un programa de ejercicio, 1 por lo menos 30 minutos tres veces por semana
2

(1)

3

4 5

34
5

$3 \quad 4 \quad 5$

Figure 3. Self-efficacy for exercise behaviors scale. Adapted from "The development of self-efficacy scales for health-related diet and exercise behaviors," by J. F. Sallis, R. B. Pinski, R. M. Grossman, T. L. Patterson, \& P. R. Nader, 1988, Health Education Research, 3(3), p. 285. Copyright 1988 by Health Education Research. 
Appendix C. 7-Day Physical Activity Recall Questionnaire

7-Day Physical Activity Recall

SSN

PAR\#: 1223045667 Participant

Interviewer

Today is

Today's Date,

1. Were you employed in the last seven days?

0. No (Skip to Q\#4) 1. Yes

2. How many days of the last seven did you work?

days

3. How many total hours did you work in the last seven days?

4. What two days do you consider your weekend days?

WORKSHEET

hours last week

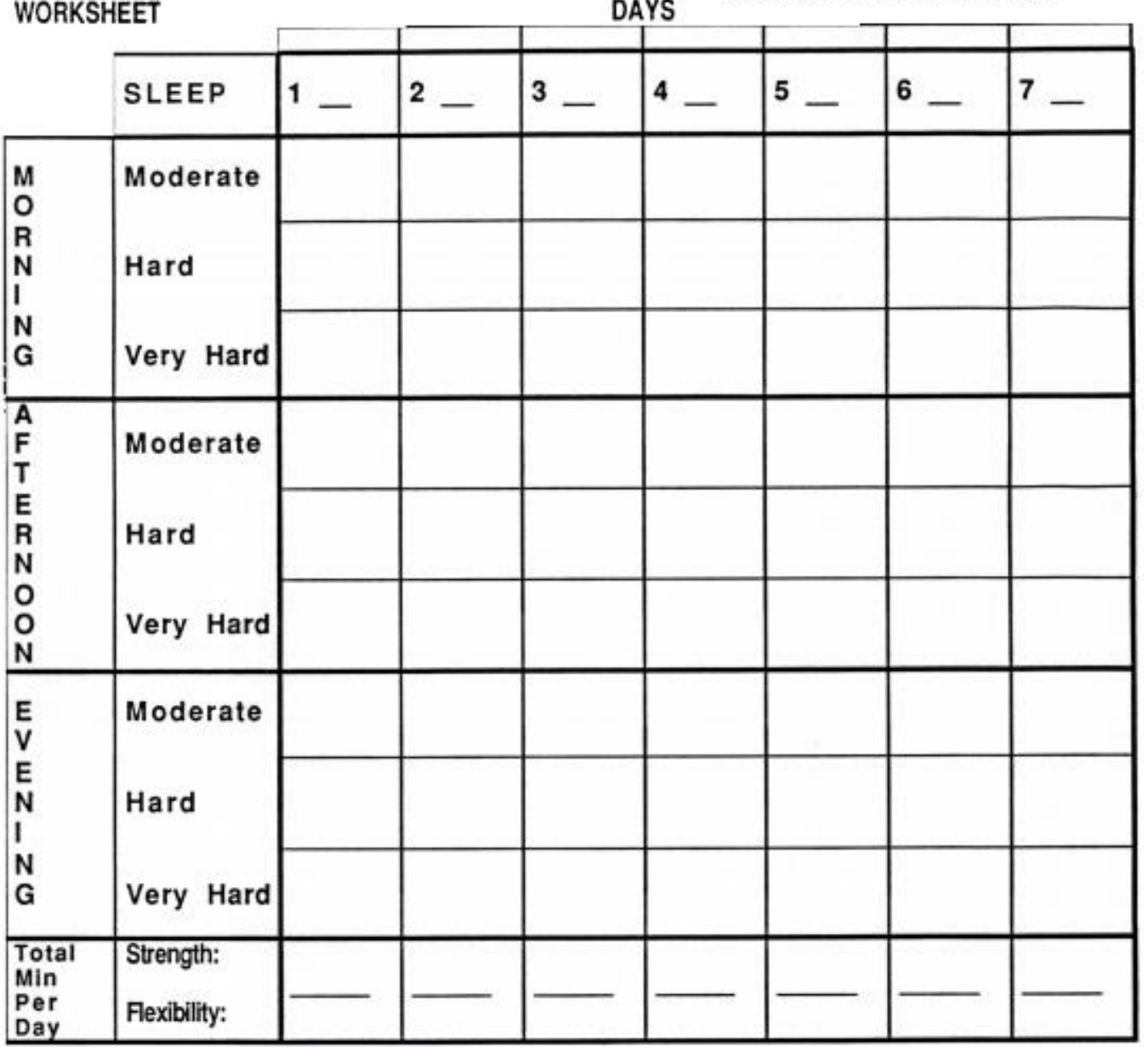

\begin{tabular}{|l|l|l|l}
\hline 4a. Compared to your physical activity over the past 3 months, & 6 . Do you think this was a valid PAR Interview?
\end{tabular} was last week's physical activity more, less, or about the same?

$\begin{array}{lll}\text { 1. More } & 2 \text {. Less } & 3 \text {. About the same }\end{array}$ 5. Were there any problems with the PAR interview?

$\begin{array}{ll}\text { 1. Yes } & 0 . \text { No }\end{array}$

11 NO, go to the beck and explain

0. No 1. Yes

If YES, go to the back and explain.

7. Were there any special circumstances concerning this PAR ?

0. No

1. Yes, If YES, what were they? (circle)

$\begin{array}{llll}\text { 1. Injury all week } & \text { 2. lliness all week } & \text { 3. Ilness part week }\end{array}$

4. Injury part week 5. Pregnancy 6. Other: 
5. Explain why there were problems with this PAR interview:

6. If PAR interview was not valid, why was it not valid?

7. Please list below any activities reported by the subject which you do not know how to classify.

8. Please provide any other comments you may have.

Sallis, J. F., Haskell, W. L., Wood , P. D., Fortmann, S. P., Rogers, T., Blair, S. N., and Paffenbarger, R. (1985). Physical activity assessment methodology in the Five City Project. American Journal of Epidemiology, 121, 91-106. 
Appendix D. Participant locator form

\section{Participant Locator Form}

\section{Personal Information}

Full Name:

\begin{tabular}{lll}
\hline Last & First & M.I.
\end{tabular}

Address:

\begin{tabular}{lll}
\hline City & State & ZIP Code
\end{tabular}

Home Phone:

Alternate Phone:

Email

Emergency Contact Information

Full Name:

\begin{tabular}{lll}
\hline Last & First & M.I.
\end{tabular}

Address:

Street Address

Apartment/Unit \#

City

State

ZIP Code

Primary Phone:

Alternate Phone:

Relationship: 
Appendix E

Field Notes Guide of Clínica Amistad Intervention

Participant code:

Recorder name:

Location:

Time :

Observational notes:

Methodological notes:

Theoretical notes:

Personal notes: 
Appendix F. Letter of Support

Clínica Amistad

101 W. Irvington Road

Building \#3

Tucson, AZ 85714

MU Human Subjects Research Protections Program/IRB:

Office of Research

University of Missouri

Columbia, MO 65211

To whom it may concern:

Please accept my commitment, and that of Clínica Amistad, to the proposed research project:

"Testing of a Culturally-Tailored Physical Activity Intervention in Hispanic Adults with Type 2 Diabetes Mellitus." This project aims to expand the knowledge base regarding physical activity interventions in Hispanic adults by using a culturally-tailored approach. This research approach exemplifies the overarching mission of Clínica Amistad and has the potential to answer important questions regarding specific aspects of physical activity intervention uptake with Hispanic adults with type 2 diabetes mellitus. Furthermore, findings from this project will promote understanding of better ways to implement physical activity research in Hispanic adults in the future.

Clínica Amistad is dedicated to creating and fostering an environment that improves and maintains the health and well-being of the most vulnerable members of our community. This project is congruent with Clínica Amistad's desire to improve the health of the community. Clínica Amistad is committed to this project. We see strong potential to build the knowledge and research base within this field while benefiting the residents of Tucson Arizona. Clínica Amistad is committed to working with Mr. Loya to increase awareness about the project in the community. Clínica Amistad will provide facility space to implement the intervention, as well as continuing to provide medical care and monitoring to participants.

We hope that your proposal receives a favorable review and we look forward to working with you on this foundational project.

Sincerely,

Dr. Rick Graap MD

Medical Director

Clínica Amistad 


\title{
CHAPTER 4
}

\section{RESULTS}

\section{Salud Paso por Paso: A culturally-tailored physical activity intervention with Hispanic}

\section{adults with type 2 diabetes mellitus}

\begin{abstract}
Limited information is available regarding culturally-tailored physical activity (PA) interventions for Hispanic adults with type 2 diabetes mellitus (T2DM). A community-partnered approach was used to examine a novel culturally-tailored PA intervention using a pre-post, no control group design. The intervention consisted of six weekly 45-minute sessions for participants to engage in PA led by the researcher. A total of 21 individuals participated in the study. The typical participant was a 53-year-old female (90\%) Hispanic adult living with T2DM with low acculturation. On average, before the intervention, the participants walked 10,285 (sd 14,779) steps per week with 43.4 (sd 68.1) minutes of PA per week. Despite implementation during the COVID-19 pandemic, the intervention was feasible and acceptable, and 19 (90.5\%) participants attended all intervention sessions. There were significant increases in steps per week $(p=0.007 ; d=1.03)$ and minutes of PA per week ( $p=0.000 ; d=1.62)$. Findings suggest that Salud Paso por Paso has promise as a strategy to enhance PA behaviors in the priority population. A randomized, controlled trial with a larger study sample is warranted to examine efficacy and impact on the diabetes health outcomes of Hispanic adults with T2DM.
\end{abstract}

Key words: Physical activity, Hispanic, T2DM, culturally-tailored intervention

This work was supported by a National Institute of Nursing Research-funded T32 Health Behavior Science Pre-Doctoral Fellowship

\section{Introduction}

Diabetes mellitus is a metabolic disease that affects approximately 34 million individuals in the United States (Centers for Disease Control and Prevention [CDC], 2019a). Among individuals living with diabetes, over $90 \%$ are classified as diabetes mellitus type 2 (T2DM) (CDC, 2019a). Prediabetes is a related condition where blood glucose levels are higher than normal, but not yet reaching T2DM levels (CDCa, 2020). Approximately 88 million adults in the United States have prediabetes: $84 \%$ of those with prediabetes are unaware of their status(CDCa, 2020). Risk factors for T2DM include prediabetes, overweight, age 45 years or older, immediate family members with T2DM, physical activity less than 3 times per week, history of gestational 
diabetes, and being African American, Hispanic/Latino American, Native American, or Alaska Native (CDCb, 2020). The Hispanic population in the United States is disproportionally affected by the burden of T2DM. Hispanic adults have a greater than 50\% risk for developing diabetes, than other U.S. adults (CDCc, 2019). Additionally, Hispanic individuals with T2DM have a higher incidence and prevalence of nephropathy, diabetic eye disease, and higher incidence of diabetes-related amputations (Aguayo-Mazzucato et al., 2019). Furthermore, older Hispanic adults incur higher costs for diabetes care than non-Hispanic whites (Glantz et al., 2019). The U.S. Hispanic population accounts for approximately $18 \%$ of the total U.S. population and it is growing at a rate higher than that of other groups (Noe-Bustamante et al., 2020).

The incidence and prevalence of T2DM is significant due to its close link to cardiovascular disease (CVD). Risk factors for CVD are prevalent among individuals with T2DM and include obesity, hypertension, dyslipidemia, and diabetic cardiomyopathy. Evidence suggests individuals with T2DM and CVD have an increased mortality risk (Branch et al., 2019). Another condition closely related to T2DM is metabolic syndrome, which is a constellation of abnormalities that include abdominal obesity, hypertension, dyslipidemia, and elevated blood glucose; the presence of metabolic syndrome significantly increases the risk of developing CVD (Punthakee et al., 2019). Physical activity (PA), along with diet and medication, is one of the cornerstones to enhance health outcomes for people living with T2DM. There is ample evidence to suggest that regular exercise helps to reduce weight and blood pressure, and improve dyslipidemia (Myers et al., 2019). Current PA guidelines state that for substantial health benefits, adults should engage in at least 150 minutes to 300 minutes a week of moderate-intensity, or 75 minutes to 150 minutes a week of vigorous-intensity aerobic PA (U.S. Department of Health and Human Services, 2021). 
Due to the rapid, and growing, rise in the number of Hispanic individuals in the United States, there is an obligation to promote diabetes care that incorporates elements of Hispanic culture and addresses the unique health care needs of this population. Effective strategies are needed to promote moderate intensity PA among Hispanic adults living with T2DM (Loya, 2018). Approaches such as starting with light intensity walking and breaking up time sitting with sanding show promise in improving T2DM outcomes (Duvivier et al., 2017). The Salud Paso por Paso (Health Step by Step) pilot intervention study, guided by Social Cognitive Theory (SCT) (Bandura, 1989, 2004), sought to enhance self-efficacy for PA and increase engagement in PA. A community-partnered approach was used to develop, and pilot test, the culturally tailored and linguistically appropriate (McCurley et al., 2017) group level intervention among Hispanic adults living with T2DM. The Salud Paso Por Paso study addressed the following research questions:

1. Is the Salud Paso por Paso intervention feasible and acceptable in a group of adults living with T2DM and receiving care at Clinica Amistad?

2. What is the impact of the Salud Paso por Paso intervention, from pre- to postintervention on number of steps and minutes of PA?

\section{Methods}

A need for effective strategies to enhance PA among Hispanic adults living with T2DM was identified by the Clinica Amistad (Friendship Clinic) community, located in the south side of Tucson, Arizona. Input for development of the novel PA intervention was provided by community members and staff from the clinic (i.e. medical staff, nurse volunteers, and a diabetic nurse educator). Intervention content was developed in accordance to established American Diabetes Association (2017) and American Heart Association (2014) guidelines for PA. To build 
trust in the community, the researcher served in a volunteer capacity as a triage nurse at Clinica Amistad for approximately prior to initiating any study activities. The researcher shared the cultural and linguistic background of the participants. As a native of Mexico and a fluent Spanish speaker, the researcher was in a unique position to build relationships with the community. Using a pre-test/post-test no control group design, this pilot study examined the feasibility, acceptability, and preliminary impact of Salud Paso por Paso, a PA intervention among Hispanic adults living with T2DM. PA outcomes measured included (1) total number of steps per week, (2) total minutes of PA per week per participant, (3) 7-day PA recall, and (4) selfefficacy for PA scores. Demographic and health characteristics, including acculturation scores, were collected to describe the study sample.

\section{Sample}

A convenience sample of Hispanic adults living with T2DM were recruited from Clinica Amistad, a free clinic which serves predominantly low-income individuals. Over $87 \%$ of clinic patients self-identified as Hispanic in 2019; the clinic serves a large number of Spanish speaking adults living with T2DM. Inclusion criteria were confirmed diagnosis of T2DM by a health care provider, ability to speak English or Spanish, previously sedentary. In addition, all participants received a health screening and exam and were cleared for study participation by a physician or nurse practitioner prior to participating in intervention sessions.

\section{Measures}

Demographic data and health information. Age in years, gender, ethnicity, birthplace, marital status, educational level, income level, and additional health conditions were obtained through self-report. Demographic data and health information were collected at the pre-intervention time point. 
Acculturation. Participants completed the Short Acculturation for Hispanics Scale (SASH) at the pre-intervention time point using a paper and pencil survey. The SASH measures changes that Hispanic individuals experience in values, norms, attitudes, and behaviors when exposed to mainstream cultural patterns of the United States (Marin et al., 1987).

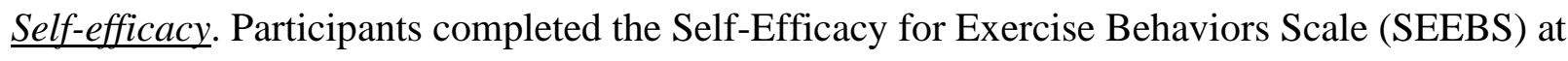
the pre-intervention time point and immediately post-intervention using a paper and pencil survey. The SEEBS scores was used to measure self-efficacy to exercise. The SEEBS questions are divided by subscales of "making time for exercise" and "sticking to it." Self-efficacy for participants was defined as the perception of the participant to engage in exercise behaviors. The SEEBS measures self-efficacy in a Likert scale from 1-4, with higher scores relating to higher self-efficacy (Sallis et al., 1988). Lower SEEBS scores signify that participants believe they cannot accomplish the behaviors; conversely, higher SEEBS scores signify participants' belief that they can accomplish the behaviors.

Exercise $\operatorname{logs}$ and PA recall. Frequency, number of steps, and number of minutes spent in PA were recorded by participants using paper and pencil logs. Participants received a pedometer and instructions on how and when to wear the pedometer. Participants were asked to record steps per day as measured by pedometer and minutes spent in PA and to bring the logs to each weekly session. Participants also received the 7-Day Physical Activity Recall (7-Day PAR) paper questionnaire during the first and last weeks of the intervention. The 7-Day PAR measures estimates of time spent in PA, strength, and flexibility activities for the 7 days prior to questionnaire completion (Sallis et al., 1985). Logs were reviewed weekly by the PI and collected at the end of each weekly session. 
Feasibility and acceptability. Feasibility was evaluated based on the ability to recruit participants, attendance at each weekly session, and completion of intervention components, which included daily logs documenting PA. Field notes were documented by the researcher, using a field notes guide (Appendix E), throughout the duration of the study to capture participants' perceptions of the feasibility and efficacy of the intervention. In addition to aspects related to feasibility and acceptability, field notes were also taken to determine any salient aspects of the intervention such as participant barriers and facilitators to engage in PA and complete intervention requirements.

\section{Procedure}

Approval for this study was obtained from the University of Missouri Institutional Review Board. Participants were recruited through flyers and generalized announcements made at the clinic waiting area. The researcher shared all aspects of the study with potential participants by meeting individually with them in a private area of the clinic. Written informed consent was obtained from participants prior to study participation. All data were collected using paper and pencil surveys. Table 1 provides an overview of the, Salud Paso por Paso activities.

\section{Table 4.1}

Salud Paso por Paso Intervention Activities by Week

\begin{tabular}{|c|c|c|c|}
\hline Session & Duration & Content of educational session & Physical activity \\
\hline 1 & 60 mins & $\begin{array}{l}\text {-Intervention orientation procedure: } \\
\text { participant responsibilities, materials provided } \\
\text { to participants (e.g. pedometers and paper } \\
\text { logs) } \\
\text {-Recommendations of PA based on American } \\
\text { Heart Association guidelines (2014), } \\
\text { including exercise safety }\end{array}$ & $\begin{array}{l}-5 \text { minutes of slow walking with } \\
\text { participants and researcher } \\
\text {-Participants to walk slowly for } 5 \\
\text { minutes for } 5 \text { days during this week } \\
-\$ 20 \text { gift card provided }\end{array}$ \\
\hline 2 & 45 ins & $\begin{array}{l}\text {-Group discussion on ability to engage and } \\
\text { maintain PA in last week; discussion on } \\
\text { potential barriers participants might face with } \\
\text { PA, and how to overcome potential barriers }\end{array}$ & $\begin{array}{l}-5 \text { minutes of slow walking, } 5 \\
\text { minutes of brisk walking } \\
\text { - Participants to walk slowly for } 5 \\
\text { minutes and to walk briskly for } 5 \\
\text { minutes for } 5 \text { days during this week } \\
-\$ 10 \text { gift card provided }\end{array}$ \\
\hline
\end{tabular}




\begin{tabular}{|l|l|l|l|}
\hline 3 & 45 ins & $\begin{array}{l}\text {-Group discussion on ability to engage and } \\
\text { maintain PA in last week; discussion on } \\
\text { motivators of PA for participants, and how to } \\
\text { stay motivated to engage in PA }\end{array}$ & $\begin{array}{l}\text {-5 minutes of slow walking, 10 } \\
\text { minutes of brisk walking } \\
\text { - Participants to walk slowly for 5 } \\
\text { minutes and to walk briskly for 10 } \\
\text { minutes for 5 days during this week } \\
\text { \$10 gift card provided }\end{array}$ \\
\hline 4 & 45 mins & $\begin{array}{l}\text { Group discussion on ability to engage and } \\
\text { maintain PA in last week; discussion on } \\
\text { potential setbacks to engage in PA and how to } \\
\text { deal with setbacks }\end{array}$ & $\begin{array}{l}-5 \text { minutes of slow walking, 15 } \\
\text { minutes of brisk walking } \\
\text {-Participants to walk slowly for 5 } \\
\text { minutes and to walk briskly for } 15 \\
\text { minutes for 5 days during this week } \\
\text { \$10 gift card provided }\end{array}$ \\
\hline 5 & 45 mins & $\begin{array}{l}\text { Group discussion on ability to engage and } \\
\text { maintain PA in last week; discussion on how } \\
\text { to capitalize on support from family/social } \\
\text { network to engage in PA and how to engage } \\
\text { family/friends in PA activity }\end{array}$ & $\begin{array}{l}\text {-5 minutes of slow walking, 20 } \\
\text { minutes of brisk walking } \\
- \text { Participants to walk slowly for 5 } \\
\text { minutes and to walk briskly for } 20 \\
\text { minutes for 5 days during this week } \\
\text { \$10 gift card provided }\end{array}$ \\
\hline 6 & 60 min & $\begin{array}{l}\text { Group discussion on ability to engage and } \\
\text { maintain PA in last week; discussion on how } \\
\text { intervention program has benefited } \\
\text { participants and how to sustain PA for long } \\
\text { term success }\end{array}$ & $\begin{array}{l}5 \text { minutes of slow walking } \\
25 \text { minutes of brisk walking } \\
\text { Participants to walk slowly for 5 } \\
\text { minutes and to walk briskly for } 25 \\
\text { minutes for 5 days during this week } \\
\text { \$20 gift card provided }\end{array}$ \\
\hline
\end{tabular}

\section{Data analysis}

Data were analyzed using statistical software IBM SPSS Statistics (Version 26) and screened for normality, missing data, and outliers. Ranges, means, and standard deviations for demographic data, self-efficacy scores, and PA frequency were calculated at baseline and at intervention completion. Paired-sample $t$ tests were performed to examine differences between pre- and post-intervention measures on self-efficacy, steps per week, and minutes of PA per week. Generalized estimating equations were performed on selected participant characteristics to discern any correlations with outcomes of steps and minutes of PA per week.

\section{Results}

A total of 21 Hispanic adults living with T2DM enrolled in the Salud Paso por Paso study. Two participants did not complete the intervention. One participant attended the first week of the intervention but withdrew from the study due to work schedule conflicts with intervention meeting times. Another participant withdrew from the study due to work schedule conflicts as 
well as the need to take care of a family member. Participants ranged in age from 30 to 75 years (mean 53, sd 11.8). The majority of participants were female (90\%), and most (76\%) were married. All participants, except one, identified Mexico as their country of origin. Most participants had an income level below $\$ 35,000$, with many participants reporting an income level less than $\$ 15,000$. Over half of participants had completed high school or less than a high school education. Participants had lived in the United States from 3 months to 35 years (mean 14.9, sd 11.25). While the researcher explicitly recruited individuals diagnosed with T2DM by a health care provider, some participants did not state they were diagnosed with any health condition on the demographic questionnaire. Over half of participants reported another chronic health condition in addition to T2DM or prediabetes. Participants had a mean SASH score of 1.53 (sd 0.744) at baseline, indicating a lesser acculturation level (Table 2).

Table 4.2

Salud Paso por Paso Participant Demographic and Health Characteristics

\begin{tabular}{|l|l|}
\hline Characteristics & Overall Percentage/(Mean/sd) $(n=21)$ \\
\hline Age & $53.0(11.807)$ \\
\hline Gender & \\
\hline Female & $90.5 \%$ \\
\hline Male & $9.5 \%$ \\
\hline Ethnicity & \\
\hline Mexican & $95.2 \%$ \\
\hline Dominican & $4.8 \%$ \\
\hline Marital status & \\
\hline Single/Divorced & $19.1 \%$ \\
\hline Married/Widowed & $76.2 \%$ \\
\hline No answer & $4.8 \%$ \\
\hline Yearly household income & \\
\hline$<\$ 14,999$ & $42.9 \%$ \\
\hline$\geq \$ 15,000-\$ 34,999$ & $19.1 \%$ \\
\hline$\geq \$ 35,000$ & $9.6 \%$ \\
\hline No answer & $28.6 \%$ \\
\hline Education level & \\
\hline $\begin{array}{l}\leq \text { High school } \\
\text { graduate }\end{array}$ & $33.4 \%$ \\
\hline
\end{tabular}




\begin{tabular}{|l|l|}
\hline High school graduate & $33.3 \%$ \\
\hline$\geq$ College graduate & $28.5 \%$ \\
\hline No answer & $4.8 \%$ \\
\hline Years living in the US & $14.94(11.25)$ \\
\hline Primary language at home & $100 \%$ \\
\hline Spanish & \\
\hline Other chronic health conditions & $57.1 \%$ \\
\hline Present (T2DM, prediabetes, HTN, other) & $42.9 \%$ \\
\hline $\begin{array}{l}\text { Not present/did not } \\
\text { answer }\end{array}$ & $1.53(0.744)$ \\
\hline SASH Score & \\
\hline
\end{tabular}

Self Efficacy for Exercise (SEEBS): 19 out of 21 (90\%) participants completed the pre- and postSEEBS questionnaire. Pre-intervention SEEBS mean scores for participants were 3.527 (sd 0.647) and post-intervention SEEBS mean scores were 3.602 (sd 0.420). Subscale scores for preand post- intervention were similar for "making time for exercise" (pre mean 3.650, sd 0.54, post mean 3.648, sd 0.392) and "sticking to it" (pre mean 3.450, sd 0.744, post mean 3.572, sd 0.458). A score of $>3.5$ suggests that all of the participants had high self-efficacy to engage in exercise prior to starting the intervention. All participants who enrolled in this study had high selfefficacy scores at baseline and there were no significant changes on self-efficacy total scores or subscale scores from pre- to post intervention.

PA steps and minutes per week: Steps per week and minutes of PA per week were recorded by participants during the intervention using a paper and pencil log. Two participants provided PA data for 1 week each and then withdrew from the study. 19 participants completed all recorded outcome measures of steps and minutes per week in their logs. Table 4 displays the results for paired samples $t$ test for each week compared to week 1 for both steps per week and minutes per week of PA. All weeks had significant changes in steps and minutes of PA from week one with the exception of week 5 for steps per week. The number of steps and minutes engaged in PA 
significantly increased from pre to post intervention (Table 4). Participants walked an average of almost 9,000 steps more $(p=0.007 ; d=1.03)$ and engaged in 74 minutes of PA per week more $(p=0.000 ; d=1.62)$ from week 1 to week 6.

\section{Table 4.3}

Salud Paso por Paso Differences in Number of Steps and Minutes of Exercise by Week from Pre to Post Intervention

\begin{tabular}{|l|l|l|l|l|l|l|}
\hline \multicolumn{1}{|c|}{$\begin{array}{l}\text { Week } \\
\text { comparison }\end{array}$} & \multicolumn{1}{|c|}{ Indicator } & \multicolumn{1}{|c|}{ Mean (SD) } & \multicolumn{1}{c|}{$\begin{array}{c}\text { 95\% CI } \\
\text { Lower }\end{array}$} & $\begin{array}{c}\text { 95\% CI } \\
\text { Upper }\end{array}$ & \multicolumn{1}{c|}{ t } & $p$-value \\
\hline $\begin{array}{l}\text { Week 1 to } \\
\text { Week 2 }\end{array}$ & Steps per week & $-10285.1(14779.7)$ & -17408.7 & -3161.5 & -3.033 & 0.007 \\
& $\begin{array}{l}\text { Minutes per } \\
\text { week }\end{array}$ & $-43.4(68.1)$ & -76.2 & -10.6 & -2.78 & 0.012 \\
\hline $\begin{array}{l}\text { Week 1 to } \\
\text { Week 3 }\end{array}$ & Steps per week & $-18474.5(25511.4)$ & -30770.6 & -6178.4 & -3.157 & 0.005 \\
& $\begin{array}{l}\text { Minutes per } \\
\text { week }\end{array}$ & $-50.8(51.7)$ & -75.8 & -25.9 & -4.284 & 0.000 \\
\hline $\begin{array}{l}\text { Week 1 to } \\
\text { Week 4 }\end{array}$ & Steps per week & $-18928.7(34756.2)$ & -35680.7 & -2176.8 & -2.374 & 0.029 \\
& $\begin{array}{l}\text { Minutes per } \\
\text { week }\end{array}$ & $-82.9(82.2)$ & -122.5 & -43.3 & -4.399 & 0.000 \\
\hline $\begin{array}{l}\text { Week 1 to } \\
\text { Week 5 }\end{array}$ & Steps per week & $-17102.6(26820.3)$ & -30029.6 & -4175.6 & -2.780 & 0.12 \\
& $\begin{array}{l}\text { Minutes per } \\
\text { week }\end{array}$ & $-102.2(156.5)$ & -177.6 & -26.8 & -2.848 & 0.011 \\
\hline $\begin{array}{l}\text { Week 1 to } \\
\text { Week 6 }\end{array}$ & Steps per week & $-19197.4(27719.6)$ & -32557.8 & -5837 & -3.019 & 0.007 \\
& $\begin{array}{l}\text { Minutes per } \\
\text { week }\end{array}$ & $-117.7(114.7)$ & -173.0 & -62.5 & -4.475 & 0.000 \\
\hline
\end{tabular}

\section{Relationships between steps/minutes of PA per week and selected demographic variables:}

Generalized estimating equations model for both steps and minutes to determine correlations with selected participant characteristics of age, years living in the United States, marital status, educational level, and presence of medical conditions (Table 5). There were no significant results 
for correlation estimates of steps per week. Correlation estimates of minutes of PA per week showed significant results for marital status, and absence of medical conditions. Single/divorced participants and those who did not disclose medical conditions engaged in average in more minutes of PA per week. Gender was not included in the analysis as $90 \%$ of the participants were female.

\section{Table 4.4}

Salud Paso por Paso Relationships Between Demographic Characteristics \& Steps/Minutes of PA Per Week

\begin{tabular}{|l|l|l|l|l|l|l|}
\hline Parameter & Indicator & B & Std. error & $\begin{array}{l}95 \% \text { CI } \\
\text { lower bound }\end{array}$ & $\begin{array}{l}95 \% \text { CI } \\
\text { upper bound }\end{array}$ & $\begin{array}{l}p- \\
\text { value }\end{array}$ \\
\hline Age & Steps per week & -417.03 & 543.88 & -1483.02 & 648.96 & 0.443 \\
& Minutes per week & 3.21 & 2.51 & -1.72 & 8.13 & 0.202 \\
\hline $\begin{array}{l}\text { Years living in } \\
\text { the US }\end{array}$ & Steps per week & -762.96 & 576.74 & -1893.35 & 367.44 & 0.186 \\
\hline $\begin{array}{l}\text { Marital status } \\
\text {-single or } \\
\text { divorced }\end{array}$ & Minutes per week & -0.52 & 3.05 & -6.49 & 5.45 & 0.864 \\
\hline $\begin{array}{l}\text { Married or } \\
\text { widowed }\end{array}$ & Steps per week & -11216.56 & 11224.05 & -33215.31 & 10782.18 & 0.318 \\
\hline $\begin{array}{l}\text { Educational } \\
\text { level - college } \\
\text { or higher }\end{array}$ & Minutes per week & -200.33 & 52.66 & -303.54 & -97.12 & 0.000 \\
\hline $\begin{array}{l}\text { High school or } \\
\text { lower }\end{array}$ & Minutes per week & 8321.68 & 6453.39 & -4326.74 & 20970.09 & 0.197 \\
\hline $\begin{array}{l}\text { Medical } \\
\text { conditions not } \\
\text { present }\end{array}$ & Steps per week & -98855.34 & 13626.83 & -36593.44 & 16822.76 & 0.468 \\
\hline Present & Minutes per week & 150.10 & 74 & -55.99 & 234.08 & 0.229 \\
\hline
\end{tabular}

Field notes: Patients of Clinica Amistad, where this study was conducted, eagerly volunteered to participate in the study. Field notes analyses indicated that participants expressed satisfaction with the study materials provided. Some participants were very enthusiastic about the use of 
pedometers, and expressed they had never used a pedometer before. Participants commented that pedometer use reminded them of the need to exercise. Similarly, participants stated that the PA logs were easy to complete. Participants also expressed positive comments regarding the weekly PA intervention demonstration. One of the participants stated "I really like walking in a group. Being involved in this (study) is a good thing, it is good for our health.” Another participant described the intervention as "it is really easy to follow; when I see the pedometer in the morning, it reminds me that I need to walk for that day.” While the researcher originally arranged for the weekly meetings to take place at a community center meeting room near the clinic, one of the groups preferred to hold the meetings and PA intervention in a park near their places of residence. Participants from the park group stated that it was easier for them to meet at the park rather than the community center. Participants reported that a sense of pride and wellbeing came with participating in this pilot study. Some of the participants expressed surprise at the ease of being able to walk to improve their health. Participants were under the impression that they were going to have to join a health club to be able to participate in PA. Participants expressed interest in participating in future studies and stated that they would do so without the use of incentives. None of the participants expressed any negative comments regarding the intervention. Many of the participants were eager to enroll in future iterations of the Salud Paso por Paso study to continue their PA progress.

\section{Discussion}

The purpose of this pre-post, no control group pilot study was to examine the acceptability, feasibility and preliminary impact of a culturally-tailored PA intervention, Salud Paso por Paso, in a group of Hispanic adults living with T2DM. Findings indicate the PA intervention was acceptable to the priority population and participants had a significant increase 
in minutes of PA per week from pre-to post intervention. Effect sizes explain quantitatively relationships between variables (Schober et al., 2018). While effect sizes should be interpreted with caution as factors such as sample size may affect the reliability and precision of the estimate (Morris, 2019), the effect size for steps per week $(d=1.03)$ and minutes of PA per week $(d=1.62)$ show a potentially clinically significant impact of the intervention on PA engagement with participants.

Participants in this study had low acculturation scores, reflecting participants' strong identification with their identity as Hispanic. Evidence suggests that higher levels of acculturation are correlated to engaging in more leisure-time PA (Congello et al., 2020; Murillo et al., 2015). Conversely, low levels of acculturation have been associated with lower levels of self-rated health and lower use of medical services (Ellison et al., 2011). However, participants in this study, despite lower levels of acculturation, successfully increased their PA from pre to post intervention. The Salud Paso por Paso intervention was likely effective in this sample because the intervention was culturally-tailored, linguistically appropriate, and was developed in collaboration with community stakeholders. Of note, participants in this study had high selfefficacy for exercise scores at baseline. Previous research indicates that higher self-efficacy for exercise is associated with increased PA (Guntzviller et al., 2017).

Some participants did not overtly disclose the presence of health conditions, including T2DM, which may have occurred due to low health literacy levels. Hispanic immigrants and those with limited English proficiency may have lower levels of health literacy (Soto Mas et al., 2019). Some participants stated that their provider told them they had "el azucar alta (high sugar)," but these participants did not explicitly state they had T2DM. Another reason for participant reluctance to acknowledge they had T2DM may be due to cultural factors. 
Participants may have had perceived stigma of T2DM as a condition that was brought upon themselves or T2DM as a condition that will inevitably lead to serious sickness or death (SmithMiller et al., 2017).

It is notable that 19 participants (90\%) completed the intervention, even in light of the negative impact that the COVID-19 pandemic has had not only on conducting research, but on physical inactivity and society in general (Pinho et al., 2020). During the enrollment phase, the researcher had to reject additional potential participants as there was significant interest from Clinica Amistad patients to participate in the study. These potential participants learned about the study through word-of-mouth from enrolled participants. All of the study participants expressed interest in continuing with the intervention behaviors after the intervention ended. Some of the participants stated that they would be willing to enroll in a different study with a longer duration, as they perceived the 6-week intervention to end quickly. Results of the study were shared with the Clinica Amistad staff during meetings with providers (i.e physicians and nurse practitioners) and volunteers (i.e. nurse educators, triage nurses, and public health students). The focus of these meetings was to discuss the findings of the study and its implications for practice for Clinica Amistad patients.

Strengths of this research included its community-partnered approach and the fact that the researcher shared the cultural and linguistic background of participants. The fact that the researcher spent one year developing relationships with the Clinica Amistad community was felt to enhance the success of this study. While the findings of this study are promising with regard to increasing PA, there were limitations that must be considered. This was a small, non-controlled pilot study. A larger, prospective randomized study is needed to examine intervention efficacy. 
Another limitation is the potential negative impact the COVID-19 pandemic on intervention fidelity. The intervention study began just 2 weeks before an executive order from the governor of Arizona recommended social distancing and mask precautions (Arizona, Office of the Governor, 2020). While PA was an approved activity under this order and the researcher ensured all appropriate precautions (e.g. physical distancing) were maintained, many of the participants expressed concern about engaging in PA. Another limitation was the lack of completion of the 7-Day Physical Activity Recall (7-Day PAR) questionnaire (Sallis et al., 1988) by any of the participants. The researcher attempted to collect PA recall data pre- postintervention using the 7-Day PAR in addition to the pedometer-measured steps and participantreported minutes of PA. The 7-Day PAR estimates an individual's time spent in PA, strength, and flexibility activities (Hayden-Wade et al., 2003). None of the participants fully completed the pre- and post-intervention 7-Day PAR. Some of the participants recorded some data, but not enough to warrant analysis or inclusion of results. The researcher provided instructions on completion and tried to clarify any questions, but participants stated that they found the 7-Day PAR “confusing." Participants expressed that they had difficulty recalling PA performed each day of the previous week. This difficulty was markedly apparent during the completion of the pre-intervention 7-Day PAR, as participants did not have completed daily PA logs to enhance their recall. Perhaps utilizing a PA recall instrument that has a shorter recall window could provide more robust data. Results from a study comparing a shorter recall period of 24 hours instead of 7 days showed improved quality of PA recall in participants (Novak et al., 2020). Future iterations of Salud Paso por Paso should incorporate questionnaires with a shorter PA recall period to capture more data with Hispanic adults with T2DM. 
The need for effective interventions that promote PA in Hispanic adults, and the general population of individuals living with diabetes, is considerable. In particular, the Hispanic population is disproportionately affected by negative T2DM health outcomes. As the Salud Paso por Paso intervention was being conducted, many participants expressed interest in learning more about other behaviors that can enhance T2DM outcomes, such as nutrition. While the focus of this intervention was engaging participants in PA, future interventions could be more comprehensive, and the duration of the intervention could be longer. Other strategies to enhance engagement in PA could be added to the intervention, such as doing PA during a lunch break on a workday (Yoko et al., 2021). Significant results for PA outcomes, specifically number of steps and minutes, support a prospective, randomized, controlled study to examine the efficacy of Salud Paso por Paso among Hispanic adults living with T2DM. Moreover, future research should include measures to examine the ability for long-term sustainment of PA improvements. Since this was a feasibility and acceptability study, results from this study could inform the planning and completion of a randomized controlled trial for future research. A randomized control trial should not only build on the results of this study, but deliver an enhanced intervention by extending the duration of the study and providing additional components such as strength training and nutritional education tailored to Hispanic adults with T2DM. Developing effective interventions that can ameliorate the deleterious effects of T2DM in Hispanic adults will lead to a healthier community. 


\section{References}

Aguayo-Mazzucato, C., Diague, P., Hernandez, S., Rosas, S., Kostic, A., \& Caballero, A. E. (2019). Understanding the growing epidemic of type 2 diabetes in the Hispanic population living in the United States. Diabetes Metabolism Research and Reviews, 35(2), e3097. https://doi.org/10.1002/dmrr.3097

American Diabetes Association. (2017). Standards of medical care in diabetes - 2017. http://professional.diabetes.org/sites/professional.diabetes.org/files/ media/dc_40_s1_final.pdf

American Heart Association. (2014). American Heart Association recommendations for physical activity in adults. http://www.heart.org/HEARTORG/GettingHealthy/ PhysicalActivity/FitnessBasics/American-Heart-Association-Recommendations-forPhysical-Activity-in-Adults_UCM_307976_Article.jsp

Arizona, Office of the Governor. (2020, March 30). "Stay home, stay healthy, stay connected" [Press release]. https://azgovernor.gov/governor/news/2020/03/stayhome-stay-healthy-stay-connected

Arredondo, E. M., Sotres-Alvarez, D., Stoutenberg, M., Davis, S. M., Crespo, N. C., Carnethon, M. R., Castañeda, S. F., Isasi, C. R., Espinoza, R. A., Daviglus, M. L., Perez, L. G., \& Evenson, K. R. (2016). Physical activity levels in U.S. Latino/Hispanic adults: Results from the Hispanic Community Health Study/Study of Latinos. American Journal of Preventive Medicine, 50(4), 500-508. https://doi.org/10.1016/j.amepre.2015.08.029

Bandura, A. (1989). Human agency in social cognitive theory. American Psychologist, 44(9), 1175-1184. https://doi.org/10.1037/0003-066X.44.9.1175 
Bandura, A. (2004). Health promotion by social cognitive means. Health Education \& Behavior, 31(2), 143-164. https://doi.org/10.1177/1090198104263660

Branch, M., German, C., Bertoni, A., \& Yeboah, J. (2019). Incremental risk of cardiovascular disease and/or chronic kidney disease for future ASCVD and mortality in patients with type 2 diabetes mellitus: ACCORD trial. Journal of Diabetes and its Complications, 33(7), 468-472. https://doi.org/10.1016/j.jdiacomp.2019.04.004

Centers for Disease Control and Prevention. (2019a). Diabetes basics: Type 2 diabetes. https://www.cdc.gov/diabetes/basics/type2.html

Centers for Disease Control and Prevention. (2019b). Living with diabetes - Prevent complications. https://www.cdc.gov/diabetes/managing/problems.html

Centers for Disease Control and Prevention. (2020a). Diabetes basics: Prediabetes - your chance to prevent type 2 diabetes. https://www.cdc.gov/diabetes/basics/prediabetes.html

Centers for Disease Control and Prevention. (2020b). Diabetes basics: Diabetes risk factors. https://www.cdc.gov/diabetes/basics/risk-factors.html

Centers for Disease Control and Prevention. (2021, April 7). Hispanic/Latino Americans and type 2 diabetes. https://www.cdc.gov/diabetes/library/features/hispanic-diabetes.html Congello, N. C., Koniak-Griffin, D., Brecht, M. L., Hays, R. D., Heilemann, M. V., \& Nyamathi, A. M. (2020). Associations of partner support and acculturation with physical activity in Mexican American women. Hispanic Health Care International, 18(2), 98-104. https://doi.org/10.1177/1540415319886797 
Duvivier, B. M., Schaper, N. C., Hesselink, M. K., van Kan, L., Stienen, N., Winkens, B., Koster, A., \& Savelberg, H. H. (2017). Breaking sitting with light activities vs structured exercise: A randomised crossover study demonstrating benefits for glycaemic control and insulin sensitivity in type 2 diabetes. Diabetologia, 60(3), 490-498.

https://doi.org/10.1007/s00125-016-4161-7

Ellison, J., Jandorf, L., \& Duhamel, K. (2011). Assessment of the Short Acculturation Scale for Hispanics (SASH) among low-income, immigrant Hispanics. Journal of Cancer Education, 26(3), 478-483. https://doi.org/10.1007/s13187-011-0233-z

Glantz, N. M., Duncan, I., Ahmed, T., Fan, L., Reed, B. L., Kalirai, S., \& Kerr, D. (2019). Racial and ethnic disparities in the burden and cost of diabetes for US Medicare beneficiaries. Health Equity, 3(1), 211-218. https://doi.org/10.1089/heq.2019.0004

Guntzviller, L. M., King, A. J., Jensen, J. D., \& Davis, L. A. (2017). Self-efficacy, health literacy, and nutrition and exercise behaviors in a low-income, Hispanic population. Journal of Immigrant and Minority Health, 19(2), 489-493. https://doi.org/10.1007/s10903-016-0384-4

Hayden-Wade, H. A., Coleman, K. J., Sallis, J. F., \& Armstrong, C. (2003). Validation of the telephone and in-person interview versions of the 7-day PAR. Medicine and Science in Sports and Exercise, 35, 801-809

Loya, J. C. (2018). Systematic review of physical activity interventions in Hispanic adults. Hispanic Health Care International, 16(4), 174-188. https://doi.org/10.1177/1540415318809427 
Marin, G., Sabogal, F., Marin, B. V., Otero-Sabogal, R., \& Perez-Stable, E. J. (1987).

Development of a short acculturation scale for Hispanics. Hispanic Journal of Behavioral Sciences, 9(2), 183-205.

McCurley, J. L., Gutierrez, A. P., \& Gallo, L. C. (2017). Diabetes prevention in U.S. Hispanic adults: A systematic review of culturally tailored interventions. American Journal of Preventive Medicine, 52(4), 519-529. https://doi.org/10.1016/j.amepre.2016.10.028

Morris P. H. (2020). Misunderstandings and omissions in textbook accounts of effect sizes. British Journal of Psychology, 111(2), 395-410. https://doi.org/10.1111/bjop.12401

Murillo, R., Albrecht, S. S., Daviglus, M. L., \& Kershaw, K. N. (2015). The role of physical activity and sedentary behaviors in explaining the association between acculturation and obesity among Mexican-American adults. American Journal of Health Promotion, 30(1), 50-57. https://doi.org/10.4278/ajhp.140128-QUAN-49

Myers, J., Kokkinos, P., \& Nyelin, E. (2019). Physical activity, cardiorespiratory fitness, and the metabolic syndrome. Nutrients, 11(7), 1652. https://doi.org/10.3390/nu11071652

Noe-Bustamante, L., Lopez, M. H., \& Krogstad, J. M. (2020, July 7). U.S. Hispanic population surpassed 60 million in 2019, but growth has slowed. Pew Research Center. https://www.pewresearch.org/fact-tank/2020/07/07/u-s-hispanic-population-surpassed60-million-in-2019-but-growth-has-slowed/

Novak, B., Holler, P., Jaunig, J., Ruf, W., van Poppel, M., \& Sattler, M. C. (2020). Do we have to reduce the recall period? Validity of a daily physical activity questionnaire (PAQ24) in young active adults. BMC Public Health, 20(1), 72. https://doi.org/10.1186/s12889-020-8165-3 
Office of Minority Health. (n.d.). Culturally competent nursing care: A cornerstone of caring. https://thinkculturalhealth.hhs.gov/education/nurses

Pinho, C. S., Caria, A., Aras Júnior, R., \& Pitanga, F. (2020). The effects of the COVID-19 pandemic on levels of physical fitness. Revista da Associacao Medica Brasileira, 66(Suppl 2), 34-37. https://doi.org/10.1590/1806-9282.66.S2.34

Punthakee, Z., Goldenberg, R., \& Katz, P. (2018). Definition, classification and diagnosis of diabetes, prediabetes and metabolic syndrome. Canadian Journal of Diabetes, $42 \mathrm{Suppl}$ 1, S10-S15. https://doi.org/10.1016/j.jcjd.2017.10.003

Sallis, J. F., Haskell, W. L., Wood, P. D., Fortmann, S. P., Rogers, T., Blair, S. N., \& Paffenbarger, R. (1985). Physical activity assessment methodology in the Five City Project. American Journal of Epidemiology, 121, 91-106.

Sallis, J. F., Pinski, R. B., Grossman, R. M., Patterson, T. L., \& Nader, P. R. (1988). The development of self-efficacy scales for health-related diet and exercise behaviors. Health Education Research, 3(3), 283-292.

Schober, P., Bossers, S. M., \& Schwarte, L. A. (2018). Statistical significance versus clinical importance of observed effect sizes: What do $p$ values and confidence intervals really represent? Anesthesia and Analgesia, 126(3), 1068-1072. https://doi.org/10.1213/ANE.0000000000002798

Smith-Miller, C. A., Berry, D. C., \& Miller, C. T. (2017). Diabetes affects everything: Type 2 diabetes self-management among Spanish-speaking Hispanic immigrants. Research in Nursing \& Health, 40(6), 541-554. https://doi.org/10.1002/nur.21817 
Soto Mas, F., Jacobson, H. E., \& Olivárez, A. (2017). Adult education and the health literacy of Hispanic immigrants in the United States. Journal of Latinos and Education, 16(4), 314322. https://doi.org/10.1080/15348431.2016.1247707

Tucker, J. M., Welk, G. J., \& Beyler, N. K. (2011). Physical activity in U.S.: Adults compliance with the Physical Activity Guidelines for Americans. American Journal of Preventive Medicine, 40(4), 454-461. https://doi.org/10.1016/j.amepre.2010.12.016

U.S. Census Bureau. (2018, October 9). Hispanic population to reach 111 million by 2060. Census.gov. https://www.census.gov/library/visualizations/2018/comm/hispanicprojected-pop.html

U.S. Department of Health and Human Services. (2021). Executive summary: Physical activity guidelines for Americans ( $2^{\text {nd }}$ ed.). https://health.gov/sites/default/files/2019-10/ PAG_ExecutiveSummary.pdf

Yoko, N., Hiroshi, Y., \& Ying, J. (2021). Type and timing of exercise during lunch breaks for suppressing postprandial increases in blood glucose levels in workers. Journal of Occupational Health, 63(1), e12199. https://doi.org/10.1002/1348-9585.12199 


\section{CHAPTER 5}

\section{CONCLUSION}

This pilot study demonstrated the feasibility, acceptability and potential effectiveness of a novel, culturally-tailored PA intervention named Salud Paso por Paso. The novel intervention was developed for sedentary Hispanic adults living with T2DM. Most of the individuals (71\%) who participated in the Salud Paso por Paso intervention demonstrated the ability to increase PA gradually over 6 weeks. The results of this study were significant with regard to main outcome measures of steps and minutes of PA per week. Participants received pedometers and educational materials as a strategy to promote behavioral PA changes. One group chose to meet at a park, and the other group chose to meet at the community center. Both groups had similar results. The park group chose the park as it was conveniently located near their place of residence.

Participants were able to walk to the park from their homes. The community center group chose the community center setting as it was located near the clinic. Many of these participants had to take public transportation to attend the intervention sessions, and a city bus transit center is located adjacent to the clinic.

This study has demonstrated that sedentary Hispanic adults with T2DM are receptive to community-partnered approach to increase PA. It appears that the fact that the study was delivered in a setting where participants were comfortable (i.e. Clinica Amistad) enhanced engagement in the study. The Salud Paso por Paso intervention was developed in partnership with a trusted source of health care in the community. Clinica Amistad has been serving patients since 2004, and there are many long-term patients that rely heavily on Clinica Amistad to meet their health care needs. Recruiting participants from a trusted venue, such as Clinica Amistad was thought to enhance the feasibility and acceptability of the intervention. All participants 
received clearance from a Clinica Amistad health care provider before participating in the intervention. Furthermore, the researcher built trust with clinic patients by volunteering as a triage nurse for a prolonged time prior to recruiting participants.

This trust paid dividends as the majority of intervention activities for Salud Paso por Paso occurred during the height of the COVID-19 pandemic. The intervention was conducted from the beginning of March 2020 to the end of May 2020. This was a period of uncertainty as there was no clear indication of how widespread COVID-19 disease was or a clear plan on how to prevent the spread of infection of SARS-CoV2 (Islam et al., 2020). As more information emerged about the transmission of SARS-CoV2, there were measures such as state-level executive orders in Arizona limiting group gatherings and taking appropriate precautions such as masking or maintaining physical distance. To this end, one of the Salud Paso por Paso groups decided to meet in a park, rather than the community center, as this was more convenient for them. During the first two weekly meetings, there were no group size or masking restrictions in place. Prior to meeting during the third week, the governor of Arizona implemented executive orders that required physical distancing and masking. The research had to adapt the meetings to ensure physical distancing and required masking was maintained by all participants. Some participants expressed concerns about engaging in PA in a group setting. The researcher explained to participants that the executive order permitted engaging in PA as long as physical distancing and masking requirements were maintained. The trust that had been built by the researcher, and Clinica Amistad, was felt to enhance retention in the study and demonstrates the strength of the community-partnered approach (Ceasar et al., 2019; Ceasar et al., 2017). Even in the midst of the COVID-19 pandemic, 90\% of participants were retained and the Salud Paso por Paso intervention yielded significant PA behavior change. 
More research is needed to examine the efficacy and long-term sustainability of behavior changes resulting from the Salud Paso por Paso intervention. Future studies should consider a prospective, wait-listed randomized controlled trial. To better understand the impact of the intervention on outcomes, future research design will require the implementation of a fully powered study that addresses not only PA, but other factors that affect health outcomes in T2DM. T2DM is a chronic condition requiring various behavioral changes to manage; these include nutritional and PA behavior changes, medication management, and access to care. Additionally, barriers to care in Hispanic adults with T2DM may include inability to understand T2DM educational materials, having to work, lack of funds/time to manage T2DM, lack of providers, and lack of programs for diabetes self-management education (DSME) (Titus \& Kataoka-Yahiro, 2021). Future iterations of this study should include a comprehensive intervention that addresses the various factors that can affect health outcomes in T2DM. The intervention should include elements such as DSME, nutritional education, strength training in addition to PA education. One aspect of this study that should be used in a prospective randomized controlled trial is continuing to culturally tailor the intervention to better serve prospective participants.

The focus of this study was to elucidate the impact of a culturally-tailored PA intervention. Participants from this study expressed a desire to have a more comprehensive experience where they also received educational material on nutritional aspects to manage T2DM. Future research could incorporate additional elements such as strength training and nutritional components as part of the intervention with appropriate associated outcome measures (e.g. recording nutritional intake, BMI). 
Participants responded positively to the researcher being a trusted provider of health services at the recruitment site. Participants appreciated the intervention being delivered in Spanish, as they stated they felt more comfortable being able to speak and read in their primary language. Participants also enthusiastically endorsed the use of pedometers to record PA. Many of the participants had not used a pedometer before, and they found the visualization their daily steps as a helpful tool to record their progress and to motivate them to continue engaging in PA. With regard to clinical practice, the findings from this study also have implications. Salud Paso por Paso study showed that Hispanic adults with T2DM are willing to engage in behavior change to improve health outcomes. The benefits of engaging in PA regularly for individuals with T2DM are well-established (Amanat et al., 2020; Kirwan et al., 2017). The results of the Salud Paso por Paso were shared with the Clinica Amistad staff. Meetings were conducted during clinic hours with providers (i.e. physicians and nurse practitioners) and volunteers (i.e. nurse educators, triage nurses, and public health students) to discuss the findings and its implications for practice. Clinic audiences agreed that this intervention had a significant impact on the health of participants. Furthermore, clinic staff expressed a willingness to continue encouraging clinic patients to engage in PA. Clinic staffed mentioned that some of the participants of this study had improved their attendance to clinic appointments. While this increase many not have been directly associated with the study, it was a welcome change as these participants had been inconsistent with attending their appointments previous to completion of this study. The researcher explained to the clinic audience that results from this study will be used to support grant applications to fund a fully powered study where more participants can enroll and potentially benefit. 
The researcher has continued to volunteer at the clinic as a triage nurse. After the completion of this intervention, the clinic secured funding from a donor that would like to implement an intervention that incorporates cooking and PA to benefit individuals with prediabetes. The researcher has volunteered to assist with the implementation of this initiative, which strives to prevent progression from pre-diabetes to T2DM.

In conclusion, the Salud Paso por Paso intervention has demonstrated that it is feasible to engage Hispanic adults with T2DM in PA and that the intervention increased PA over a 6-week period. To eliminate health disparities, culturally-tailored interventions, such as Salud Paso por Paso, are needed. This intervention showed that despite significant challenges, such as the COVID-19 pandemic, individuals with T2DM are still eager to participate in research that will possibly benefit them. As the incidence of T2DM in Hispanic adults continues to increase, interventions such as Salud Paso por Paso may be effective strategies to ameliorate the potential negative impact of T2DM in this population. 


\section{References}

Allen, N. A., Colicchio, V. D., Litchman, M. L., Gibson, B., Villalta, J., \& Sanchez-Birkhead, A. C. (2019). Hispanic community-engaged research: Community partners as our teachers to improve diabetes self-management. Hispanic Health Care International, 17(3), 125-132. https://doi.org/10.1177/1540415319843229

Amanat, S., Ghahri, S., Dianatinasab, A., Fararouei, M., \& Dianatinasab, M. (2020). Exercise and type 2 diabetes. Advances in Experimental Medicine and Biology, 1228, 91-105. https://doi.org/10.1007/978-981-15-1792-1_6

Ceasar, J. N., Claudel, S. E., Andrews, M. R., Tamura, K., Mitchell, V., Brooks, A. T., Dodge, T., El-Toukhy, S., Farmer, N., Middleton, K., Sabado-Liwag, M., Troncoso, M., Wallen, G. R., \& Powell-Wiley, T. M. (2019). Community engagement in the development of an mhealth-enabled physical activity and cardiovascular health intervention (Step It Up): Pilot focus group study. JMIR Formative Research, 3(1), e10944. https://doi.org/10.2196/10944

Islam, M. S., Rahman, K. M., Sun, Y., Qureshi, M. O., Abdi, I., Chughtai, A. A., \& Seale, H. (2020). Current knowledge of COVID-19 and infection prevention and control strategies in healthcare settings: A global analysis. Infection Control and Hospital Epidemiology, 41(10), 1196-1206. https://doi.org/10.1017/ice.2020.237

Kirwan, J. P., Sacks, J., \& Nieuwoudt, S. (2017). The essential role of exercise in the management of type 2 diabetes. Cleveland Clinic Journal of Medicine, 84(7 Suppl 1), S15-S21. https://doi.org/10.3949/ccjm.84.s1.03 
Larsen, B., Dunsiger, S. I., Pekmezi, D., Linke, S., Hartman, S. J., \& Marcus, B. H. (2021). Psychosocial mediators of physical activity change in a web-based intervention for Latinas. Health Psychology, 40(1), 21-29. https://doi.org/10.1037/hea0001041

Mansyur, C. L., Rustveld, L. O., Nash, S. G., \& Jibaja-Weiss, M. L. (2016). Hispanic acculturation and gender differences in support and self-efficacy for managing diabetes. The Diabetes Educator, 42(3), 315-324.

https://doi.org/10.1177/0145721716640905

Mendoza-Vasconez, A. S., Arredondo, E. M., Larsen, B., Crespo, N., Hurst, S., \& Marcus, B. H. (2021). Lapse, relapse, and recovery in physical activity interventions for Latinas: A survival analysis. International Journal of Behavioral Medicine. Advance online publication. https://doi.org/10.1007/s12529-020-09943-Z

Titus, S. K., \& Kataoka-Yahiro, M. (2021). Barriers to access to care in Hispanics with type 2 diabetes: A systematic review. Hispanic Health Care International, 19(2), 118-130. https://doi.org/10.1177/1540415320956389 


\section{COMPREHENSIVE REFERENCE LIST}

Aguayo-Mazzucato, C., Diague, P., Hernandez, S., Rosas, S., Kostic, A., \& Caballero, A. E. (2019). Understanding the growing epidemic of type 2 diabetes in the Hispanic population living in the United States. Diabetes Metabolism Research and Reviews, 35(2), e3097. https://doi.org/10.1002/dmrr.3097

Allen, N. A., Colicchio, V. D., Litchman, M. L., Gibson, B., Villalta, J., \& Sanchez-Birkhead, A. C. (2019). Hispanic community-engaged research: Community partners as our teachers to improve diabetes self-management. Hispanic Health Care International, 17(3), 125-132. https://doi.org/10.1177/1540415319843229

Amanat, S., Ghahri, S., Dianatinasab, A., Fararouei, M., \& Dianatinasab, M. (2020). Exercise and type 2 diabetes. Advances in Experimental Medicine and Biology, 1228, 91-105. https://doi.org/10.1007/978-981-15-1792-1_6

American Diabetes Association. (2017). Standards of medical care in diabetes - 2017. http://professional.diabetes.org/sites/professional.diabetes.org/files/ media/dc_40_s1_final.pdf

American Heart Association. (2014). American Heart Association recommendations for physical activity in adults. http://www.heart.org/HEARTORG/GettingHealthy/ PhysicalActivity/FitnessBasics/American-Heart-Association-Recommendations-forPhysical-Activity-in-Adults_UCM_307976_Article.jsp

Arizona, Office of the Governor. (2020, March 30). "Stay home, stay healthy, stay connected" [Press release]. https://azgovernor.gov/governor/news/2020/03/stayhome-stay-healthy-stay-connected 
Arredondo, E. M., Elder, J. P., Haughton, J., Slymen, D. J., Sallis, J. F., Perez, L. G., Serrano, N., Parra, M. T., Valdivia, R., \& Ayala, G. X. (2017). Fe en Acción: Promoting physical activity among churchgoing Latinas. American Journal of Public Health, 107(7), 11091115. https://doi.org/10.2105/AJPH.2017.303785

Arredondo, E. M., Sotres-Alvarez, D., Stoutenberg, M., Davis, S. M., Crespo, N. C., Carnethon, M. R., Castañeda, S. F., Isasi, C. R., Espinoza, R. A., Daviglus, M. L., Perez, L. G., \& Evenson, K. R. (2016). Physical activity levels in U.S. Latino/Hispanic adults: Results from the Hispanic Community Health Study/Study of Latinos. American Journal of Preventive Medicine, 50(4), 500-508. https://doi.org/10.1016/j.amepre.2015.08.029

Ayala, G. X., Vaz, L., Earp, J. A., Elder, J. P., \& Cherrington, A. (2010). Outcome effectiveness of the lay health advisor model among Latinos in the United States: An examination by role. Health Education Research, 25(5), 815-840. https://doi.org/10.1093/her/cyq035

Babamoto, K. S., Sey, K. A., Camilleri, A. J., Karlan, V. J., Catalasan, J., \& Morisky, D. E. (2009). Improving diabetes care and health measures among Hispanics using community health workers: Results from a randomized controlled trial. Health Education \& Behavior, 36(1), 113-126. https://doi.org/10.1177/1090198108325911

Bandura, A. (1989). Human agency in social cognitive theory. American Psychologist, 44(9), 1175-1184. https://doi.org/10.1037/0003-066X.44.9.1175

Bandura, A., \& Locke, E. A. (2003). Negative self-efficacy and goal effects revisited. Journal of Applied Psychology, 88(1), 87-99. https://doi.org/10.1037/0021-9010.88.1.87

Bandura, A. (2004). Health promotion by social cognitive means. Health Educucation \& Behavior, 31(2), 143-164. https://doi.org/10.1177/1090198104263660 
Bell, J. A., Hamer, M., Batty, G. D., Singh-Manoux, A., Sabia, S., \& Kivimaki, M. (2014). Combined effect of physical activity and leisure time sitting on long-term risk of incident obesity and metabolic risk factor clustering. Diabetologia, 57(10), 2048-2056. https://doi.org/10.1007/s00125-014-3323-8

Berry, N. S., McQuiston, C., Parrado, E. A., \& Olmos-Muñiz, J. C. (2013). Appendix G: Field notes guide. In B. A., Israel, E. Eng, A. Schulz, \& E. Parker (Eds.), Methods for community-based participatory research for health (2nd ed., 619-621). San Francisco, CA: Josey-Bass.

Branch, M., German, C., Bertoni, A., \& Yeboah, J. (2019). Incremental risk of cardiovascular disease and/or chronic kidney disease for future ASCVD and mortality in patients with type 2 diabetes mellitus: ACCORD trial. Journal of Diabetes and its Complications, 33(7), 468-472. https://doi.org/10.1016/j.jdiacomp.2019.04.004

Brown, A. (2014, February 26). The U.S. Hispanic population has increased sixfold since 1970. http://www.pewresearch.org/fact-tank/2014/02/26/the-u-s-hispanic-population-hasincreased-sixfold-since-1970/

Bullard, K. M., Cowie, C. C., Lessem, S. E., Saydah, S. H., Menke, A., Geiss, L. S., Orchard, T. J., Rolka, D. B., \& Imperatore, G. (2018). Prevalence of diagnosed diabetes in adults by diabetes type - United States, 2016. MMWR. Morbidity and Mortality Weekly Report, 67(12), 359-361. https://doi.org/10.15585/mmwr.mm6712a2

Caballero, A. E. (2005). Diabetes in the Hispanic or Latino population: Genes, environment, culture, and more. Current Diabetes Reports, 5(3), 217-225. https://doi.org/10.1007/s11892-005-0012-5 
Carter, W. M., Morse, W. C., Brock, R. W., \& Struempler, B. (2019). Improving physical activity and outdoor recreation in rural Alabama through community coalitions. Preventing Chronic Disease, 16, E116. https://doi.org/10.5888/pcd16.190062

Ceasar, J. N., Claudel, S. E., Andrews, M. R., Tamura, K., Mitchell, V., Brooks, A. T., Dodge, T., El-Toukhy, S., Farmer, N., Middleton, K., Sabado-Liwag, M., Troncoso, M., Wallen, G. R., \& Powell-Wiley, T. M. (2019). Community engagement in the development of an mhealth-enabled physical activity and cardiovascular health intervention (Step It Up): Pilot focus group study. JMIR Formative Research, 3(1), e10944. https://doi.org/10.2196/10944

Centers for Disease Control and Prevention. (2014). Physical activity - Facts about physical activity. http://www.cdc.gov/physicalactivity/data/facts/htm

Centers for Disease Control and Prevention. (2018). Overweight and obesity - Causes and consequences. http://www.cdc.gov/obesity/adult/causes/index.html

Centers for Disease Control and Prevention. (2019a, May 30). Diabetes basics: Type 2 diabetes. https://www.cdc.gov/diabetes/basics/type2.html

Centers for Disease Control and Prevention. (2019b, August 1). Living with diabetes - Prevent complications. https://www.cdc.gov/diabetes/managing/problems.html

Centers for Disease Control and Prevention. (2020, June 11). Diabetes basics: Prediabetes your chance to prevent type 2 diabetes.

https://www.cdc.gov/diabetes/basics/prediabetes.html

Centers for Disease Control and Prevention. (2021a, April 7). Hispanic/Latino Americans and type 2 diabetes. https://www.cdc.gov/diabetes/library/features/hispanic-diabetes.html 
Centers for Disease Control and Prevention. (2021b, April 23). Diabetes basics: Diabetes risk factors. https://www.cdc.gov/diabetes/basics/risk-factors.html

Cerin, E., Cain, K. L., Oyeyemi, A. L., Owen, N., Conway, T. L., Cochrane, T., VAN Dyck, D., Schipperijn, J., Mitáš, J., Toftager, M., Aguinaga-Ontoso, I., \& Sallis, J. F. (2016). Correlates of agreement between accelerometry and self-reported physical activity. Medicine and Science in Sports and Exercise, 48(6), 1075-1084. https://doi.org/10.1249/MSS.0000000000000870

Chen, P. G., Diaz, N., Lucas, G., \& Rosenthal, M. S. (2010). Dissemination of results in community-based participatory research. American Journal of Preventive Medicine, 39(4), 372-378. https://doi.org/10.1016/j.amepre.2010.05.021

Clínica Amistad. (n.d.). Quienes somos. http://www.clínicaamistad.org/ espanol/quienes-somos/

Coleman, K. J., Farrell, M. A., Rocha, D. A., Hayashi, T., Hernandez, M., Wolf, J., \& Lindsay, S. (2012). Readiness to be physically active and self-reported physical activity in lowincome Latinas, California WISEWOMAN, 2006-2007. Preventing Chronic Disease, 9, E87.

Congello, N. C., Koniak-Griffin, D., Brecht, M. L., Hays, R. D., Heilemann, M. V., \& Nyamathi, A. M. (2020). Associations of partner support and acculturation with physical activity in Mexican American women. Hispanic Health Care International, 18(2), 98-104. https://doi.org/10.1177/1540415319886797 
Conn, V. S., Phillips, L. J., Ruppar, T. M., \& Chase, J. A. (2012). Physical activity interventions with healthy minority adults: Meta-analysis of behavior and health outcomes. Journal of Health Care for the Poor and Underserved, 23(1), 59-80. https://doi.org/10.1353/hpu.2012.0032

Craig, C. L., Marshall, A. L., Sjöström, M., Bauman, A. E., Booth, M. L., Ainsworth, B. E., Pratt, M., Ekelund, U., Yngve, A., Sallis, J. F., \& Oja, P. (2003). International physical activity questionnaire: 12 -country reliability and validity. Medicine and Science in Sports and Exercise, 35(8), 1381-1395. https://doi.org/10.1249/01.MSS.0000078924.61453.FB

Duggan, C., Carosso, E., Mariscal, N., Islas, I., Ibarra, G., Holte, S., Copeland, W., Linde, S., \& Thompson, B. (2014). Diabetes prevention in Hispanics: Report from a randomized controlled trial. Preventing Chronic Disease, 11, E28. https://doi.org/10.5888/pcd11.130119

Duvivier, B. M., Schaper, N. C., Hesselink, M. K., van Kan, L., Stienen, N., Winkens, B., Koster, A., \& Savelberg, H. H. (2017). Breaking sitting with light activities vs structured exercise: A randomised crossover study demonstrating benefits for glycaemic control and insulin sensitivity in type 2 diabetes. Diabetologia, 60(3), 490-498. https://doi.org/10.1007/s00125-016-4161-7

Eakin, E. G., Bull, S. S., Riley, K., Reeves, M. M., Gutierrez, S., \& McLaughlin, P. (2007). Recruitment and retention of Latinos in a primary care-based physical activity and diet trial: The Resources for Health study. Health Education Resesearc, 22(3), 361-371. https://doi.org/10.1093/her/cyl095 
Eakin, E. G., Bull, S. S., Riley, K. M., Reeves, M. M., McLaughlin, P., \& Gutierrez, S. (2007). Resources for health: A primary-care-based diet and physical activity intervention targeting urban Latinos with multiple chronic conditions. Health Psychology, 26(4), 392400. https://doi.org/10.1037/0278-6133.26.4.392

Ellison, J., Jandorf, L., \& Duhamel, K. (2011). Assessment of the Short Acculturation Scale for Hispanics (SASH) among low-income, immigrant Hispanics. Journal of Cancer Education, 26(3), 478-483. https://doi.org/10.1007/s13187-011-0233-z

Eng, E., \& Blanchard, L. (2007). Action-oriented community diagnosis: A health education tool. International Quarterly of Community Health Education, 26(2), 141-158. https://doi.org/10.2190/8046-2641-7HN3-5637

Ennis, S. R., Ríos-Vargas, M., \& Albert, N. G. (2011). The Hispanic population: 2010. https://www.census.gov/prod/cen2010/briefs/c2010br-04.pdf

Gabrys, L., Sperzel, S., Bernhoerster, M., Banzer, W., \& Vogt, L. (2017). Real-time visual activity feedback for physical activity improvement in breast and colon cancer patients. Research in Sports Medicine, 25(1), 1-10.

https://doi.org/10.1080/15438627.2016.1258639

Garcia, L., Gold, E. B., Wang, L., Yang, X., Mao, M., \& Schwartz, A. V. (2012). The relation of acculturation to overweight, obesity, pre-diabetes and diabetes among U.S. MexicanAmerican women and men. Ethnicity \& Disease, 22(1), 58-64.

Glantz, N. M., Duncan, I., Ahmed, T., Fan, L., Reed, B. L., Kalirai, S., \& Kerr, D. (2019). Racial and ethnic disparities in the burden and cost of diabetes for US Medicare beneficiaries. Health Equity, 3(1), 211-218. https://doi.org/10.1089/heq.2019.0004 
Guntzviller, L. M., King, A. J., Jensen, J. D., \& Davis, L. A. (2017). Self-efficacy, health literacy, and nutrition and exercise behaviors in a low-income, Hispanic population. Journal of Immigrant and Minority Health, 19(2), 489-493. https://doi.org/10.1007/s10903-016-0384-4

Guttmacher, S., Kelly, P. J., \& Ruiz-Janecko, Y. (2010). Community-based health interventions: Principles and applications. San Francisco, CA: Jossey-Bass.

Harkness, A., Gattamorta, K. A., Estrada, Y., Jimenez, D., Kanamori, M., Prado, G., \& BeharZusman, V. (2020). Latinx health disparities research during COVID-19: Challenges and innovations. Annals of Behavioral Medicine, 54(8), 544-547. https://doi.org/10.1093/abm/kaaa054

Hawkins, M., Hosker, M., Marcus, B. H., Rosal, M. C., Braun, B., Stanek, E. J., 3rd, Markenson, G., \& Chasan-Taber, L. (2015). A pregnancy lifestyle intervention to prevent gestational diabetes risk factors in overweight Hispanic women: A feasibility randomized controlled trial. Diabetic Medicine, 32(1), 1080-115. https://doi.org/10.1111/dme.12601

Hayden-Wade, H. A., Coleman, K. J., Sallis, J. F., \& Armstrong, C. (2003). Validation of the telephone and in-person interview versions of the 7-day PAR. Medicine and Science in Sports and Exercise, 35(5), 801-809. https://doi.org/10.1249/01.mss.0000064941.43869.4e

Holbrook, E., Barreira, T., \& Kang, M. (2009). Validity and reliability of Omron pedometers for prescribed and self-paced walking. Medicine \& Science in Sports \& Exercise, 41(3), 670674. https://doi.org/10.1249/MSS.0b013e3181886095 
Hovell, M. F., Mulvihill, M. M., Buono, M. J., Liles, S., Schade, D. H., Washington, T. A., Manzano, R., \& Sallis, J. F. (2008). Culturally tailored aerobic exercise intervention for low-income Latinas. American Journal of Health Promotion, 22(3), 155-163. https://doi.org/10.4278/ajhp.22.3.155

Ickes, M. J., \& Sharma, M. (2012). A systematic review of physical activity interventions in Hispanic adults. Journal of Environmental and Public Health, 2012, 15. https://doi.org/10.1155/2012/156435

Islam, M. S., Rahman, K. M., Sun, Y., Qureshi, M. O., Abdi, I., Chughtai, A. A., \& Seale, H. (2020). Current knowledge of COVID-19 and infection prevention and control strategies in healthcare settings: A global analysis. Infection Control and Hospital Epidemiology, 41(10), 1196-1206. https://doi.org/10.1017/ice.2020.237

Israel, B. A., Coombe, C. M., Cheezum, R. R., Schulz, A. J., McGranaghan, R. J., Lichtenstein, R., Reyes, A. G., Clement, J., \& Burris, A. (2010). Community-based participatory research: A capacity-building approach for policy advocacy aimed at eliminating health disparities. American Journal of Public Health, 100(11), 2094-2102. https://doi.org/10.2105/AJPH.2009.170506

Joseph, R. P., Benitez, T. J., Ainsworth, B. E., Todd, M., \& Keller, C. (2018). Acculturation and physical activity among Latinas enrolled in a 12-month walking intervention. Western Journal of Nursing Research, 40(7), 942-960. https://doi.org/10.1177/0193945917692305

Kirwan, J. P., Sacks, J., \& Nieuwoudt, S. (2017). The essential role of exercise in the management of type 2 diabetes. Cleveland Clinic Journal of Medicine, 84(7 Suppl 1), S15-S21. https://doi.org/10.3949/ccjm.84.s1.03 
Keller, C., \& Fleury, J. (2006). Factors related to physical activity in Hispanic women. Journal of Cardiovascular Nursing, 21(2), 142-145.

Khare, M. M., Cursio, J. F., Locklin, C. A., Bates, N. J., \& Loo, R. K. (2014). Lifestyle intervention and cardiovascular disease risk reduction in low-income Hispanic immigrant women participating in the Illinois WISEWOMAN program. Journal of Community Health, 39(4), 737-746. https://doi.org/10.1007/s10900-014-9820-3

King, A. C., Bickmore, T. W., Campero, M. I., Pruitt, L. A., \& Yin, J. L. (2013). Employing virtual advisors in preventive care for underserved communities: Results from the COMPASS study. Journal of Health Communication, 18(12), 1449-1464. https://doi.org/10.1080/10810730.2013.798374

Kolt, G. S., Schofield, G. M., Kerse, N., Garrett, N., Ashton, T., \& Patel, A. (2012). Healthy Steps trial: Pedometer-based advice and physical activity for low-active older adults. The Annals of Family Medicine, 10(3), 206-212. https://doi.org/10.1370/afm.1345

Koniak-Griffin, D., Brecht, M. L., Takayanagi, S., Villegas, J., Melendrez, M., \& Balcazar, H. (2015). A community health worker-led lifestyle behavior intervention for Latina (Hispanic) women: Feasibility and outcomes of a randomized controlled trial. International Journal of Nursing Studies, 52(1), 75-87. https://doi.org/10.1016/j.jjurstu.2014.09.005

Kwon, S., Wang, M., \& Hawkins, M. (2016). Association between self-reported physical activity and obesity among White, Black, Hispanic, and Asian Americans: 2007 and 2009 BRFSS. Ethnicity \& Disease, 23(2), 129-135. 
Larsen, B., Dunsiger, S. I., Pekmezi, D., Linke, S., Hartman, S. J., \& Marcus, B. H. (2021). Psychosocial mediators of physical activity change in a web-based intervention for Latinas. Health Psychology, 40(1), 21-29. https://doi.org/10.1037/hea0001041

Loya, J. C. (2018). Systematic review of physical activity interventions in Hispanic adults. Hispanic Health Care International, 16(4), 174-188. https://doi.org/10.1177/1540415318809427

Mansyur, C. L., Rustveld, L. O., Nash, S. G., \& Jibaja-Weiss, M. L. (2016). Hispanic acculturation and gender differences in support and self-efficacy for managing diabetes. The Diabetes Educator, 42(3), 315-324.

https://doi.org/10.1177/0145721716640905

Marin, G., Sabogal, F., Marin, B. V., Otero-Sabogal, R., \& Perez-Stable, E. J. (1987).

Development of a short acculturation scale for Hispanics. Hispanic Journal of Behavioral Sciences, 9(2), 183-205.

Marcellus, L. (2004). Are we missing anything? Pursuing research on attrition. Canadian Journal of Nursing Research, 36(3), 82-98

Marcus, B. H., Dunsiger, S. I., Pekmezi, D. W., Larsen, B. A., Bock, B. C., Gans, K. M., Marquez, B., Morrow, K. M., \& Tilkemeier, P. (2013). The Seamos Saludables study: A randomized controlled physical activity trial of Latinas. American Journal of Preventive Medicine, 45(5), 598-605. https://doi.org/10.1016/j.amepre.2013.07.006 
Marcus, B. H., Hartman, S. J., Larsen, B. A., Pekmezi, D., Dunsiger, S. I., Linke, S., Marquez, B., Gans, K. M., Bock, B. C., Mendoza-Vasconez, A. S., Noble, M. L., \& Rojas, C. (2016). Pasos Hacia La Salud: A randomized controlled trial of an internet-delivered physical activity intervention for Latinas. The International Journal of Behavioral Nutrition and Physical Activity, 13, 62. https://doi.org/10.1186/s12966-016-0385-7

Marin, G., Sabogal, F., Marin, B. V., Otero-Sabogal, R., \& Perez-Stable, E. J. (1987). Development of a short acculturation scale for Hispanics. Hispanic Journal of Behavioral Sciences, 9(2), 183-205.

Marquez, D. X., Neighbors, C. J., \& Bustamante, E. E. (2010). Leisure time and occupational physical activity among racial or ethnic minorities. Medicine and Science in Sports and Exercise, 42(6), 1086-1093. https://doi.org/10.1249/MSS.0b013e3181c5ec05

Marshall, S. J., Nicaise, V., Ji, M., Huerta, C., Haubenstricker, J., Levy, S. S., Ainsworth, B., \& Elder, J. E. (2013). Using step cadence goals to increase moderate-to-vigorous-intensity physical activity. Medicine and Science in Sports and Exercise, 45(3), 592-602. https://doi.org/10.1249/MSS.0b013e318277a586

Martinez, S. M., Ainsworth, B. E., \& Elder, J. P. (2008). A review of physical activity measures used among US Latinos: Guidelines for developing culturally appropriate measures. Annals of Behavioral Medicine, 36(2), 195-207. https://doi.org/10.1007/s12160-008-9063-6

Martinez, S. M., Arredondo, E. M., \& Roesch, S. (2013). Physical activity promotion among churchgoing Latinas in San Diego, California: Does neighborhood cohesion matter? Journal of Health Psychology, 18(10), 1319-1329. https://doi.org/10.1177/1359105312462433 
McCurley, J. L., Gutierrez, A. P., \& Gallo, L. C. (2017). Diabetes prevention in U.S. Hispanic adults: A systematic review of culturally tailored interventions. American Journal of Preventive Medicine, 52(4), 519-529. https://doi.org/10.1016/j.amepre.2016.10.028

McEwen, M. M., Pasvogel, A., Murdaugh, C., \& Hepworth, J. (2017). Effects of a family-based diabetes intervention on behavioral and biological outcomes for Mexican American adults. Diabetes Educator, 43(3), 272-285. https://doi.org/10.1177/0145721717706031

Mendoza-Vasconez, A. S., Arredondo, E. M., Larsen, B., Crespo, N., Hurst, S., \& Marcus, B. H. (2021). Lapse, relapse, and recovery in physical activity interventions for Latinas: A survival analysis. International Journal of Behavioral Medicine. Advance online publication. https://doi.org/10.1007/s12529-020-09943-z

Mier, N., Medina, A. A., \& Ory, M. G. (2007). Mexican Americans with type 2 diabetes: Perspectives on definitions, motivators, and programs of physical activity. Preventing Chronic Disease, 4(2), A24.

Mier, N., Ory, M. G., \& Medina, A. A. (2010). Anatomy of culturally sensitive interventions promoting nutrition and exercise in Hispanics: A critical examination of existing literature. Health Promotion Practice, 11(4), 541-554. https://doi.org/10.1177/1524839908328991

Mitchell, D. C., Andrews, T., \& Schenker, M. B. (2015). Pasos Saludables: A pilot randomized intervention study to reduce obesity in an immigrant farmworker population. Journal of Occupational and Environmental Medicine, 57(10), 1039-1046.

https://doi.org/10.1097/jom.0000000000000535 
Moher, D., Liberati, A., Tetzlaff, J., Altman, D. G., \& and the PRISMA Group. (2009). Preferred reporting items for systematic reviews and meta-analyses: The PRISMA statement. Annals of Internal Medicine, 151(4), 264-269. https://doi.org/10.7326/0003-4819-151-4-200908180-00135

Moher, D., Schulz, K. F., \& Altman, D. G. (2001). The CONSORT statement: Revised recommendations for improving the quality of reports of parallel-group randomised trials. The Lancet, 357(9263), 1191-1194. https://doi.org/10.1016/S0140-6736(00)04337-3

Morris P. H. (2020). Misunderstandings and omissions in textbook accounts of effect sizes. British Journal of Psychology, 111(2), 395-410. https://doi.org/10.1111/bjop.12401

Murillo, R., Albrecht, S. S., Daviglus, M. L., \& Kershaw, K. N. (2015). The role of physical activity and sedentary behaviors in explaining the association between acculturation and obesity among Mexican-American adults. American Journal of Health Promotion, 30(1), 50-57. https://doi.org/10.4278/ajhp.140128-QUAN-49

Myers, J., Kokkinos, P., \& Nyelin, E. (2019). Physical activity, cardiorespiratory fitness, and the metabolic syndrome. Nutrients, 11(7), 1652. https://doi.org/10.3390/nu11071652

National Center on Health, Physical Activity and Disability. (2021). Diabetes: Cardiovascular training guidelines. https://www.nchpad.org/88/665/Diabetes

National Institute of Diabetes and Digestive and Kidney Diseases. (2017). Overweight and obesity statistics. https://www.niddk.nih.gov/health-information/healthstatistics/Pages/overweight-obesity-statistics.aspx\#c

Noe-Bustamante, L., Lopez, M. H., \& Krogstad, J. M. (2020, July 7). U.S. Hispanic population surpassed 60 million in 2019, but growth has slowed. Pew Research Center. https://www.pewresearch.org/fact-tank/2020/07/07/u-s-hispanic-population-surpassed60-million-in-2019-but-growth-has-slowed/ 
Novak, B., Holler, P., Jaunig, J., Ruf, W., van Poppel, M., \& Sattler, M. C. (2020). Do we have to reduce the recall period? Validity of a daily physical activity questionnaire (PAQ24) in young active adults. BMC Public Health, 20(1), 72.

https://doi.org/10.1186/s12889-020-8165-3

Office of Minority Health. (n.d.). Culturally competent nursing care: A cornerstone of caring. https://thinkculturalhealth.hhs.gov/education/nurses

Ogilvie, D., Foster, C. E., Rothnie, H., Cavill, N., Hamilton, V., Fitzsimons, C. F., Mutrie, N., \& Scottish Physical Activity Research Collaboration (2007). Interventions to promote walking: Systematic review. BMJ, 334(7605), 1204. https://doi.org/10.1136/bmj.39198.722720.BE

Pekmezi, D. W., Neighbors, C. J., Lee, C. S., Gans, K. M., Bock, B. C., Morrow, K. M., Marquez, B., Dunsiger, S., \& Marcus, B. H. (2009). A culturally adapted physical activity intervention for Latinas: A randomized controlled trial. American Journal of Preventive Medicine, 37(6), 495-500. https://doi.org/10.1016/j.amepre.2009.08.023

Perez, A., Fleury, J., \& Keller, C. (2010). Review of intervention studies promoting physical activity in Hispanic women. Western Journal of Nursing Research, 32(3), 341-362. https://doi.org/10.1177/0193945909351300

Pinho, C. S., Caria, A., Aras Júnior, R., \& Pitanga, F. (2020). The effects of the COVID-19 pandemic on levels of physical fitness. Revista da Associacao Medica Brasileira, 66(Suppl 2), 34-37. https://doi.org/10.1590/1806-9282.66.S2.34 
Poston, W. S., 2nd, Haddock, C. K., Olvera, N. E., Suminski, R. R., Reeves, R. S., Dunn, J. K., Hanis, C. L., \& Foreyt, J. P. (2001). Evaluation of a culturally appropriate intervention to increase physical activity. American Journal of Health Behavior, 25(4), 396-406. https://doi.org/10.5993/ajhb.25.4.5

Punthakee, Z., Goldenberg, R., \& Katz, P. (2018). Definition, classification and diagnosis of diabetes, prediabetes and metabolic syndrome. Canadian Journal of Diabetes, 42 Suppl 1, S10-S15. https://doi.org/10.1016/j.jcjd.2017.10.003

Rhodes, S. D., Foley, K. L., Zometa, C. S., \& Bloom, F. R. (2007). Lay health advisor interventions among Hispanics/Latinos: A qualitative systematic review. American Journal of Preventive Medicine, 33(5), 418-427. https://doi.org/10.1016/j.amepre.2007.07.023

Rosal, M. C., Ockene, I. S., Restrepo, A., White, M. J., Borg, A., Olendzki, B., Scavron, J., Candib, L., Welch, G., \& Reed, G. (2011). Randomized trial of a literacy-sensitive, culturally tailored diabetes self-management intervention for low-income Latinos: Latinos en Control. Diabetes Care, 34(4), 838-844. https://doi.org/10.2337/dc10-1981

Rosal, M. C., Olendzki, B., Reed, G. W., Gumieniak, O., Scavron, J., \& Ockene, I. (2005). Diabetes self-management among low-income Spanish-speaking patients: A pilot study. Annals of Behavioral Medicine, 29(3), 225-235. https://doi.org/10.1207/s15324796abm2903_9 
Rothschild, S. K., Martin, M. A., Swider, S. M., Tumialan Lynas, C. M., Janssen, I., Avery, E. F., \& Powell, L. H. (2014). Mexican American trial of community health workers: A randomized controlled trial of a community health worker intervention for Mexican Americans with type 2 diabetes mellitus. American Journal of Public Health, 104(8), 1540-1548. https://doi.org/10.2105/ajph.2013.301439

Sallis, J. F., Haskell, W. L., Wood , P. D., Fortmann, S. P., Rogers, T., Blair, S. N., \& Paffenbarger, R. (1985). Physical activity assessment methodology in the Five City Project. American Journal of Epidemiology, 121, 91-106.

Sallis, J. F., Pinski, R. B., Grossman, R. M., Patterson, T. L., \& Nader, P. R. (1988). The development of self-efficacy scales for healthrelated diet and exercise behaviors. Health Education Research, 3(3), 283-292.

Sangster, J., Church, J., Haas, M., Furber, S., \& Bauman, A. (2015). A comparison of the costeffectiveness of two pedometer-based telephone coaching programs for people with cardiac disease. Heart, Lung and Circulation, 24(5), 471-479. https://doi.org/10.1016/j.hlc.2015.01.008

Sarma, S., Devlin, R. A., Gilliland, J., Campbell, M. K., \& Zaric, G. S. (2015). The effect of leisure-time physical activity on obesity, diabetes, high BP and heart disease among Canadians: Evidence from 2000/2001 to 2005/2006. Health Economics, 24(12), 15311547. https://doi.org/10.1002/hec.3106

Schober, P., Bossers, S. M., \& Schwarte, L. A. (2018). Statistical significance versus clinical importance of observed effect sizes: What do $\mathrm{p}$ values and confidence intervals really represent? Anesthesia and Analgesia, 126(3), 1068-1072. https://doi.org/10.1213/ANE.0000000000002798 
Sharma, M. (2008). Physical activity interventions in Hispanic American girls and women. Obesity Reviews, 9(6), 560-571. https://doi.org/10.1111/j.1467-789X.2008.00501.x

Smith-Miller, C. A., Berry, D. C., \& Miller, C. T. (2017). Diabetes affects everything: Type 2 diabetes self-management among Spanish-speaking Hispanic immigrants. Research in Nursing \& Health, 40(6), 541-554. https://doi.org/10.1002/nur.21817

Soto Mas, F., Jacobson, H. E., \& Olivárez, A. (2017). Adult education and the health literacy of Hispanic immigrants in the United States. Journal of Latinos and Education, 16(4), 314322. https://doi.org/10.1080/15348431.2016.1247707

Stewart, A. L., King, A. C., \& Haskell, W. L. (1993). Endurance exercise and health-related quality of life in 50-65 year-old adults. The Gerontologist, 33(6), 782-789.

Taylor, P., Lopez, M. H., Martinez, J., \& Velasco, G. (2012, April 4). When labels don't fit: Hispanics and their views of identity. http://www.pewhispanic.org/2012/04/04/whenlabels-dont-fit-hispanics-and-their-views-of-identity/

Titus, S. K., \& Kataoka-Yahiro, M. (2021). Barriers to access to care in Hispanics with type 2 diabetes: A systematic review. Hispanic Health Care International, 19(2), 118-130. https://doi.org/10.1177/1540415320956389

Tucker, J. M., Welk, G. J., \& Beyler, N. K. (2011). Physical activity in U.S.: Adults compliance with the Physical Activity Guidelines for Americans. American Journal of Preventive Medicine, 40(4), 454-461. https://doi.org/10.1016/j.amepre.2010.12.016

Tudor-Locke, C., Williams, J. E., Reis, J. P., \& Pluto, D. (2002). Utility of pedometers for assessing physical activity. Sports Medicine, 32(12), 795-808. https://doi.org/10.2165/00007256-200232120-00004 
Turino, T. (2003). Nationalism and Latin American music: Selected case studies and theoretical considerations. Latin American Music Review / Revista de Musica Latinoamericana, 24(2), 169-209.

Turner, D. W. (2010). Qualitative interview design: A practical guide for novice investigators. The Qualitative Report, 15(3), 754-760.

U.S. Census Bureau. (2018, October 9). Hispanic population to reach 111 million by 2060. Census.gov. https://www.census.gov/library/visualizations/2018/comm/hispanicprojected-pop.html

U.S. Department of Health and Human Services. (2021). Executive summary: Physical activity guidelines for Americans ( $2^{\text {nd }}$ ed.). https://health.gov/sites/default/files/2019-10/ PAG_ExecutiveSummary.pdf

Vincent, D. (2009). Culturally tailored education to promote lifestyle change in Mexican Americans with type 2 diabetes. Journal of the American Academy of Nurse Practitioners, 21(9), 520-527. https://doi.org/10.1111/j.1745-7599.2009.00439.x

Ware Jr., J. E., Kosinski, M., \& Keller, S. D. (1996). A 12-Item Short-Form Health Survey: Construction of scales and preliminary tests of reliability and validity. Medical Care, 34(3), 220-233.

Wieland, M. L., Hanza, M., Weis, J. A., Meiers, S. J., Patten, C. A., Clark, M. M., Sloan, J. A., Novotny, P. J., Njeru, J. W., Abbenyi, A., Levine, J. A., Goodson, M., Capetillo, G., Osman, A., Hared, A., Nigon, J. A., \& Sia, I. G. (2018). Healthy immigrant families: Randomized controlled trial of a family-based nutrition and physical activity intervention. American Journal of Health Promotion, 32(2), 473-484. https://doi.org/10.1177/0890117117733342 
Whitley, H. P., Yong, E. V., \& Rasinen, C. (2015). Selecting an A1C point-of-care instrument. Diabetes Spectrum, 28(3), 201-208. https://doi.org/10.2337/diaspect.28.3.201

Wood, F. G. (2002). Ethnic differences in exercise among adults with diabetes. Western Journal of Nursing Research, 24(5), 502-515. https://doi.org/10.1177/019394502400446388

Yancey, A. K., Ortega, A. N., \& Kumanyika, S. K. (2006). Effective recruitment and retention of minority research participants. Annual Review of Public Health, 27(1), 1-28. https://doi.org/10.1146/annurev.publhealth.27.021405.102113

Yoko, N., Hiroshi, Y., \& Ying, J. (2021). Type and timing of exercise during lunch breaks for suppressing postprandial increases in blood glucose levels in workers. Journal of Occupational Health, 63(1), e12199. https://doi.org/10.1002/1348-9585.12199

Zuazagoitia, A., Montoya, I., Grandes, G., Arietaleanizbeascoa, M., Arce, V., Martinez, V, Sanchez, M., \& Sanchez, A. (2014). Reliability and validity of the 7-day Physical Activity Recall interview in a Spanish population. European Journal of Sport Science, 14(sup1), S361-S368. https://doi.org/10.1080/17461391.2012.705332 


\section{VITA}

Julio Cesar Loya is a native of Nogales, Sonora, Mexico; he spent his formative years in Tucson, Arizona. He is the proud son of the late Carmen V. Leon and Jorge L. Loya. He received a Bachelor of Science in Biology from the University of Arizona in 2002, an Associates of Applied Science in Nursing from Cochise College in 2004, and a Bachelor of Nursing from Northern Arizona University in 2010. He has 17 years of clinical experience in nursing, with over 11 years of experience in nursing leadership. He has been actively involved and has assumed leadership roles in various areas of nursing, including leading quality and patient safety initiatives, professional organizations, and volunteer activities. As a staff nurse and a nurse leader, he realized there is a gap in the care of Hispanic diabetic patients in the acute care setting and the community. After this realization, he decided to pursue a doctoral degree in 2014 at the University of Missouri-Columbia Sinclair School of Nursing. During his doctoral program, he was selected for the Sinclair Fellowship and as a NINR-funded T32 Health Science Pre-Doctoral Fellow. He currently resides in Marana, Arizona with his wife Krista who is pursuing a career in the legal field. Julio works as an associate director for the trauma progressive care unit at Banner University Medical Center Tucson Campus in Tucson, Arizona. 